\title{
CHARACTERIZATION OF THE REGULATION AND FUNCTION OF PHOSPHATIDYLINOSITOL 3,5-BISPHOSPHATE
}

\author{
by
}

\author{
Shannon Cheuk Ying Ho \\ M.Sc., Ryerson University, 2011 \\ H.B.Sc., University of Toronto Scarborough, 2009
}

\author{
A Dissertation \\ Presented to Ryerson University \\ in partial fulfillment of the \\ requirements for the degree of \\ Doctor of Philosophy \\ In the Program of \\ Molecular Science \\ Toronto, Ontario, Canada, 2016 \\ (C) Shannon Cheuk Ying Ho 2016
}




\section{AUTHOR'S DECLARATION}

I hereby declare that I am the sole author of this dissertation. This is a true copy of the dissertation, including any required final revisions, as accepted by my examiners.

I authorize Ryerson University to lend this dissertation to other institutions or individuals for the purpose of scholarly research.

I further authorize Ryerson University to reproduce this dissertation by photocopying or by other means, in total or in part, at the request of other institutions or individuals for the purpose of scholarly research.

I understand that my dissertation may be made electronically available to the public. 
Characterization of the Regulation and Function of Phosphatidylinositol 3,5-bisphosphate

Shannon Ho Cheuk Ying Ho

Doctor of Philosophy, 2016

Molecular Science, Ryerson University

\begin{abstract}
$\operatorname{PtdIns}(3,5) \mathrm{P}_{2}$ is a low abundance phosphoinositide that is involved in a variety of cellular processes. Most notably, PtdIns(3,5) $\mathrm{P}_{2}$ is known to regulate vacuolar/lysosomal morphology. Deficiency in PtdIns $(3,5) \mathrm{P}_{2}$ results in enlargement of the yeast vacuole and, an extensive vacuolation of the late endocytic compartments in higher eukaryotes $(1,2)$. In addition, $\operatorname{Ptd} \operatorname{Ins}(3,5) \mathrm{P}_{2}$ is also involved in cellular functions including membrane trafficking, autophagy, and vacuolar/lysosomal acidification. However, the current study provided evidence that shows that the vacuole/lysosomes of $\operatorname{Ptd} \operatorname{Ins}(3,5) \mathrm{P}_{2}$-deficient cells remain acidic. Hence, $\operatorname{PtdIns}(3,5) \mathrm{P}_{2}$ may not have a role in steady-state vacuolar/lysosomal acidification. $\operatorname{Ptd} \operatorname{Ins}(3,5) \mathrm{P}_{2}$ is synthesized by the Fab1 lipid kinase and degraded by the antagonistic Fig4 lipid phosphatase. Vac14, an adaptor protein, is known to interact with both Fab1 and Fig4 to form a complex on the vacuolar membrane. This study demonstrated that Vac14 is required to form a homodimer for its interaction with Fig4 and Fab1. In addition, formation of the homodimer is necessary for regulation of PtdIns(3,5) $\mathrm{P}_{2}$. Mutations in human Vac14 and Fig4 has been identified in patients with neurodegenerative diseases, such as amyotrophic lateral sclerosis and Charcot-Marie-Tooth Type 4J $(3,4)$. This study provides an important stepping stone in characterizing the regulatory mechanism and understanding the function of $\operatorname{PtdIns}(3,5) \mathrm{P}_{2}$.
\end{abstract}




\section{Acknowledgements}

First and foremost, I would like to thank my supervisor, Dr. Roberto Botelho. Rob has been a great mentor and provided tremendous support in both my academic and personal growth. His timely advice, meticulous scrutiny and encouragement were of vital importance to the completion of my thesis. Thank you for being so patient with me.

I also would like to thank my graduate committee, Dr. Debora Foster and Dr. Costin Antonescu. The encouragement and advice from Dr. Foster kept me motivated during the stressful times near the end of my study. The guidance and constructive feedback from Dr. Costin Antonescu throughout the years has been very helpful toward the development of my study.

Special thanks to Danielle Johnson for the technical training and advice that significantly impacted my thesis. In addition, I am especially thankful for the support from my friends in the Botelho lab and in the program. To Tamadher Alghamdi, Amra Saric, Grace Kim, Tracy Lackraj, Christopher Choy, Monica Dayam, Miriam de Jong, and many others, it has been great working with you guys and thanks for all the fun and joyful moments we shared together.

Finally, I would like to thank my parents and brothers for their unconditional support. 


\section{Table of Contents}

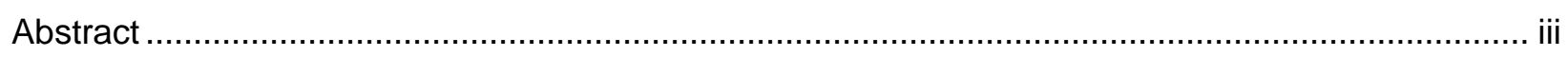

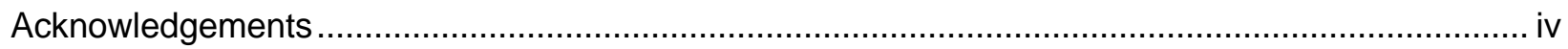

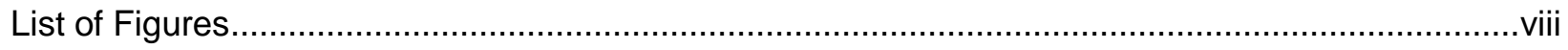

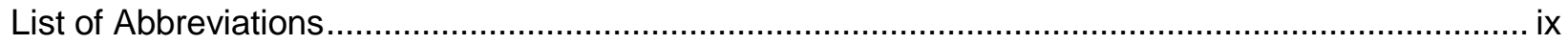

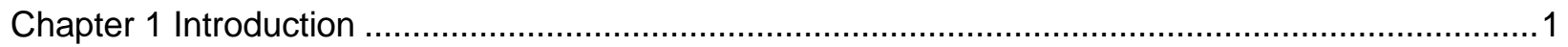

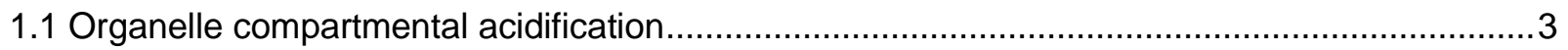

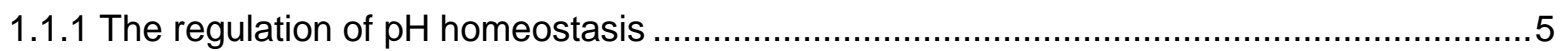

1.1.2 The importance of lysosomal/vacuolar acidification: Degradation .................................. 7

1.1.3 The importance of lysosomal/vacuolar acidification: Vesicular transport and fusion ..........8

1.1.4 The importance of lysosomal/vacuolar acidification: Storage of metabolites .....................9

1.1.5 The regulation and function of V-ATPase ............................................................. 10

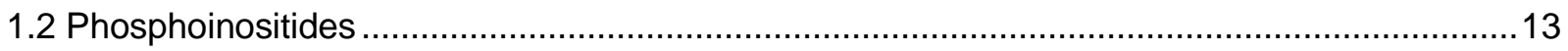

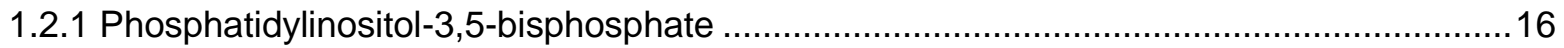

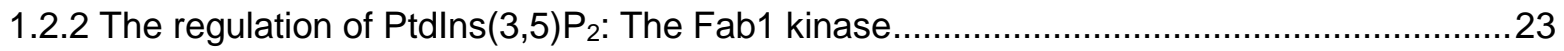

1.2.3 The regulation of PtdIns(3,5) $\mathrm{P}_{2}$ : The Fig4 phosphatase ..............................................25

1.2.4 The regulation of Ptdlns $(3,5) \mathrm{P}_{2}$ : The Vac14 adaptor................................................26

1.2.5 The regulation of Ptdlns $(3,5) \mathrm{P}_{2}$ : The Fab1 complex.....................................................27

1.2.6 The regulation of PtdIns(3,5) $\mathrm{P}_{2}$ : The Vac14 multimer .............................................. 32

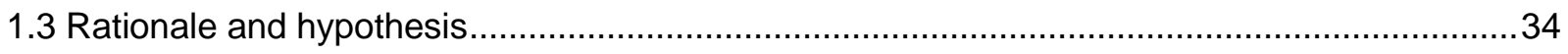

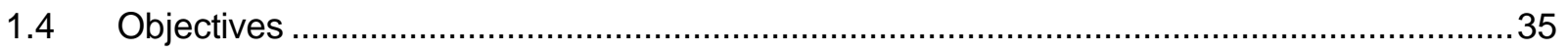

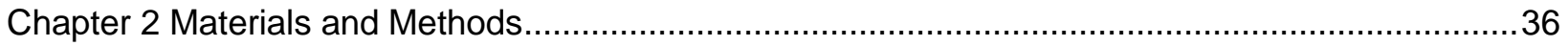

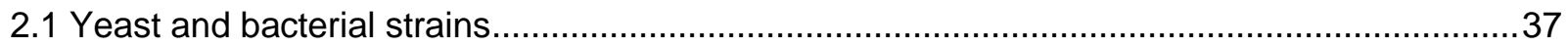

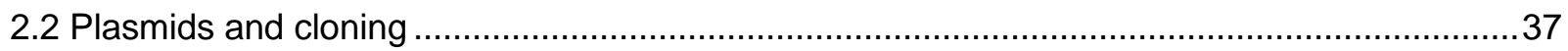

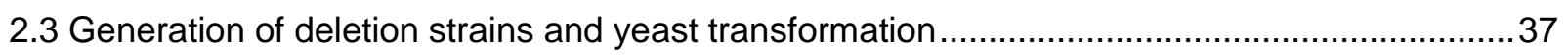

2.4 Recombinant protein expression and purification............................................................ 38

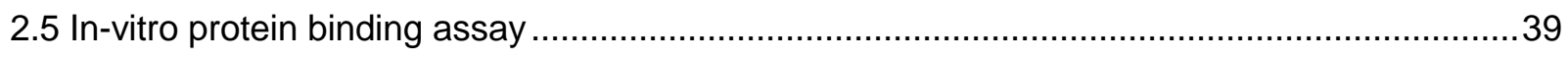

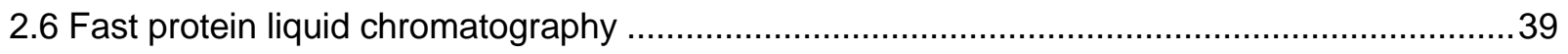

2.7 Differential velocity ultracentrifugation with glycerol gradient ................................................39

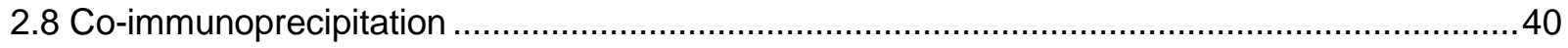

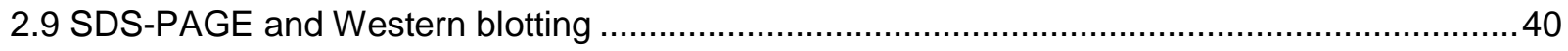

2.10 Vacuolar staining, hyperosmotic shock, and fluorescence microscopy ..............................41 


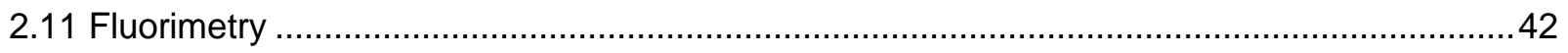

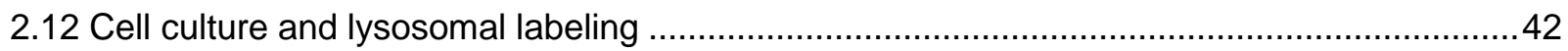

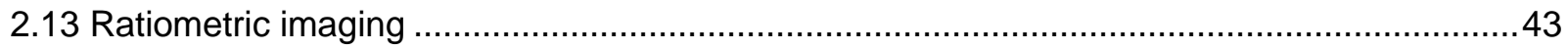

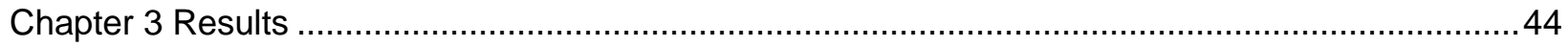

3.1 Characterization and the importance of homomeric Vac14 ........................................... 45

3.1.1 Co-immunoprecipitation to show Vac14 self-interaction in-vivo using Vac14 truncations . 45

3.1.2 in-vitro recombinant Vac14 interaction with Vac14 truncations and point mutants............49

3.1.3 Characterization of recombinant Vac14 multimer by fast protein liquid chromatography .. 51

3.1.4 Characterization of recombinant Vac14 multimer by differential velocity ultracentrifugation

3.1.5 Co-Immunoprecipitation to show Vac14 self-interaction is required for interaction with Fig4

3.1.6 Fluorescence microscopy to show Vac14 self-interaction is required for the rescue of

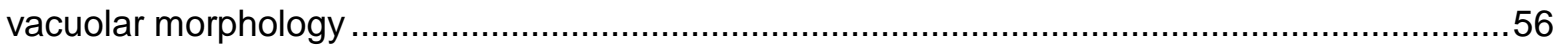

3.2 The role of Ptdlns $(3,5) \mathrm{P}_{2}$ in vacuolar and lysosomal acidification .....................................58

3.2.1 Loss of quinacrine accumulation in vacuoles of Ptdlns3,5 $\mathrm{P}_{2}$-deficient yeast...................58

3.2.2 Vacuolar accumulation of Mup1-pHluorin ...................................................................60

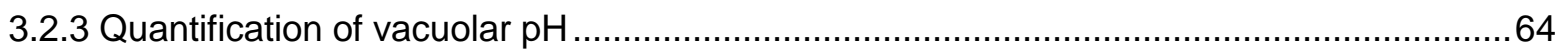

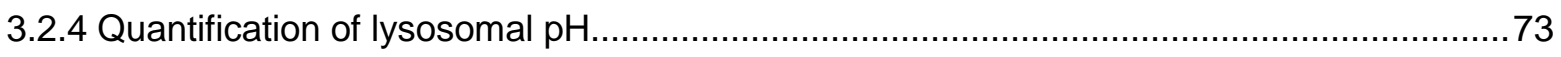

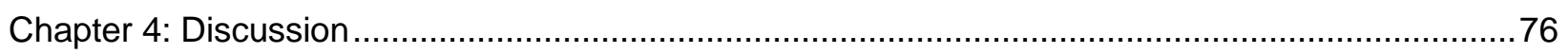

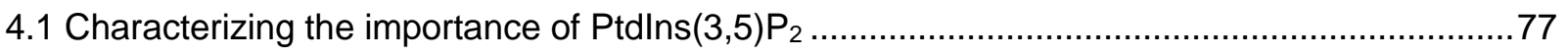

4.1.1 Vac14 homomerization via the C-terminus ................................................................. 78

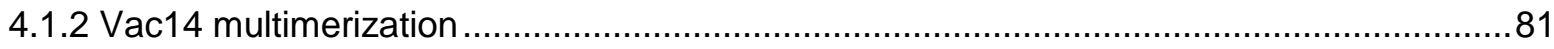

4.1.3 Vac14 multimer is required for the formation of the Fab1 complex and regulation of

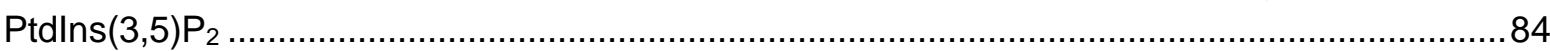

4.2 Ptdlns $(3,5) \mathrm{P}_{2}$ is not required for the regulation of steady-state vacuolar and lysosomal

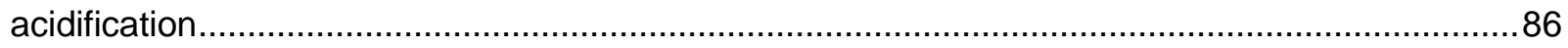

4.2.1 Ptdlns $(3,5) \mathrm{P}_{2}$ is not required for vacuolar acidification under basal conditions in yeast....86

4.2.2 The lysosomal $\mathrm{pH}$ remains acidic in the presence of PIKfyve inhibitors ...........................89

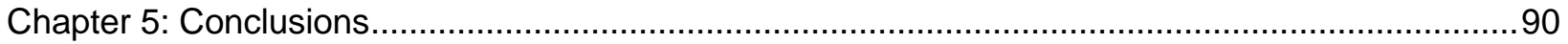

5.1 The Vac14 homodimer complex is required for the regulation of Ptdlns $(3,5) \mathrm{P}_{2} \ldots \ldots \ldots \ldots \ldots \ldots . . . . . . . . . .1$

$5.2 \mathrm{Ptd} \operatorname{lns}(3,5) \mathrm{P}_{2}$ is not required for the regulation of steady-state vacuolar and lysosomal

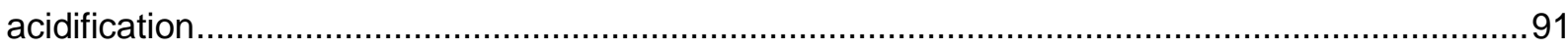

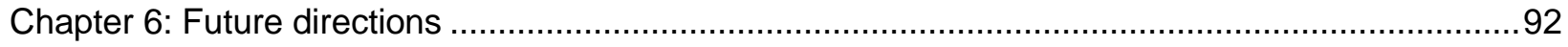

6.1 Identify the factor that affects vacuolar accumulation of quinacrine ....................................93 
6.2 Characterize the interaction of recombinant human Vac14 (ArPIKfyve) containing point mutations recently identified in patients with neurodegenerative diseases......

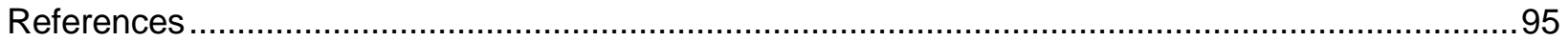




\section{List of Figures}

Figure 1. 1 An overview of the cellular compartments. .......................................................... 4

Figure 1. 2 A schematic of the main components involved in the regulation of $\mathrm{pH}$ homeostasis.. 6

Figure 1. 3 A diagram of the V-ATPase ........................................................................ 12

Figure 1. 4 Phosphatidylinositol and its derivatives........................................................ 15

Figure 1. 5 Anion-exchange HPLC chromatograms displaying levels of phosphoinositides after

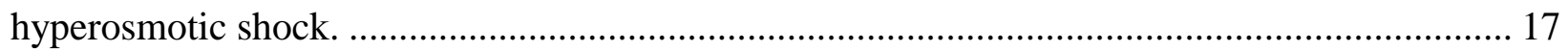

Figure 1. $6 \mathrm{PtdIns}(3,5) \mathrm{P}_{2}$ is required for vacuolar/endolysosomal morphology ....................... 18

Figure 1. $7 \mathrm{Fab} 1$ is required for the accumulation of quinacrine ........................................ 21

Figure 1. 8 The regulatory pathway of phosphatidylinositol 3,5-bisphosphate ........................ 22

Figure 1. 9 Localization of Vac14 and Fig4 to the vacuolar membrane requires Fab1.............. 29

Figure 1. 10 Vac14 interaction with Fig4 is required for association with Fab1........................ 30

Figure 1. 11 Fab1 interaction with Vac14 and Fig4 requires the GroL-related domain of Fab1. 31

Figure 1. 12 Vac14 homomeric interaction. .................................................................. 33 


\section{List of Abbreviations}

A280 - Absorbance at 280nm

AEBSF - 4-(2-aminoethyl) benzene sulfonyl fluoride hydrochloride

ALS - amyotrophic lateral sclerosis

$\mathrm{BME}-\beta$-mercaptoethanol

CCCP - carbonyl cyanide m-chlorophenyl hydrazine

cDCFDA - 5-(and 6-) carboxy-2',7'-dichlorofluorescein diacetate

CMAC - 7-amino-4-chloromethylcoumarin

CMT4J - Charcot-Marie-Tooth disorder type 4J

Con A - concanamycin A

CPS - carboxypeptidase S

CPY - carboxypeptidase Y

DIC - differential interference contrast

DMEM - Dulbecco's modified Eagle's medium

$\mathrm{EE}$ - early endosome

EEA1 - early endosome antigen 1

EGFP - enhanced green fluorescence protein

ENTH - Epsin $\mathrm{NH}_{2}$-terminal homology

ESCRT - endosomal sorting complexes required for protein transport

FITC - fluorescein isothiocyanate

FPLC - fast protein liquid chromatography

FYVE - Fab1, YOTB, Vac1, EEA1

GFP - green fluorescence protein 
HEAT - Huntingtin, Elongation factor 3, regulatory subunit A of Protein phosphatase 2A, and protein kinase TOR

HIS - histidine

ILVs - intraluminal vesicles

IP - immunoprecipitation

LAMP1 - lysosomal-associated membrane protein 1

LB - Luria Broth

LE - late endosome

MVB - multivesicular body

PAT - proton-assisted amino acid transporters

$\mathrm{PCR}$ - polymerase chain reaction

$\operatorname{Pr} \mathrm{A}$ - proteinase $\mathrm{A}$

PtdIns - phosphatidylinositol

PtdIns(3)P - phosphatidylinositol 3-phosphate

PtdIns(3,5) $\mathrm{P}_{2}$ - phosphatidylinositol 3,5-bisphosphate

PtdInsPs - phosphoinositides

PolyP - polyphosphates

PVDF - polyvinylidene fluoride

PX - phox homology

$\mathrm{SC}$ - synthetic complete

SD - synthetic-deficient

SDS-PAGE - sodium dodecyl sulfate-polyacrylamide gel electrophoresis siRNA - small interference RNA 
SNAREs - soluble NSF attachment protein receptor

TBS - tris buffered saline

TCA - trichloroacetic acid

TRPML - transient receptor potential mucolipin family of ion channels

V-ATPase - vacuolar-type proton ATPase

YPD - yeast extract, peptone, and D-gluco 
Chapter 1 Introduction 
The lysosome is the primary degradation and recycling center of cells. Lysosomes are membrane-bound organelles that contain various hydrolytic enzymes. These enzymes include proteases, phosphatases, and lipases that function to degrade macromolecules (5). The turnover of macromolecules into amino acids, fatty acids, and carbohydrates are recycled for cellular synthesis (6). Lysosomal proteins are initially synthesized in the rough endoplasmic reticulum and undergoes various post-modifications while they are transported through the Golgi (6). The modified lysosomal proteins are eventually translocated to the lysosomes. Macromolecules destined for lysosomal degradation are transported to the lysosome either via the endocytic pathway or autophagy, while the degraded products can be exported from the lysosome into the cytosol via various lysosomal membrane transporters for cellular synthesis (7-10).

Defects that affect proper functioning of lysosomal enzymes, transport of cargos into and out of the lysosome can result in lysosomal storage diseases. Lysosomal storage diseases are a group of genetic disorder that result in the accumulation of undegraded macromolecules in the lysosome. The excessive build-up of cellular components in the lysosome can have severe pathological consequences $(6,11)$. For example, Niemann-Pick diseases are a group of genetic lysosomal storage disorder that result from deficiency in the activity of sphingomyelinase or defective transport of cholesterol leading to build-up of lysosomal sphingomyelin and cholesterol. Different types of Niemann-Pick diseases can manifest into mild to severe neurodegeneration (12, $13)$.

The lysosome is the converging point of multiple pathways including endocytosis and biosynthesis, therefore, defects in the function of different organelles and membrane trafficking can affect the proper function of lysosomes. 


\subsection{Organelle compartmental acidification}

In eukaryotes, cellular processes are carried out in specialized membrane-bound compartments to maintain efficiency and precision. The different compartments not only generate a confined space for specialized reactions, they are also $\mathrm{pH}$-regulated to provide an environment best suited for reactions and enzymes that are highly sensitive to $\mathrm{pH}$. For example, in the endocytic pathway, the starting compartment is generated by uptake of a variety of ligands, particles, and fluids in the extracellular space into small membrane-bound vesicles termed early endosomes (EE). These EEs rapidly acidify to a $\mathrm{pH}$ range of $6.5-6.0$ as they mature and transport cargos to the late endosomes (LE) (Figure 1.1) $(14,15)$. This acidification is a key process for sorting of cargos for recycling to the plasma membrane or continuing through the endocytic pathway. The change in $\mathrm{pH}$ in EEs causes dissociation of specific ligands from their receptors, which determines their fate. For example, transferrin receptor and its ligand, transferrin, are recycled back to the plasma membrane, while the iron remains in the endocytic vesicle $(16,17)$. Membrane receptors destined for degradation are ubiquitinated and are further sorted into intraluminal vesicles (ILV) by a family of conserved proteins known as the ESCRT (endosomal sorting complexes required for protein transport) machinery. The formation of ILVs is an indication of maturation of EEs into LEs, also known as the multivesicular body (MVB) (Figure 1.1) $(18,19)$. The $\mathrm{pH}$ of the endocytic vesicle progressively decreases in the endocytic pathway and reaches a $\mathrm{pH}$ of $<5$ as it matures into a lysosome (Figure 1.1). The highly acidic lysosome is required for the activation of lysosomal hydrolases for the degradation of cargos, as well as the clearance of pathogens. In contrast to the acidic organelles, some organelles maintain a less acidic $\mathrm{pH}$, such as the endoplasmic reticulum $(\sim \mathrm{pH}$ 7.2) and the Golgi ( pH 6.5) (Figure 1.1) (20-22). The less acidic environment in these organelles helps facilitate the biosynthesis, post-modifications, and the secretory transport of cellular components. 


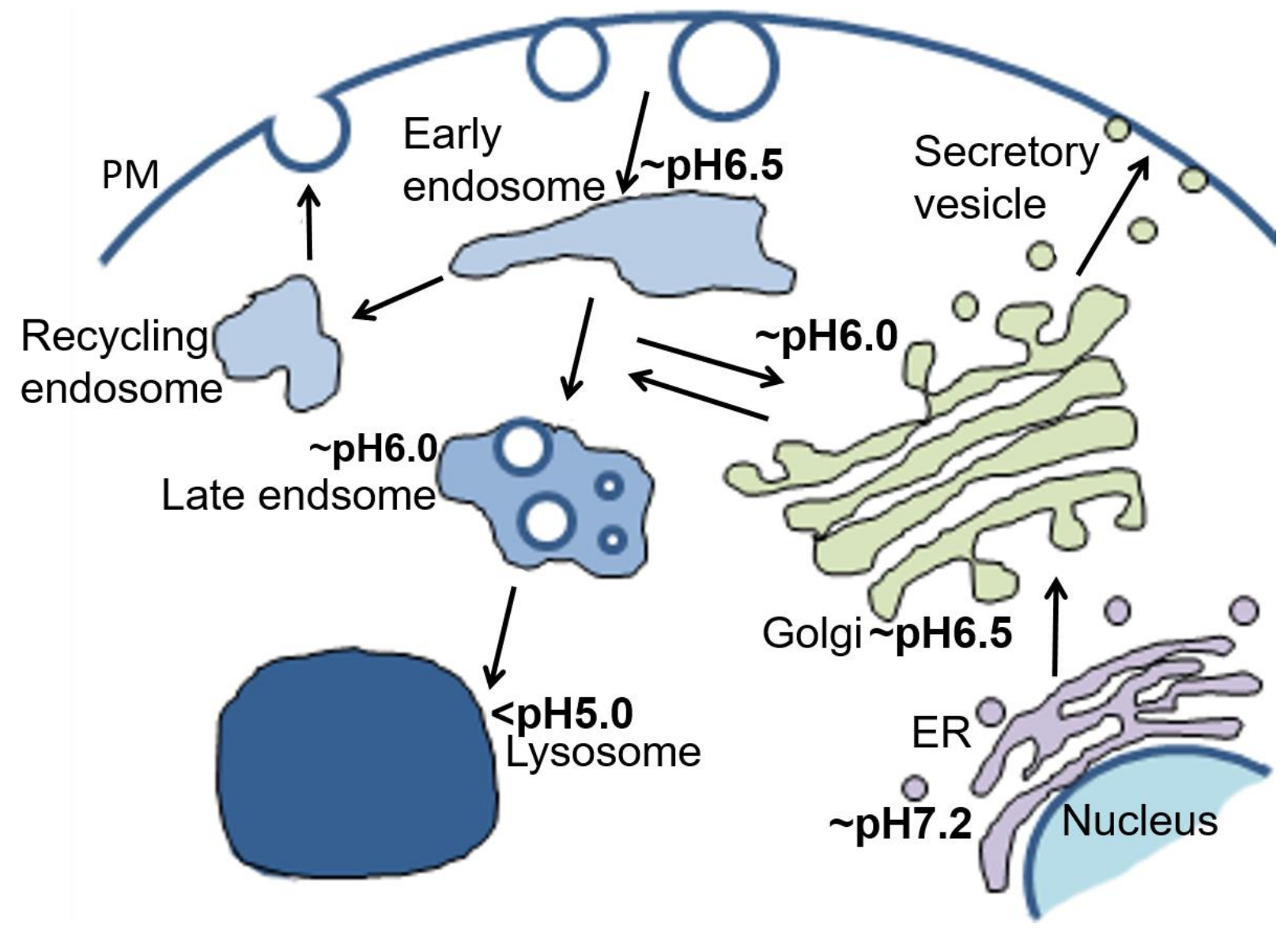

Figure 1. 1 An overview of the cellular compartments.

The $\mathrm{pH}$ of organelles rapidly decreases as they progress through the endocytic or the secretory pathway. The regulated acidity is required for specific cellular reactions in different compartments. 


\subsubsection{The regulation of $\mathrm{pH}$ homeostasis}

The $\mathrm{pH}$ homeostasis of organelles is regulated and maintained by a number of processes, such as proton pumping, exchange of counter-ions, and proton leaks. Membranes are intrinsically permeable to protons permitting movement of protons into and out of organelles (Figure 1.2) (23, 24). However, proton permeability is governed by the transmembrane proton gradient and membrane lipid composition. The proton gradient is generated by the balance of a combination of active proton pumping and counter-ion movement across the membrane. The vacuolar-type proton-ATPase (V-ATPase) plays a crucial role in the acidification of organelles by actively pumping cytosolic protons into the organelle lumen. This action generates a large luminal positive membrane potential. Through pharmacological approaches, ion channels were found to play a major role in the acidification of endosomes and lysosomes (25). For example, the chloride ion channel allows the influx of chloride to help dissipate the positive membrane potential. Although the extent of counter-ion movement on $\mathrm{pH}$ regulation varies between organelles, they often play a role in facilitating the continuous action of vacuolar-type proton-ATPase (V-ATPase). In addition, the $\mathrm{Na}^{+} / \mathrm{K}^{+}$-ATPase, transports three sodium into and two potassium out of the organelle. The build-up of sodium drives the $\mathrm{Na}^{+} / \mathrm{H}^{+}$-antiporter to further promote proton influx and the acidification of the organelle (Figure 1.2) (24, 26). Disruption of $\mathrm{pH}$ homeostasis through pathogenesis or protein dysfunction can compromise proper organelle and enzyme functions, and manifest into several diseases. 


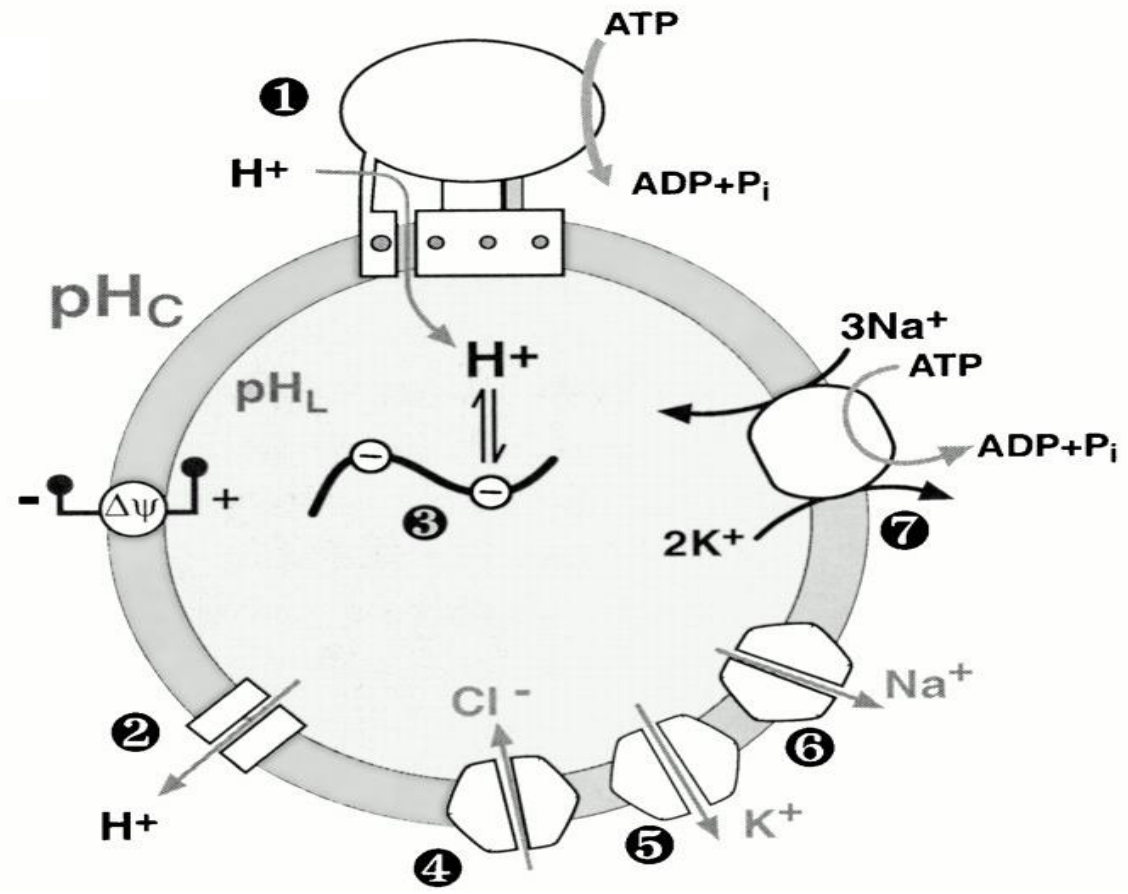

Figure 1. 2 A schematic of the main components involved in the regulation of $\mathrm{pH}$ homeostasis.

(1) The V-ATPase that acidifies the lumen by transport of proton at the expense of ATP; (2) represents the intrinsic proton leakage property of the membrane; (3) polyphosphates that readily protonate or deprotonate, can either release or help sequester protons and affect luminal $\mathrm{pH}$; (4 -6) example of ion channels that can affect the membrane potential; (7) the Na/K-ATPase can act to limit or counterbalance the acidification by the V-ATPase to control for luminal acidity (Image taken with permission from Grabe, M., and Oster, G., 2001). 


\subsubsection{The importance of lysosomal/vacuolar acidification: Degradation}

The importance of compartmental acidification is most prominent in the lysosome/vacuole,

where its acidic environment is widely known for its role in protein and organelle degradation.

The yeast vacuole is functionally similar to the mammalian lysosome and is often used as a model for studying lysosomal function. Studies with the vacuole have provided a vast array of insights into the mechanisms underlying the endocytic pathway (5).

As mentioned previously, many lysosomal/vacuolar acid hydrolases require the highly acidic environment of the lysosome/vacuole in order to function optimally. Many acid hydrolases exist as proenzymes, an inactive form of the enzyme, and are only activated upon arrival in the lysosomal/vacuolar lumen. To prevent the premature activation of proenzymes and degradation of cellular components outside of the lysosome/vacuole, the activation of proteases involves a complex and highly regulated cascade. For example, both proteinase A (PrA) and the carboxypeptidase $\mathrm{Y}(\mathrm{CPY})$ first transport to the vacuole as inactive proenzymes. The low vacuolar $\mathrm{pH}$ is required for optimal autocatalytic cleavage of proPrA into $\operatorname{Pr} \mathrm{A}$. $\operatorname{Pr} \mathrm{A}$ is an endopeptidase that hydrolyzes and degrades general proteins, which includes hydrolyzing other proenzymes, including proCPY to convert it into active CPY for peptide degradation (27-29). Similarly, cathepsin D is a soluble lysosomal aspartic endopeptidase in mammalian cells that is an ortholog to PrA in yeast. The acidity of the lysosome activates the autocatalytic activity of Cathepsin D into its active form for general protein degradation (30-32). In addition to the activation of proenzymes, the low lysosomal/vacuolar $\mathrm{pH}$ also helps to stabilize active enzymes to allow for optimal function. Defects in lysosomal acidification can lead to failure of proteases to clear damaged proteins and organelles. Ultimately, this will lead to the appearance and build-up of aggregated damaged cellular components. Accumulation of aggregated proteins is often related to the onset of diseases, such as a number of lysosomal storage diseases. 


\subsubsection{The importance of lysosomal/vacuolar acidification: Vesicular transport and}

fusion

Lysosomal/vacuolar acidification does not only affect the optimal degradation of particles and cellular components; it is also important for vesicle transport to the lysosome/vacuole. Lysosome/vacuole continuously receive cargos such as MVBs carrying proteins destined for degradation. In addition to MVBs, vesicles from Golgi carrying lysosomal/vacuolar hydrolases may also fuse with either the MVBs or directly with lysosome/vacuole (33). Furthermore, autophagosomes generated de novo in the cytosol carrying cytosolic damaged proteins or organelles are also transported to the lysosome/vacuole for degradation (34). However, in order to deliver the content of these vesicles into the lysosome/vacuole, these incoming vesicles (mentioned above) are required to undergo membrane fusion with the lysosomal/vacuolar membrane. Membrane fusion is a highly regulated process that involves membrane recognition, attachment and eventual fusion of the incoming vesicle membrane with the membrane of the target organelle (35). The fusion of membranes requires the combined action of Rab GTPases and a protein complex composed of protein effectors and tethering factors (35). Among the proteins involved in membrane fusion, SNARE (soluble NSF attachment protein receptor) are membrane proteins that are anchored to both the membrane of the incoming vesicle (v-SNARE) and the membrane of the target organelle (t-SNARE). The interaction between v-SNAREs and t-SNAREs is required for docking of the vesicle to the organelle, and fusion of the two membrane compartments (36-39). Upon v- and t-SNARE interaction, the lysosome/vacuole releases $\mathrm{Ca}^{2+}$ to destabilize the membrane bilayers and facilitate further interaction and eventual fusion of the two membranes (40-42). TRPML, a member of the transient receptor potential mucolipin family of ion channels, may be responsible for the release of $\mathrm{Ca}^{2+}$ from lysosome/vacuole during membrane fusion. The use of TRPML knockout and non-functional TRPML mutants in a number of studies revealed a 
delay in cargo transport to the lysosome and also an abnormal accumulation of LE structures (4348). Together, these suggest that TRPML may be responsible for the efflux of $\mathrm{Ca}^{2+}$ from lysosome/vacuole that drives membrane fusion and cargo deposition into lysosome/vacuole (4348). Lysosomal/vacuolar acidification plays a role in membrane fusion by facilitating the storage of $\mathrm{Ca}^{2+}$ for the release of $\mathrm{Ca}^{2+}$ during SNARE assisted membrane fusion. Storage of $\mathrm{Ca}^{2+}$ is accomplished by transporters, such as the $\mathrm{Ca}^{2+} / \mathrm{H}^{+}$exchanger, Vcx1. Vcx1 requires the high concentration of lumenal $\mathrm{H}^{+}$generated by the V-ATPase to drive exchange of $\mathrm{Ca}^{2+}$ into the lumen (49). This an example where the V-ATPase and counter-ion exchangers are inter-dependent for their function.

\subsubsection{The importance of lysosomal/vacuolar acidification: Storage of metabolites}

The lysosome/vacuole is also a storage compartment for metabolites, such as amino acids

and metals. Storage of metabolites is often accomplished by a number of transporters. These transporters include both importers and exporters, and many require the proton gradient (pHvacuole $=\sim 4.8$ and pHcytosol $=\sim 7.2)$ to drive their transport. For example, metals such as zinc, are important catalytic cofactors but an excess of cytosolic level can be toxic to the cell (50, 9). Therefore, the vacuole serves as a site for sequestering zinc and other metals to control their cytosolic levels. The vacuolar membrane contains a number of transporters for the uptake of metals and they are often metal/ $\mathrm{H}^{+}$antiporters, where the transport of proton down its concentration gradient drives the transport of metal into the vacuolar/lysosomal lumen (50-52).For example, $\mathrm{Zrc1}$ is a vacuolar zinc/proton antiporter that is responsible for vacuolar sequestration of zinc (50, $51)$.

Degraded products such as free amino acids from lysosomal/vacuolar degradation are often stored in the lysosome/vacuole. These degraded products can be exported to the Golgi via retrograde trafficking or released into the cytosol for biosynthesis of new cellular components. 
Especially during cell growth and survival, where free cytosolic amino acids are depleted, protonassisted amino acid transporters (PAT) on lysosomal/vacuolar membrane are responsible for the release of free amino acids into the cytosol. PATs are cotransporters, where the transport of $\mathrm{H}^{+}$ down its concentration gradient into the cytosol drives the cytosolic transport of free amino acids $(53,54)$. For example, PAT1 is a symporter that transports an amino acid, such as Ala, Pro, or Ser, along with a proton, in a 1:1 stoichiometry (55). Therefore, the continuous function of PAT also relies on the establishment of the proton gradient by the V-ATPase. In contrast to the cytosolic transport of amino acids, metabolites and amino acids can also be transported into the vacuolar/lysosomal lumen in a similar manner using the proton gradient by proton-amino acid anti-transporters. For example, the Vba family of amino acid transporters is responsible for the anti-transport of His, Lys, Arg into the vacuole and $\mathrm{H}^{+}$out into the cytosol $(8,10)$.

\subsubsection{The regulation and function of V-ATPase}

The V-ATPase is the main electrogenic pump on the vacuolar membrane that transports protons from the cytosol into the lumen using the energy released from ATP hydrolysis. The action of ATP hydrolysis and proton transport are performed by two distinct multi-subunit complexes of the V-ATPase: a catalytic complex $\left(\mathrm{V}_{1}\right)$ and a transmembrane complex $\left(\mathrm{V}_{0}\right)$, respectively (Figure 1.3) $(56,57)$. The precise mechanism of action of the V-ATPase remains elusive, but nevertheless, a speculative model has been proposed based on data from numerous studies. Importantly, the $\mathrm{V}_{1}$ complex contains a core mainly composed of three copies of the Vma1-Vma2 dimer. The Vma1 and Vma2 both contain a 'P-loop' sequence (GXXXXGKT) known to bind ATP for hydrolysis $(58,59)$. Other subunits are pivotal to the assembly and stabilization of the $\mathrm{V}_{1}$ and $\mathrm{V}_{0}$ association, such as $\mathrm{Vma}$, an integral component of $\mathrm{V}_{1}$, and $\mathrm{Vma5}$ and Vma13, which are peripheral to the core $\mathrm{V}_{1}$ complex and together form a stable interaction with the $\mathrm{V}_{0}$ complex (Figure 1.3) (60). 
Vph1 is a subunit of the $\mathrm{V}_{0}$ complex specific to the V-ATPase found on the vacuolar membrane. Vph1 contains an N-terminal hydrophilic domain residing on the cytosolic side for association with the $\mathrm{V}_{1}$ complex and a $\mathrm{C}$-terminal hydrophobic transmembrane domain allowing it to cross the vacuolar membrane to interact with the $V_{0}$ subunits and together form the $V_{0}$ complex (61). ATP hydrolysis by the $\mathrm{V}_{0}$ core leads to a conformational change that consequently drives cytosolic protons down through the $\mathrm{V}_{0}$ core into the vacuolar lumen (Figure 1.3) $(56,60,62)$. The Vph1 subunit acts to couple the ATP hydrolysis of the $V_{1}$ complex to the transfer of protons from the cytosolic side into the vacuolar lumen. A related protein Stv1 has the same function as the Vph1 but is specific to V-ATPase found on the Golgi and plasma membrane (63). Disruption of Vph1 results in partial loss of the $\mathrm{V}_{0}$ complex and vacuolar acidification. However, overexpression of Stv1 was able to complement the loss of Vph1. This suggests that although the Vph1 and Stv1 are localized to distinct cellular compartments, they can compensate for one another under stress conditions (63).

$\mathrm{V}_{0}$ and $\mathrm{V}_{1}$ alone are not capable of ATP hydrolysis or proton transport, respectively. Therefore, regulating the assembly and disassembly of the V-ATPase is an effective means of controlling the activity of the V-ATPase (64). Although the precise signaling pathway for the assembly and disassembly of the V-ATPase remains unknown, the physiological condition of the cell appears to have an effect. For example, when yeast is switched to their less favorable carbon source, such as raffinose or galactose, there is a rapid dissociation of the $V_{0}$ and $V_{1}$ complexes, but the two sub-complexes will quickly reassemble when the carbon source is switched back to glucose (62). Extracellular pH and salt concentration can also affect the activity and stability of the V-ATPase. For example, under hypertonic conditions, there is an increase in V-ATPase subunit production which leads to an increase in V-ATPase activity (65). The V-ATPase often works in 
conjunction with counter-ion exchangers. Therefore, the increase in V-ATPase activity due to elevated salt level may enhance the sequestration activity of counter-ion exchangers to re-establish the osmotic homeostasis.

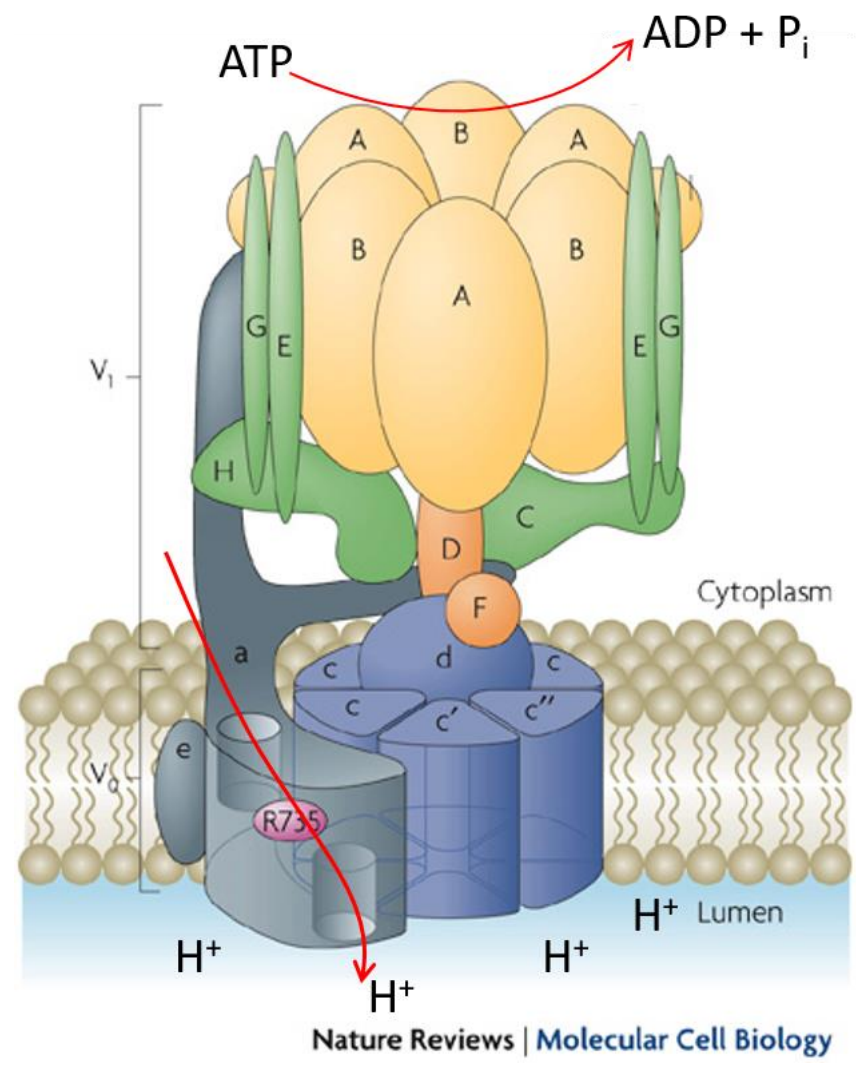

\begin{tabular}{|l|l|l|l|l|}
\hline \multicolumn{2}{|l|}{ V1 domain } & & \multicolumn{2}{l|}{ V0 domain } \\
\hline Subunit & Yeast gene & & Subunit & Yeast gene \\
\hline A & VMA1 & & a & VPH1/STV1 \\
\hline B & VMA2 & d & VMA6 \\
\hline C & VMA5 & e & VMA9 \\
\hline D & VMA8 & c & VMA3 \\
\hline E & VMA4 & VMA7 & VMA11 \\
\hline F & CM & VMA16 \\
\hline G & VMA10 & & \\
\hline H & VMA13 & & \\
\hline
\end{tabular}

Figure 1. 3 A diagram of the V-ATPase.

The core of the $\mathrm{V}_{1}$ cytosolic complex is composed of a hexameric Vma1-Vma2 (A-B) dimer. The $\mathrm{V}_{1}$ core is responsible for the binding and hydrolysis of ATP into ADP and $\mathrm{P}_{\mathrm{i}}$. The $\mathrm{V}_{1}$ complex also consists of a number of subunits $(\mathrm{E}-\mathrm{H})$ necessary for the assembly and functioning of $\mathrm{V}_{1}$ with $\mathrm{V}_{0}$. ATP hydrolysis by the $\mathrm{V}_{1}$ complex drives the transfer of $\mathrm{H}^{+}$from the cytoplasm into the vacuolar lumen. The $\mathrm{H}^{+}$in the cytoplasm passes through the $\mathrm{V}_{0}$ complex, mainly the $\mathrm{Vph} 1$ and the ring of c, c' and c" subunits, before being deposited in the vacuolar lumen. (Image modified with permission from Forgac, M., 2007). 
The V-ATPase is the central player in organelle acidification which impacts a variety of cellular pathways. However, in addition to organelle acidification, proper organelle functions also require a collection of organelle-specific protein effectors and phosphoinositides. The combination of protein effectors and phosphoinositides facilitate cellular functions such as signalling, and protein and membrane trafficking.

\subsection{Phosphoinositides}

Phosphatidylinositol (PtdIns) is a phospholipid that contains a D-myo-inositol head group and two fatty acids connected to a glycerol backbone at the positions, C3, C1 and C2 (respectively) (Figure 1.4A). The fatty acid compositions of PtdInsPs are mainly in the form 1-stearoyl, 3arachidonoyl polyunsaturated fatty acid (66). The difference in saturation level of the fatty acid chains may affect their interaction with specific kinases/phosphatases and its downstream signaling events (66-68). The inositol head group of PtdIns can be differentially phosphorylated at its 3-, 4-, and/or 5-OH positions to generate seven different species of phosphoinositides (PtdInsPs) (Figure 1.4B). The seven PtdInsPs collective exist in low abundance, and only comprise approximately $15 \%$ of total phospholipids (69). Each PtdInsP is localized to distinct organelles to perform specialized functions. The cell undergoes continuous trafficking of cargos from one organelle to another. Therefore, in order to maintain organelle identity in the midst of all the trafficking events, PtdInsPs undergo rapid interconversions by specific kinases and phosphatases. For example, as the endosome matures into lysosomes, phosphatidylinositol 3-phosphate [PtdIns(3)P] on the endosome is rapidly converted into phosphatidylinositol 3,5-bisphosphate [PtdIns(3,5) $\mathrm{P}_{2}$ ] found on the lysosome by the PtdIns(3)P 5-kinase, PIKfyve (mammalian orthologue of the yeast Fab1) (70). 
Phosphoinositides regulate organelle functions mainly by direct interaction and recruiting distinct sets of protein effectors. For example, PtdIns(3)P is mainly found on the early endosomes, with a small subset found on phagosomes, autophagosomes, and retrograde vesicles (71). Although PtdIns(3)P is present on different organelles, PtdIns(3)P recruits a different set of effectors depending on the organelle where it is located. The ability of the same PtdInsP to recruit different sets of effectors is due to coincidence detection. PtdInsP in conjunction with another protein, most often a GTPase that is native to the organelle, recruit protein effectors that are specific for the organelle. The use of a combination of PtdInsPs and GTPases offers increased specificity and binding affinity of protein effectors. For example, the tethering protein, early endosome antigen-1 (EEA1) is specifically recruited to the early endosomes to mediate endocytic fusion by $\operatorname{PtdIns(3)P~}$ and GTPase Rab5. EEA1 contains a C-terminal FYVE (named after first four proteins to contain this region: Fab1, YOTB, Vac1, EEA1) domain that specifically interacts with PtdIns(3)P. However, this FYVE domain alone is not sufficient for efficient EEA1 endosomal recruitment and fusion events (72-74). In addition to the FYVE domain, EEA1 also contains a 30-amino acids region upstream of the FYVE domain that interacts with an activated Rab5 and this interaction is critical for endocytic vesicle fusion (75). Together, PtdIns(3)P and Rab5 in concert interact with EEA1 and enhance EEA1 recruitments to early endosomes $(74,75)$.

In comparison, PtdIns(3)P on retrograde vesicles recruits protein effectors such as Vps5 and Vps17 (mammalian homolog: Snx1 and Snx2, respectively) to form a retromer complex for trafficking to the late-Golgi (76). Interestingly, Vps5 and Vps17 contain a Phox homology (PX) domain for interaction with PtdIns(3)P. Different PX domains alone were shown to have different affinities for PtdIns(3)P (77). Proteins with a PX domain often contain other protein binding domains to increase their affinity for localizing to specific organelles. Vps5 is found to form a 
complex with Vps17, and they both contain a PX domain. The combination of two PX domains offers an increased binding affinity for PtdIns(3)P to facilitate retrograde vesicle formation and trafficking on endosomal membranes (76-78). Thus, the differential binding of FYVE and PX to PtdIns(3)P can be attributed to their spatiotemporal location and their requirement for additional different binding partners (79).

A

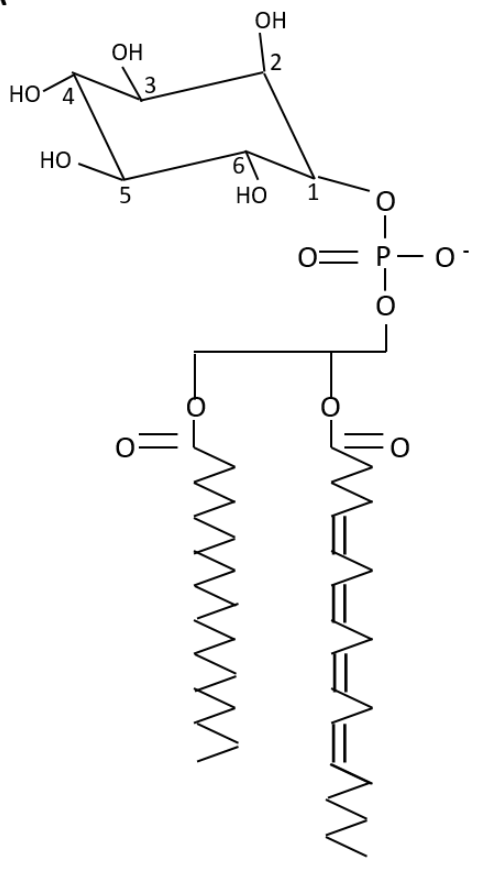

Phosphatidylinositol

(Ptdlns)
B

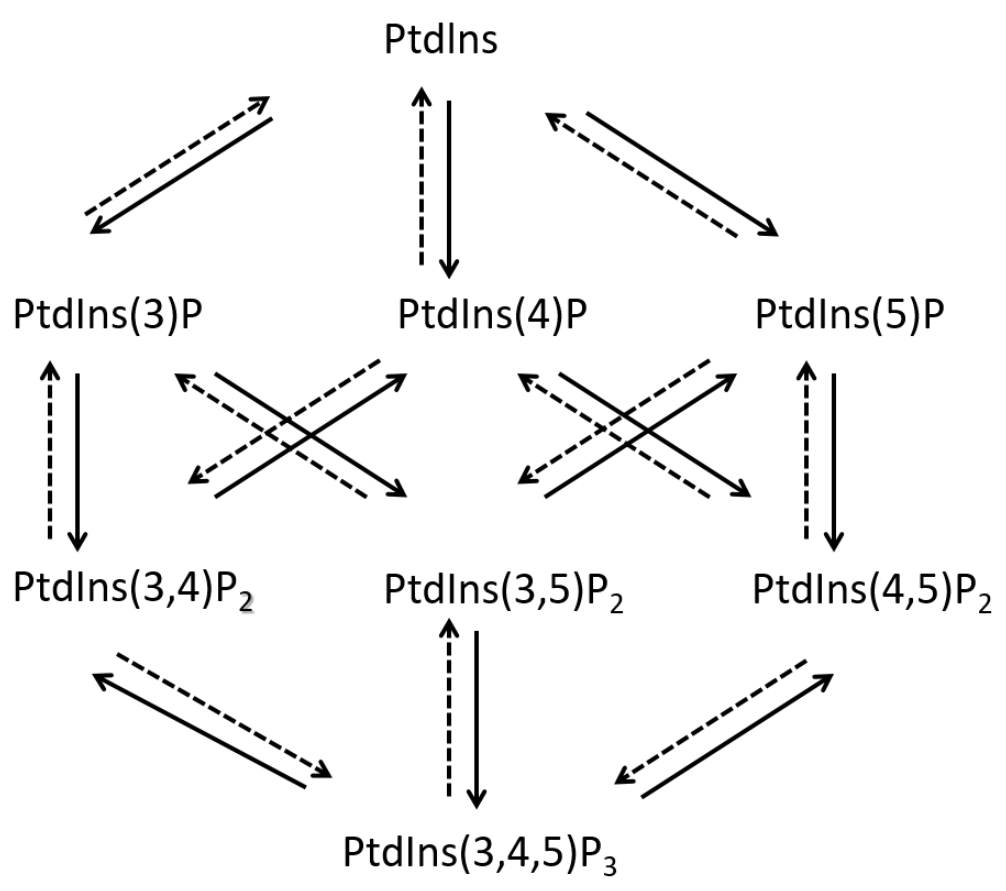

Figure 1. 4 Phosphatidylinositol and its derivatives.

(A) Phosphatidylinositol (PtdIns) is composed of an inositol head group connected to two fatty acids through a glycerol backbone. (B) Reversible phosphorylation by kinases and phosphatase at the 3-, 4-, and/or 5-OH position generates seven different species of phosphoinositides (69). 


\subsubsection{Phosphatidylinositol-3,5-bisphosphate}

$\operatorname{PtdIns}(3,5) \mathrm{P}_{2}$ is the least abundant form of phosphoinositides, comprising approximately

$0.1 \%$ of total phosphoinositides in yeast and mammalian cells $(80-82)$. PtdIns $(3,5) \mathrm{P}_{2}$ is found mainly on the lysosomal/vacuolar membrane and has been implicated in a variety of functions.

Due to the low abundance of PtdIns(3,5) $\mathrm{P}_{2}$ at basal level, PtdIns(3,5) $\mathrm{P}_{2}$ in Saccharomyces cerevisiae was initially discovered after exposure to hyperosmotic shock. During hyperosmotic shock, the level of $\operatorname{PtdIns}(3,5) \mathrm{P}_{2}$ increased dramatically by approximately 20 -fold, allowing detection of PtdIns(3,5) $\mathrm{P}_{2}$ (Figure 1.5) (81-83). Increased PtdIns(3,5) $\mathrm{P}_{2}$ during hyperosmotic stress was accompanied by fission of the vacuole into multiple smaller vacuolar compartments, while cells deficient in PtdIns(3,5) $\mathrm{P}_{2}$ display a single enlarged vacuole (Figure 1.6A) $(2,82,84)$. Under hyperosmotic shock, the vacuole losses water and in turn vacuolar volume in an attempt to regulate cellular osmolarity. Therefore, the increase in $\operatorname{Ptd} \operatorname{Ins}(3,5) \mathrm{P}_{2}$ level may help to regulate the vacuolar morphology when there is a change in vacuolar volume. Similar to the enlargement of the vacuole in PtdIns(3,5) $\mathrm{P}_{2}$-deficient yeast, mammalian cells also exhibit enlarged late endolysosomal structures when PtdIns(3,5) $\mathrm{P}_{2}$ synthesis is blocked by either drug inhibition or mutation in the catalytic domain of PIKfyve (Figure 1.6B) (1). Although Ptdins(3,5) $\mathrm{P}_{2}$ involvement in the regulation of hyperosmolarity in mammalian cells is not as apparent, hyperosmotic stress in differentiated mouse 3T3-L1 adipocytes induced a 10-fold increase in the level of PtdIns(3,5) $\mathrm{P}_{2}(85)$. However, the precise mechanism of how $\operatorname{PtdIns}(3,5) \mathrm{P}_{2}$ manipulates vacuolar size and morphology is still unknown. 

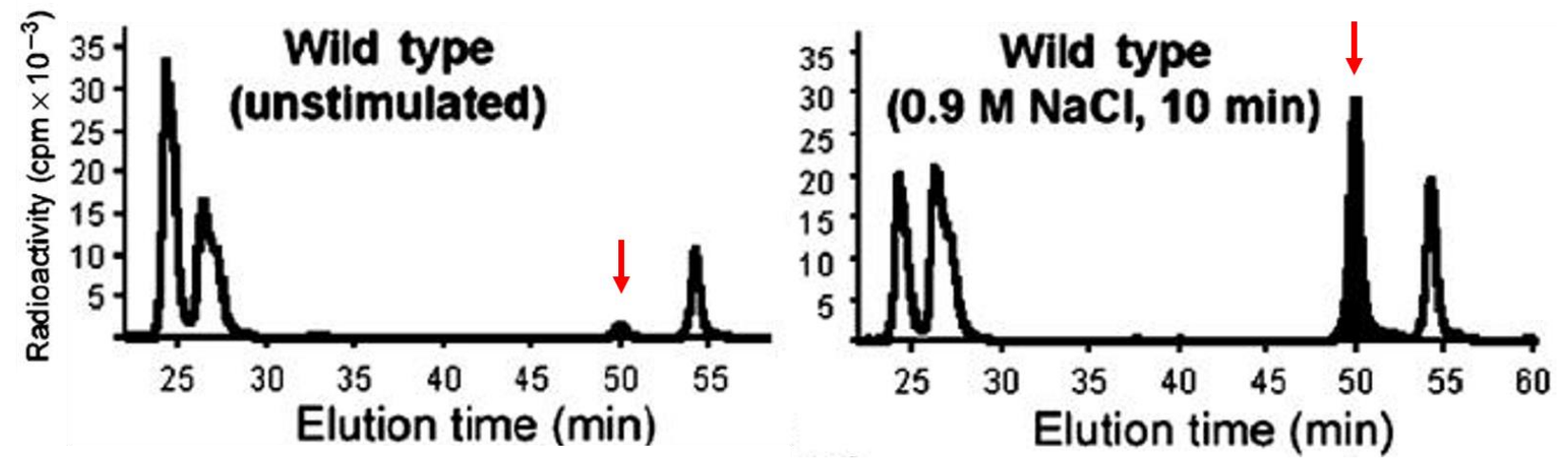

Figure 1. 5 Anion-exchange HPLC chromatograms displaying levels of phosphoinositides after hyperosmotic shock.

Cells were incubated with $\left[\mathrm{H}^{3}\right]$-inositol for labeling $\left[\mathrm{H}^{3}\right]$-PtdInsPs. Deacylated $\left[\mathrm{H}^{3}\right]$-PtdInsPs were fractionated and recorded as radioactive signal peaks in the order PtdIns(3)P, PtdIns(4)P, PtdIns(3,5) $\mathrm{P}_{2}$ (red arrow), and PtdIns(4,5) $\mathrm{P}_{2}$. Comparison between wild-type unstimulated (left) and wild-type (hyperosmotic shock) demonstrates a relative change in PtdInsPs (Image modified with permission from Dove, S. K., Piper, R. C., McEwen, R. K., et al, 2004). 
A
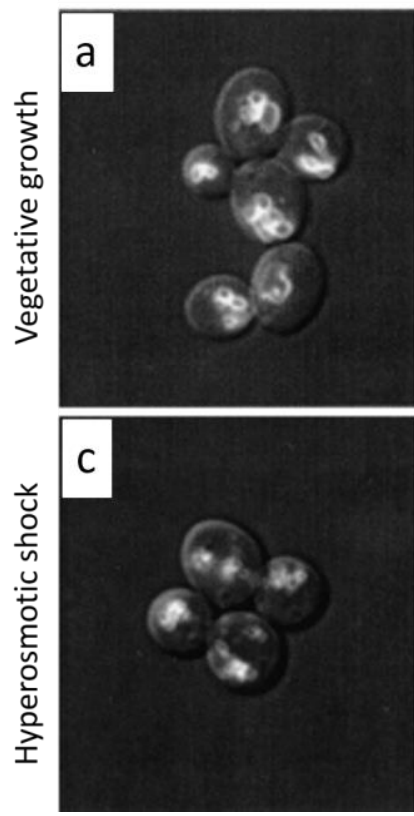

B
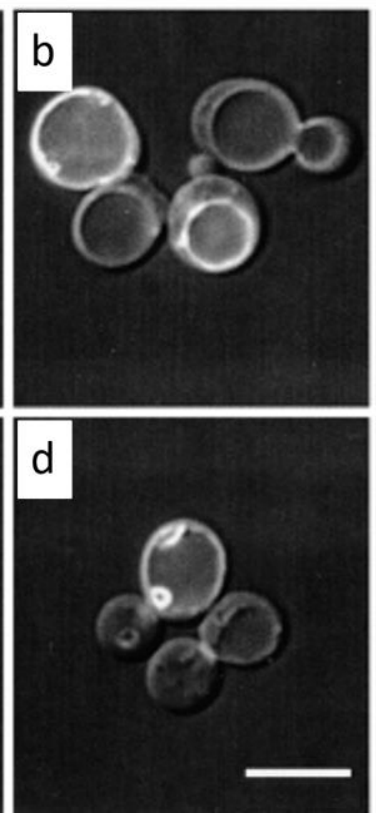
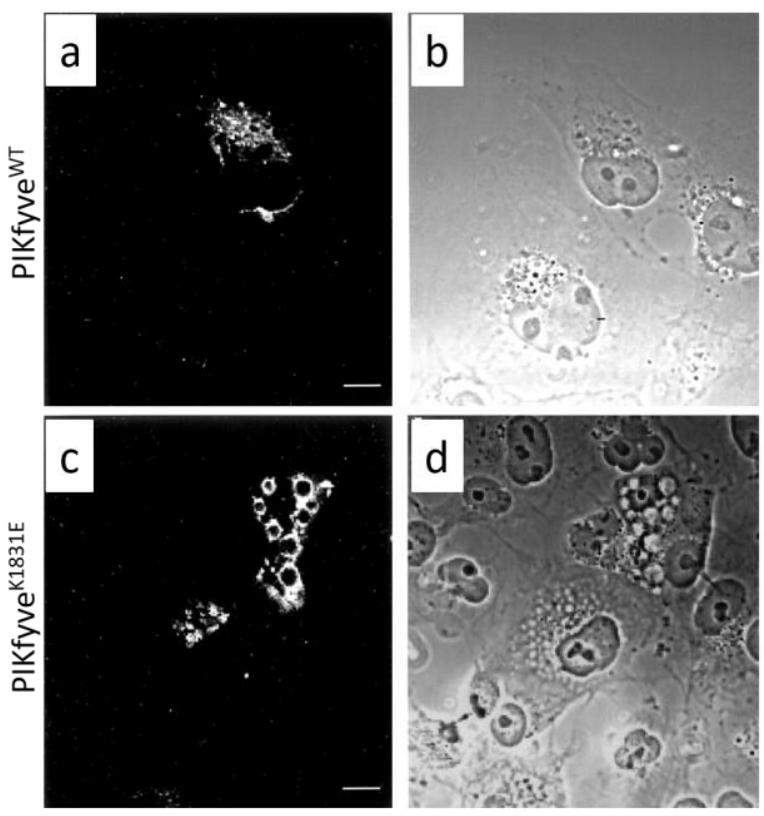

Figure 1. 6 PtdIns(3,5) $\mathrm{P}_{2}$ is required for vacuolar/endolysosomal morphology.

(A) Yeast cells labeled with FM4-64, a marker for visualizing vacuolar membrane. Wild-type yeast grown under normal vegetative condition contains $2-3$ vacuoles (a), while wild-type vacuoles undergo fission when grown under $0.4 \mathrm{M} \mathrm{NaCl}$ hyperosmotic shock (c). fablA, yeast deficient in PtdIns $(3,5) \mathrm{P}_{2}$, grown under both normal vegetative condition and $0.4 \mathrm{M} \mathrm{NaCl}$ hyperosmotic shock contain an enlarged vacuole ( $\mathrm{b}$ and $\mathrm{d}$, respectively) (Image modified with permission from Bonangelino, C. J., Nau, J. J., Duex, J. E., et al, 2002). (B) COS-7 cell expressing EGFP epitopetagged-PIKfyve $^{\mathrm{WT}}$, the mammalian PtdIns(3)P 5-kinase, are localized to small punctate endolysosomal structures (a), while EGFP epitope tagged-PIKfyve ${ }^{\mathrm{K} 1831 \mathrm{E}}$, which expresses a catalytic inactive PIKfyve, localized to enlarged endolysosomal structures (c), and their corresponding DIC images (b, d, respectively) (Image modified with permission from Ikonomov, O. C., Sbrissa, D., and Shisheva, A, 2001). 
In addition, $\operatorname{Ptd} \operatorname{Ins}(3,5) \mathrm{P}_{2}$ is also important in endocytic and retrograde trafficking to and from the vacuole/lysosome (86-88). For example, carboxypeptidase S (CPS) is a membrane associated vacuolar hydrolase that is transported from the Golgi to the vacuole via the CPY pathway, which passes through the late endosomal compartment. CPS on the late endosomal membrane is sequestered into ILV in MVB. Upon MVB fusion with vacuoles, CPS in ILV is exposed to the vacuolar lumen where it is cleaved by vacuolar hydrolases into active CPS. However, in the absence of PtdIns $(3,5) \mathrm{P}_{2}$, CPS localizes to the vacuolar membrane instead of the vacuolar lumen. This suggests that CPS on late endosomal membrane failed to be sequestered into ILV before fusion with the lysosome $(86,89)$. Therefore, failure to translocate CPS into the vacuolar lumen in the absence of $\operatorname{Ptd} \operatorname{Ins}(3,5) \mathrm{P}_{2}$ suggests a role for $\operatorname{PtdIns}(3,5) \mathrm{P}_{2}$ in $\operatorname{ILV}$ formation on late endosomes. Although the majority of $\operatorname{Ptd} \operatorname{Ins}(3,5) \mathrm{P}_{2}$ is found on the lysosomal/vacuolar membrane, there is a small population found on late endosomes. This small subset of late endosomal PtdIns(3,5) $\mathrm{P}_{2}$ interacts with Ent3, for the inclusion of cargos into ILVs. Ent3 contains an ENTH (Epsin $\mathrm{NH}_{2}$-Terminal Homology) domain that specifically binds to PtdIns $(3,5) \mathrm{P}_{2}$ on late endosomes. ENTH domains are known to contain $\alpha$-helices that can be inserted into membranes and act as a wedge to drive membrane curvature. Therefore, the binding of the ENTH domain to $\operatorname{PtdIns}(3,5) \mathrm{P}_{2}$ on late endosomes may promote membrane curvature leading to membrane invagination to form ILVs (90).

It was previously mentioned that vacuole plays an important role in storage of metabolites. PtdIns $(3,5) \mathrm{P}_{2}$ has been shown to directly interact with ion channels to regulate their functions. For example, there is a direct interaction between $\operatorname{Ptd} \operatorname{Ins}(3,5) \mathrm{P}_{2}$ and TRPML1, a channel that controls the efflux of lysosomal $\mathrm{Ca}^{2+}$. $\mathrm{Ca}^{2+}$ released by TRPML1 may act on $\mathrm{Ca}^{2+}$ sensor proteins such as calmodulin, and in turn induce membrane fusion by SNAREs (40-42). Protein-lipid interaction 
studies demonstrated that PtdIns $(3,5) \mathrm{P}_{2}$ directly interacts with the N-terminal of TRPML1 and this interaction activates the release of vacuolar $\mathrm{Ca}^{2+}$. Cells deficient in PtdIns(3,5) $\mathrm{P}_{2}$ and cells that lack TRPML1 resulted in reduced $\mathrm{Ca}^{2+}$ release, the enlargement of the vacuole/lysosome, and membrane trafficking defect (91-93).

In addition, it was speculated that $\operatorname{PtdIns}(3,5) \mathrm{P}_{2}$ may be involved in the regulation of proper vacuolar acidification. This speculation is based on two main findings, first, $\operatorname{PtdIns}(3,5) \mathrm{P}_{2}$ deficient cells failed to accumulate quinacrine, a fluorescent dye that accumulates in acidic compartments (Figure 1.7) $(82,83,89)$. Quinacrine is a weak base that is thought to readily permeate the lipid bilayer and becomes fluorescent when protonated. The vacuole provides an acidic environment for the protonation of quinacrine but protonated quinacrine is no longer capable of diffusing back into the cytosol. Therefore, because quinacrine readily accumulates and fluoresces in the vacuolar lumen, quinacrine is commonly used as an acidic indicator and a vacuolar marker $(5,83)$. However, the proportionality of fluorescence intensity with either the concentration of vacuolar quinacrine or the level of vacuolar acidity cannot be determined. Therefore, quinacrine can only be used for qualification but not quantification of the vacuolar acidity. Secondly, PtdIns(3,5) $\mathrm{P}_{2}$ is speculated to directly bind Vph1 based on lipid arrays. The binding of PtdIns(3,5) $\mathrm{P}_{2}$ to $\mathrm{Vph} 1$ stabilizes and increases the V-ATPase activity during hyperosmotic shock and glucose deprivation (94). Although based on the above findings, PtdIns(3,5) $\mathrm{P}_{2}$-deficient vacuoles are expected to be more alkaline, the vacuolar $\mathrm{pH}$ was never quantified. In an attempt to quantify the vacuolar $\mathrm{pH}$ in $\operatorname{Ptd} \operatorname{Ins}(3,5) \mathrm{P}_{2}$-deficient yeast, the current dissertation showed a surprising result, that the vacuolar $\mathrm{pH}$ in $\operatorname{PtdIns}(3,5) \mathrm{P}_{2}$-deficient cells remained acidic in basal conditions. 


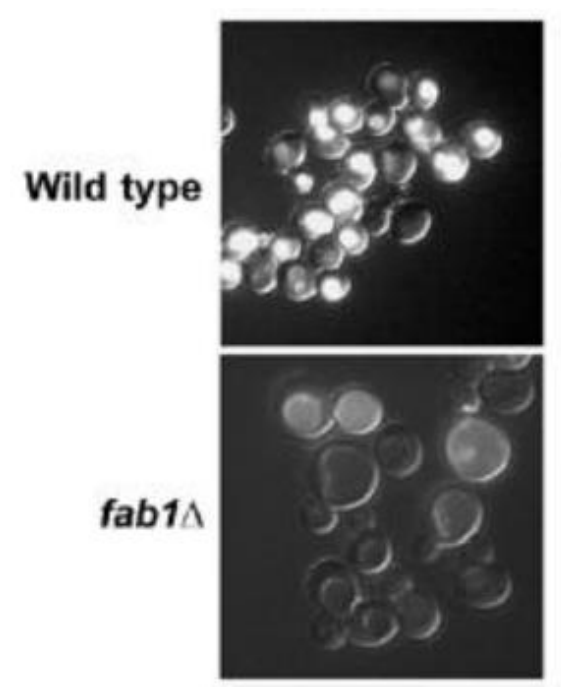

Figure 1. 7 Fab1 is required for the accumulation of quinacrine.

Wild-type yeast accumulated quinacrine in vacuolar compartments (above), while fablऽ yeast deleted for the PtdIns(3)P 5-kinase failed to accumulate quinacrine in enlarged vacuolar structures (bottom) (Image taken with permission from Dove, S. K., Piper, R. C., McEwen, R. K., et al, 2004).

Despite the low level of cellular PtdIns(3,5) $\mathrm{P}_{2}$ compared to other phosphoinositides, PtdIns(3,5) $\mathrm{P}_{2}$ is highly important for proper cellular homeostasis. It is important to decipher how the level of $\operatorname{PtdIns}(3,5) \mathrm{P}_{2}$ is regulated to better understand the function of $\operatorname{PtdIns}(3,5) \mathrm{P}_{2}$. The current players known to be involved in regulation of PtdIns $(3,5) \mathrm{P}_{2}$ include the Fab1/PIKfyve lipid kinase, which phosphorylates the PtdIns(3)P found on endosomal compartments at the 5-hydroxyl position of its inositol ring into PtdIns(3,5) $\mathrm{P}_{2}$ (Figure 1.8). In addition to Fab1, Vac7 in yeast is also a positive regulator that may act upstream of Fab1 to regulate Fab1 activity (Figure 1.8). Deletion of either $F A B 1$ or $V A C 7$ resulted in an undetectable level of PtdIns $(3,5) \mathrm{P}_{2}(95)$. However, the precise mechanism of $\mathrm{Vac} 7$ remains unknown and it is uncertain if there is a Vac7 mammalian homolog. Regulation of $\operatorname{Ptd} \operatorname{Ins}(3,5) \mathrm{P}_{2}$ also requires rapid dephosphorylation of $\operatorname{Ptd} \operatorname{Ins}(3,5) \mathrm{P}_{2}$ into 
PtdIns(3)P by the Fig4/Sac3 lipid phosphatase (Figure 1.8). In addition to Fig4, Atg 18 also acts as

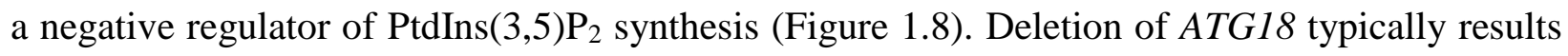
in a 20-fold increase in the level of PtdIns(3,5) $\mathrm{P}_{2}$ (83). Mammalian orthologs of Atg18 are WIPI proteins that are involved in autophagy (96). Interestingly, the synthesis and turnover of PtdIns(3,5) $\mathrm{P}_{2}$ by Fab1 and Fig4, respectively, are closely regulated by Vac14/ArPIKfyve (Figure 1.8). The roles of Fab1, Fig4, and Vac14 in the regulation of $\operatorname{PtdIns}(3,5) \mathrm{P}_{2}$ are further described in detail below.

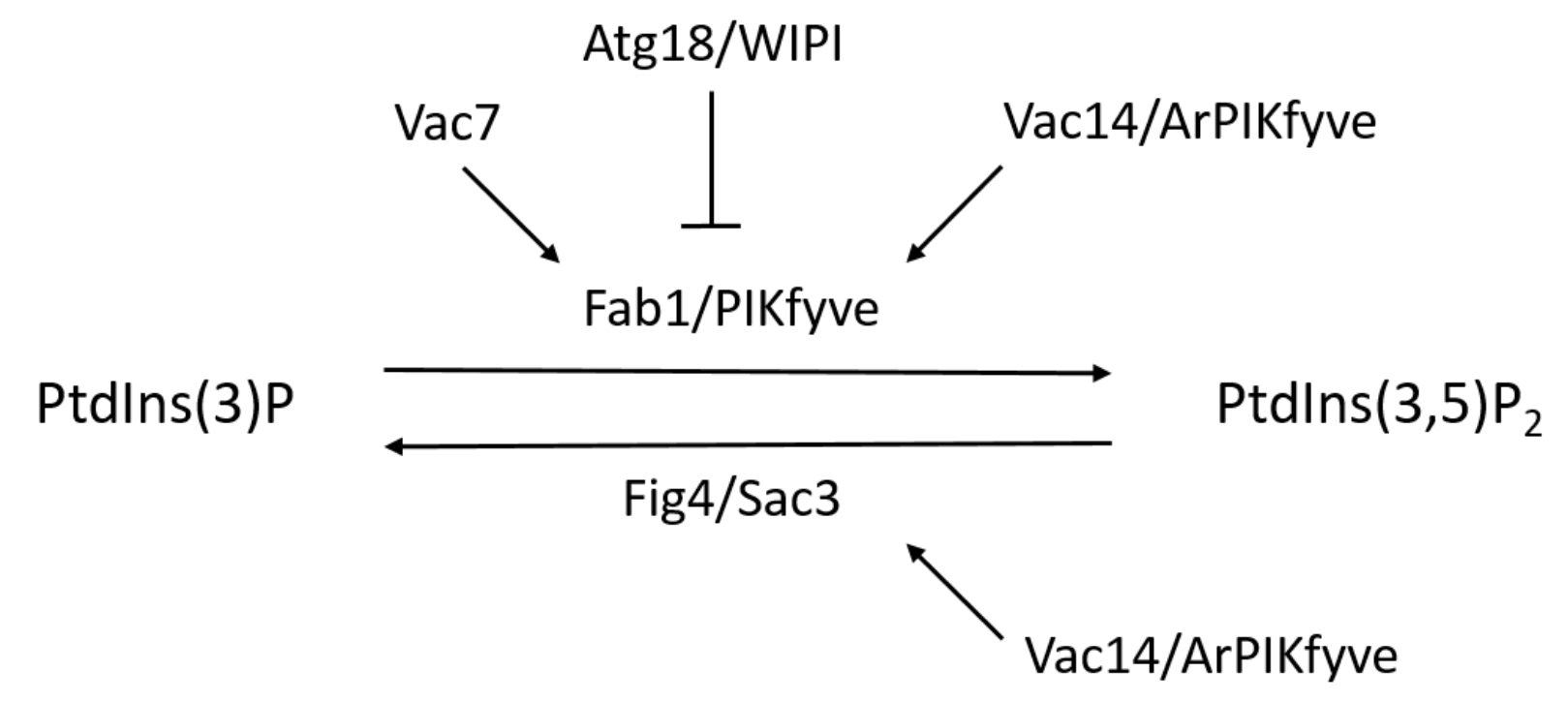

Figure 1. 8 The regulatory pathway of phosphatidylinositol 3,5-bisphosphate.

$\operatorname{PtdIns}(3,5) \mathrm{P}_{2}$ is synthesized by phosphorylating the 5-hydroxl group of PtdIns(3)P by the lipid kinase, Fab1 (or the mammalian homolog, PIKfyve). Vac7 and Vac14 (or the mammalian homolog, ArPIKfyve) function to stimulate and enhance the activity of Fab1/PIKfyve, while Atg18 negatively regulates the synthesis of $\operatorname{Ptd} \operatorname{Ins}(3,5) \mathrm{P}_{2}$. In addition, Vac14/ArPIKfyve also regulates the function of the lipid phosphatase, Fig4 (or the mammalian homolog, Sac3), that dephosphorylates PtdIns(3,5) $\mathrm{P}_{2}$ to generate PtdIns(3)P. 


\subsubsection{The regulation of $\operatorname{PtdIns}(3,5) \mathrm{P}_{2}$ : The Fab1 kinase}

The $F A B 1$ gene was first identified in $S$. cerevisiae from a screen for abnormal nuclear segregation. Deletion of $F A B 1$ resulted in formation of aploid and binucleate cells (97). In addition to the presence of abnormal nuclear segregation, deletion of $F A B 1$ also led to a number of other phenotypes. The most profound phenotype are undetectable levels of $\operatorname{PtdIns}(3,5) \mathrm{P}_{2}$ and gross enlargement of the vacuole (Figure 1.6A,b) (89). The level of $\operatorname{PtdIns}(3,5) \mathrm{P}_{2}$ and vacuolar morphology in $f a b 1 \Delta$ were not rescued in an attempt to stimulate its production by exposure to hyperosmotic stress. This indicates that Fab1 is the main PtdIns(3)P 5-kinase in S. cerevisiae (Figure 1.6A,d) (81). Both inhibition and knockdown of the mammalian ortholog, PIKfyve, resulted in reduced levels of $\operatorname{PtdIns}(3,5) \mathrm{P}_{2}$ and enlarged endolysosomal structures (Figure 1.6B,c) $(1,98)$.

Fab1/PIKfyve contains domains that are evolutionarily conserved: the N-terminal FYVE domain, a middle region GroL-like chaperone domain and the C-terminal catalytic domain. Different from the FYVE domain of EEA1 $\left(\mathrm{FYVE}^{\mathrm{EEA1}}\right)$, the FYVE domain of PIKfyve (FYVE ${ }^{\text {PIKfyve }}$ ) is localized to puncta that are distinguishable from early endocytic structures containing EEA1 (99). FYVE ${ }^{\text {PIKfyve }}$ retains its high affinity for PtdIns(3)P but colocalizes with the late endosome/lysosome markers, LAMP1 (lysosomal-associated membrane protein 1) and the Rab7 GTPase $(70,100)$. Fluorescence studies showed that the EGFP-tagged FYVE domain of PIKfyve (EGFP-FYVE ${ }^{\text {PIKfyve }}$ ) alone localized to the endocytic structures in control COS-7 cells. In COS-7 cells treated with wortmannin, a PtdIns 3-kinase inhibitor to inhibit the synthesis of PtdIns(3)P, EGFP-FYVE ${ }^{\text {PIKfyve }}$ is dispersed in the cytosol (100). Furthermore, PIKfyve ${ }^{\Delta 177-198}$, which lacks the FYVE domain, lost the ability to interact with PtdIns(3)P and remained dispersed in the cytosol $(70,100)$. Studies in yeast also confirm that the FYVE ${ }^{\mathrm{Fab} 1}$ domain is functionally conserved in Fab1. Endogenous GFP-Fab1 localizes to vacuolar membrane and this localization 
is dependent on an intact FYVE domain. Fab1 deleted for the FYVE domain (Fab1 ${ }^{\triangle \mathrm{HINDIII}}$ ) or containing a point mutation in the FYVE domain region $\left(\mathrm{Fab} 1^{\mathrm{FYVE}(\mathrm{c} / \mathrm{s})}\right)$ both resulted in the cytosolic displacement of the corresponding Fab1 mutants (2). Together, these data suggest that the FYVE domain of Fab1/PIKfyve acts to recruit Fab1/PIKfyve to PtdIns(3)P on late endocytic structures.

The C-terminal catalytic domain of PIKfyve has high sequence similarity with the kinase domains found in a number of PtdIns 4-kinases and PtdIns 5-kinases. Expression of the kinasedeficient PIKfyve ${ }^{\mathrm{K} 1831 \mathrm{E}}$, resulted in extensive vacuolation and a decrease in the levels of $\operatorname{PtdIns}(3,5) \mathrm{P}_{2}$ in a number of cell lines (Figure 1.6 B-c) $(1,100)$. Expression of Fab1 containing a point mutation in the catalytic domain also shown a $90 \%$ reduction in the steady-state level of $\operatorname{PtdIns}(3,5) \mathrm{P}_{2}(101)$.

The mid-region of Fab1/PIKfyve contains a GroL-related region that is composed of a CCT and a CCR domain. The GroL domain is also found in the superfamily of chaperones that are known to bind and facilitate proper protein folding (102). This GroL-like region appears to be responsible for Fab1/PIKfyve interaction with its regulators, Fig4 and Vac14 (Figure 1.11). Deletion or point mutations in the CCT or the CCR domains resulted in the loss of interaction with Fig4 and Vac14 (Figure 1.11A). In addition, the level of $\operatorname{Ptd} \operatorname{Ins}(3,5) \mathrm{P}_{2}$ is also reduced to $42 \%$ and $22 \%$ of wild-type levels in cells expressing CCT or CCR point mutants, respectively (2). Together, these data suggest that Fab1/PIKfyve alone is capable of synthesizing $\operatorname{PtdIns}(3,5) \mathrm{P}_{2}$, but optimum synthesis requires interaction with Fig4 and Vac14. 


\subsubsection{The regulation of PtdIns(3,5) $\mathrm{P}_{2}$ : The Fig4 phosphatase}

Fig4, or the mammalian homolog Sac3, contains a conserved N-terminal Sac domain. The Sac domain is known for its phosphoinositide phosphatase activity for the turnover of PtdInsPs. The Sac domain is originally identified in Sac1, a protein involved in actin rearrangements and phospholipid metabolism. The Sac domains found in Sac1, Fig4, and synaptojanin-like proteins contain $\sim 35 \%$ sequence identity and the this catalytic domain alone is able to dephosphorylate PtdIns(3)P, PtdIns(4)P, and PtdIns(3,5) $\mathrm{P}_{2}$ in-vitro (103, 104). However, Sac3 has shown dephosphorylation activity specifically toward $\operatorname{PtdIns}(3,5) \mathrm{P}_{2}$ (105). Interestingly, although recombinant Fig4 showed great specificity in dephosphorylating PtdIns(3,5) $\mathrm{P}_{2}$ into PtdIns(3)P invitro, deletion of FIG4 in yeast did not result in any changes in the level of $\operatorname{PtdIns}(3,5) \mathrm{P}_{2}(106)$. Endogenous Fig4-GFP was shown to co-localize with FM4-64, a vacuolar membrane stain, and this localization is dependent on the C-terminus (Figure 1.9B) (106). The combination of in-vitro phosphatase assay and in-vivo localization assay indicate that Fig4 is a $\operatorname{PtdIns}(3,5) \mathrm{P}_{2}$ 5phosphatase and acts on the vacuolar membrane. However, Fig4 appears to play a more complicated role than just a simple phosphatase.

During hyperosmotic shock, yeast cells initiate a series of complex adaptive response, one aspect of which includes osmotic adjustment by the efflux of intracellular $\mathrm{H}_{2} \mathrm{O}$ from the vacuole into the cytoplasm to compensate for the sudden increase in $\mathrm{NaCl}$ concentration. Accompanying the onset of this adaptive response, the level of PtdIns(3,5) $\mathrm{P}_{2}$ increases dramatically by $\sim 10-20 \%$ of the steady-state level (81-83). The level of PtdIns(3,5) $\mathrm{P}_{2}$ remains the same in fig4 4 cells when exposed to hyperosmotic shock (107). However, when Fig4A cells expressed a catalytically inactive Fig4, the level of PtdIns(3,5)P*2 increased compared to wild-type (2). Therefore, in addition to the turnover of PtdIns(3,5) $\mathrm{P}_{2}$, Fig4 protein seems to also have a role in the synthesis of 
PtdIns(3,5) $\mathrm{P}_{2}$. Mutations in FIG4 have been identified in patients with autosomal recessive Charcot-Marie-Tooth disorder (CMT4J) and an ALS sub-type. The identified mutant alleles of FIG4 show a decrease in the $\operatorname{PtdIns}(3,5) \mathrm{P}_{2}$ level and exhibit enlarged endocytic structures $(3,4)$.

\subsubsection{The regulation of $\operatorname{PtdIns}(3,5) \mathrm{P}_{2}$ : The Vac14 adaptor}

Vac14 was originally identified in a phenotypic screen for mutants that share traits with

$f a b 1 \Delta$ to identify components in the PtdIns(3,5) $\mathrm{P}_{2}$ pathway $(82,108)$. Similar to $f a b 1 \Delta$, vac14 also displays an enlarged vacuolar phenotype, defects in vacuolar segregation, as well as inability to retain quinacrine, a vacuolar acidification indicator $(82,108)$. Sequence comparison indicates that Vac14 is highly conserved in higher eukaryotes (ArPIKfyve). Vac14 is a $\sim 100 \mathrm{kDa}$ protein that contains numerous HEAT-like repeats that are scattered throughout the entire sequence. HEAT (named after Huntingtin, Elongation factor 3, regulatory subunit A of protein phosphatase $2 \mathrm{~A}$, and protein kinase TOR) repeat are known to be a protein-protein interaction motif and is commonly found in membrane-trafficking proteins $(109,110)$. Each HEAT repeat forms two alpha-helices, which when in contact with adjacent HEAT repeats will form a superhelix.

Using both cell extract fractionation and fluorescence microscopy, Vac14 is found to colocalize with Fab1 on the vacuolar membrane (109). Although deletion of Vac14 did not affect

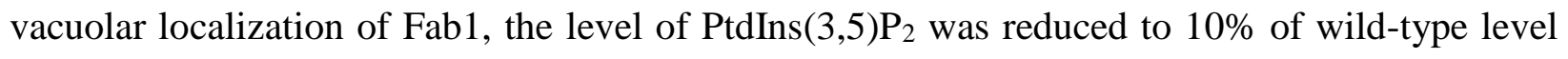
and a concomitant loss of quinacrine accumulation (108). Overexpression of $F A B 1$, compensated for the loss of PtdIns $(3,5) \mathrm{P}_{2}$ in vac144 cells $(82)$. The ability of Fab1 to overcome the deficient PtdIns(3,5) $\mathrm{P}_{2}$ synthesis in vac $14 \Delta$ cells indicates that Vac14 functions upstream of Fab1 to regulate $\operatorname{PtdIns}(3,5) \mathrm{P}_{2}$. The function of Vac14 is conserved in the mammalian homolog, ArPIKfyve. Knockdown of ArPIKfyve using siRNA also resulted in a decrease in the level of $\operatorname{Ptd} \operatorname{Ins}(3,5) \mathrm{P}_{2}$ (105). 
Although sequence analysis predicted that Vac14 does not contain transmembrane or membrane-associating domains, GFP-Vac14 localizes to the vacuolar membrane (109). Therefore, without a transmembrane domain, the vacuolar localization of Vac14 may depend on proteinprotein interaction. Immunoprecipitation (IP) studies demonstrated that there is an interaction between Vac14 and Fab1, and between their respective mammalian counterparts, ArPIKfyve and PIKfyve $(2,105,107,109,111)$. Together with the observation that Vac14 interacts with Fab1, and that vac144 results in a decrease in $\operatorname{Ptd} \operatorname{Ins}(3,5) \mathrm{P}_{2}$ levels, these suggest that Vac14 may interact with Fab1 on the vacuolar/late endosomal membrane to regulate synthesis of $\operatorname{PtdIns}(3,5) \mathrm{P}_{2}$ by Fab1.

In addition to the interaction with Fab1, Vac14 also interacts with Fig4, in both yeast and higher eukaryotes. Fluorescence studies showed that Fig4-GFP is dispersed in the cytosol in vac144 mutant (Figure 1.9D). Interestingly, Vac14-GFP was also largely dispersed in the cytosol in fig4 4 mutant (Figure 1.9C) (106). Together, these suggest that the interaction between Vac14 and Fig4 is required fortheir localization to the vacuolar membrane. Immunoprecipitation and recombinant protein interaction studies in both the yeast and mammalian systems support a direct interaction between Vac14 and Fig4 $(105,106,112)$.

\subsubsection{The regulation of PtdIns $(3,5) \mathrm{P}_{2}$ : The Fab1 complex}

The regulation of PtdIns(3,5) $\mathrm{P}_{2}$ appears to be more complicated than just simply the phosphorylation of PtdIns(3)P by Fab1 and dephosphorylation of PtdIns(3,5) $\mathrm{P}_{2}$ by Fig4. As mentioned above, deletion of Fig4 does not result in an increase in the level of $\operatorname{Ptd} \operatorname{Ins}(3,5) \mathrm{P}_{2}$, while the expression of a catalytically inactive Fig4 in fig $4 \Delta$ resulted in an increase in $\operatorname{Ptd} \operatorname{Ins}(3,5) \mathrm{P}_{2}$ compared to wild-type $(106,107)$. These suggest results thatin addition to its dephosphorylation activity, Fig4 also affects the synthesis of PtdIns $(3,5) \mathrm{P}_{2}$ by Fab1. A series of IP studies indicate that Vac14 is the link between Fig4 and Fab1. First, Vac14 was shown to interact with Fig4 in the 
cytosol, and this interaction does not require Fab1 (Figure 1.10 A and C, respectively) (2). In-vitro interaction assay showed that this Vac14-Fig4 interaction is a direct interaction and does not require additional protein components (Figure 1.11 C) (2). Secondly, Fab1 was shown to only interact and recruit Vac14 and Fig4 to the vacuolar membrane once the cytosolic Vac14-Fig4 complex is formed. This is supported by fluorescence and IP analyses that both Vac14 and Fig4 alone failed to interact and associate with Fab1 in fig4 4 and vac144 mutants, respectively (Figure 1.9C-D; Figure 1.10D-E). IP of Fab1 successfully isolated both Vac14 and Fig4 (Figure 1.10B) (2). In addition, IP analyses indicate that the GroL-related domain of Fab1 alone is enough for interaction with the Vac14-Fig4 complex (Figure 1.11 A-B) (2). Furthermore, as previously mentioned, the level of PtdIns(3,5) $\mathrm{P}_{2}$ also decreased in both fig4 4 and vacl4 4 mutants, indicating that the efficient synthesis of PtdIns(3,5) $\mathrm{P}_{2}$ by Fab1 depends on its interaction with Fig4 and Vac14 $(2,107,108)$. In conclusion, Vac14, Fig4 and Fab1 interact to form a complex (the Fab1 complex), and this complex as a whole regulates both the synthesis and phosphorylation of $\operatorname{PtdIns}(3,5)_{2}$ on the vacuolar membrane. The formation of the Fab1 complex is conserved in mammalian cells, where PIKfyve, ArPIKfyve, and Sac3 also interact to form a complex on the late endolysosomal membrane to regulate $\operatorname{PtdIns}(3,5) \mathrm{P}_{2}(105,112,113)$. 


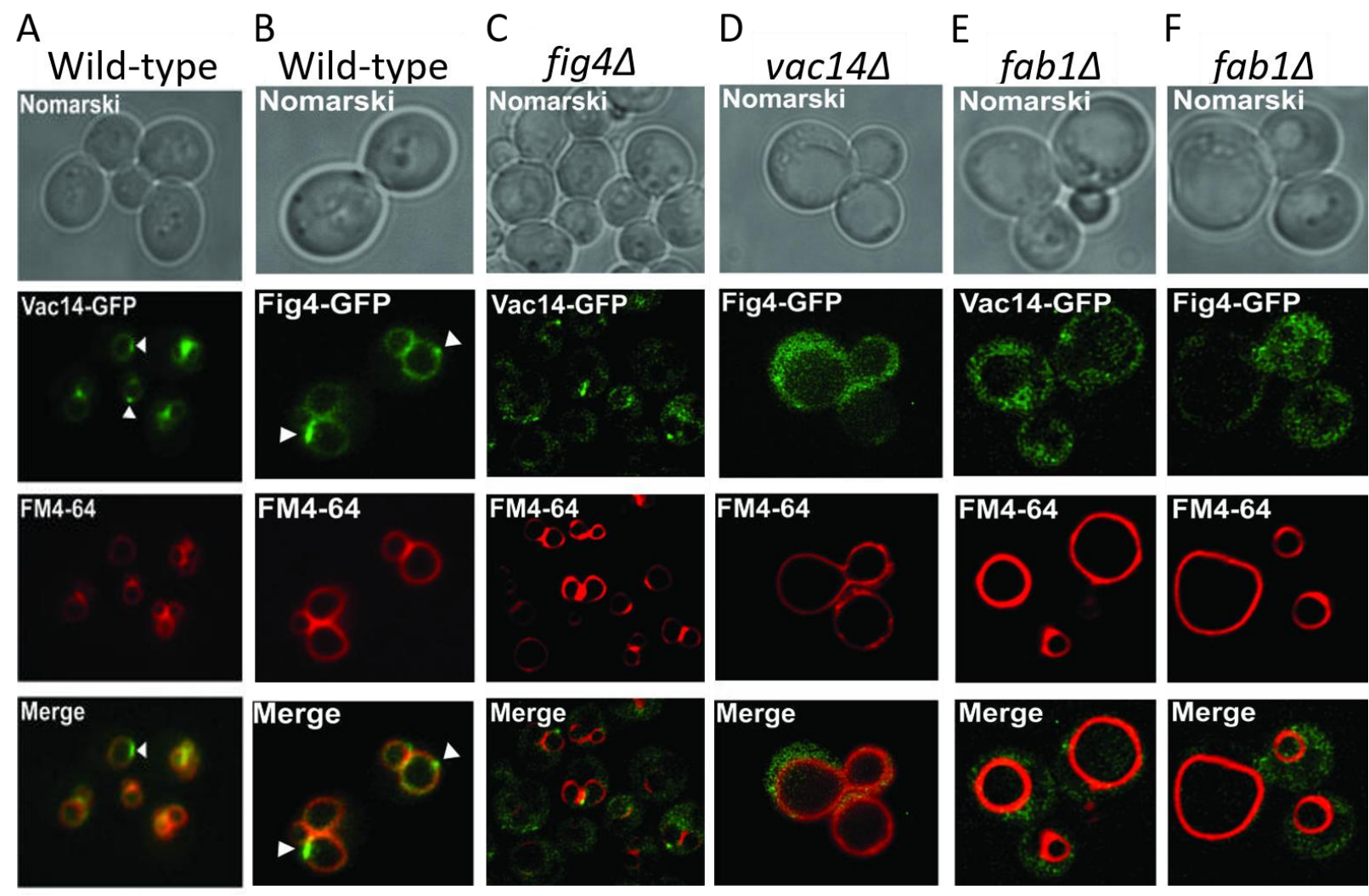

Figure 1. 9 Localization of Vac14 and Fig4 to the vacuolar membrane requires Fab1.

Wild-type, vac14A, fig4A, and fabld cells expressing either Vac14-GFP (A, C, and E, respectively) or Fig4-GFP (B, D, and F, respectively) were labeled with the fluorescent dye FM464 to visualize their localization at the vacuolar limiting membrane (Image modified with permission from Rudge, S. A., Anderson, D. M., and Emr, S. D., 2004). 


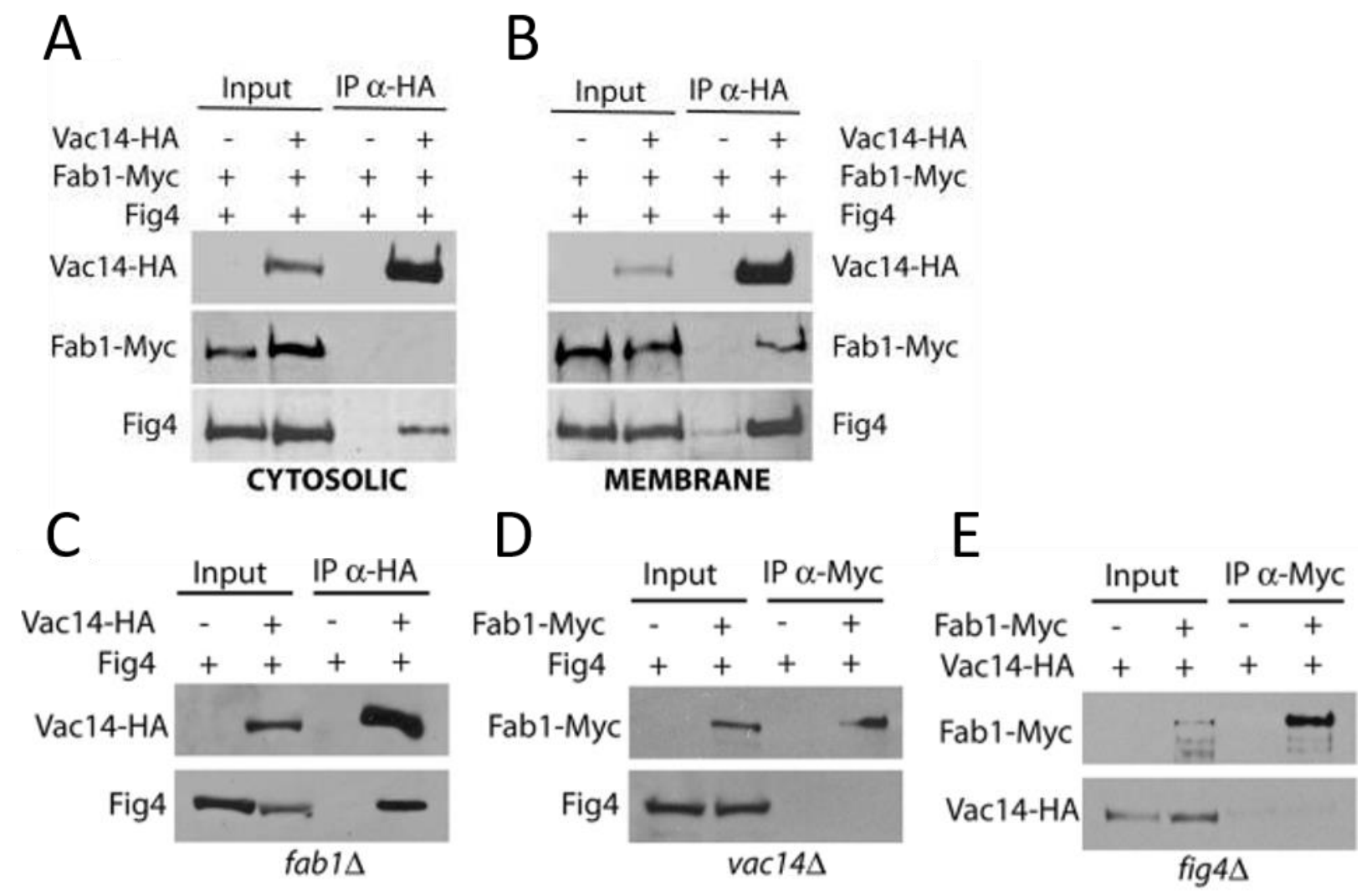

Figure 1. 10 Vac14 interaction with Fig4 is required for association with Fab1.

Immunoprecipitation assays were done using monoclonal anti-HA with the cytosolic fraction (A) and the vacuolar membrane fraction (B) of yeast cells expressing Vac14-HA and Fab1-Myc. (C) Monoclonal anti-HA was used to immunoprecipitate Vac14-HA from whole cell lysates of fab14 mutant. While monoclonal anti-Myc was used to immunoprecipitate Fab1-Myc from whole cell lysates of vac144 (D) and fig44 (E). Detection of Vac14, Fab1, and Fig4 were done using antiHA, anti-Myc, and anti-Fig4 antibodies (Image modified with permission from Botelho, R. J., Efe, J. A., Teis, D., and Emr, S. D., 2008). 


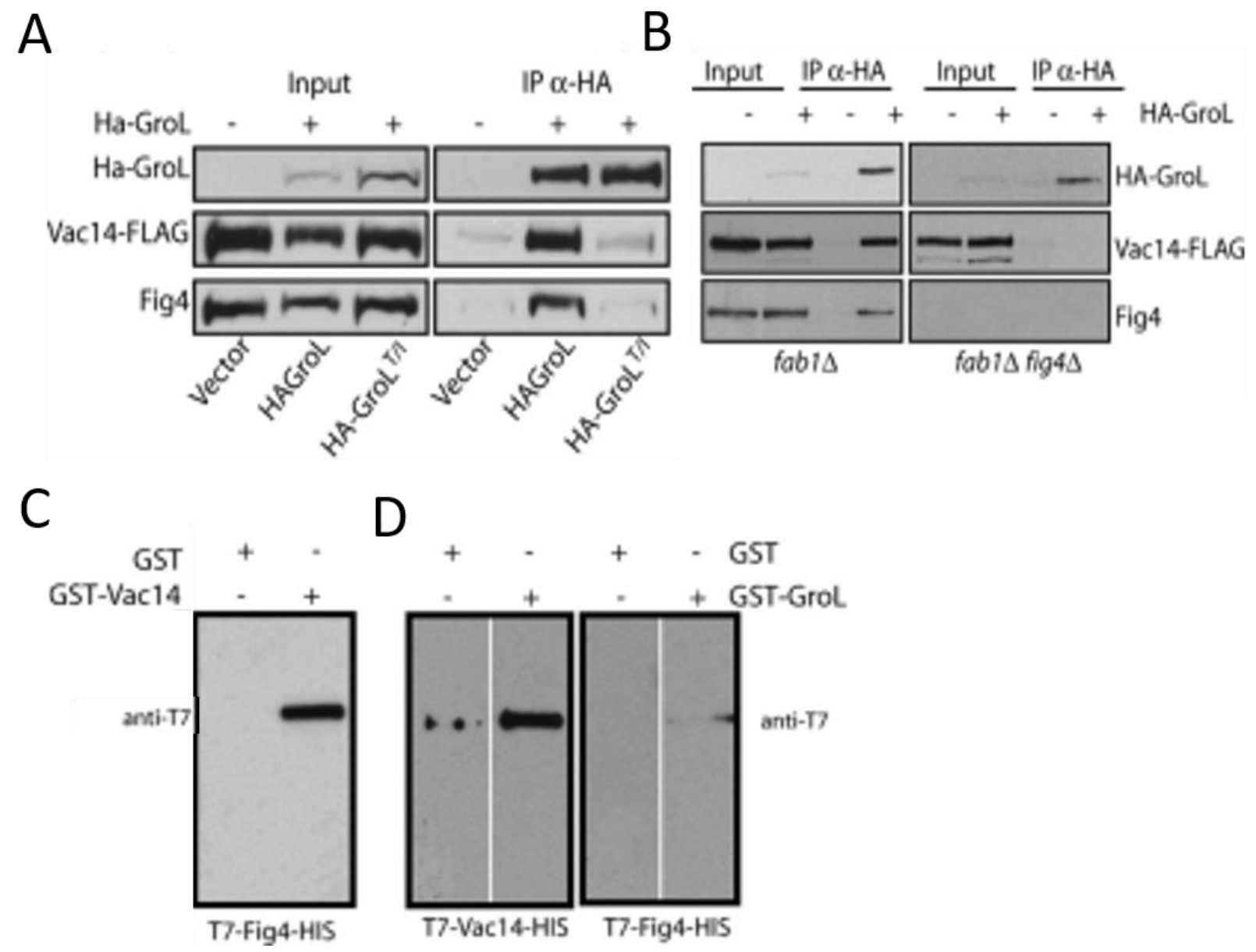

Figure 1. 11 Fab1 interaction with Vac14 and Fig4 requires the GroL-related domain of Fab1. IP with monoclonal anti-HA of whole cell lysates from yeast cells expressing Vac14-FLAG and HA-GroL or the point mutant, HA-GroL ${ }^{\mathrm{T} / \mathrm{I}}$ (A). IP with monoclonal anti-HA of whole cell lysates from fabl $\Delta$ and fabl $\Delta$ fig $4 \Delta$ double mutants (B). In-vitro interaction of purified GST-Vac14 (C) or GST-GroL domain (D) with purified T7-Vac14-HIS and T7-Fig4-HIS (Image modified with permission from Botelho, R. J., Efe, J. A., Teis, D., and Emr, S. D., 2008). 


\subsubsection{The regulation of $\operatorname{PtdIns}(3,5) \mathrm{P}_{2}$ : The Vac14 multimer}

In the midst of identifying the interaction between Vac14, Fig4 and Fab1, Vac14 was found

to be able to interact with at least another molecule of Vac14. When yeast cells were expressing two alleles of Vac14, HA-Vac14 and Vac14-FLAG, IP of HA-Vac14 was able to isolate Vac14FLAG (Figure 1.12B). In addition, in-vitro interaction assay also indicated that recombinant GSTVac14 demonstrated the ability to directly interact with recombinant T7-Vac14-HIS (Figure 1.12A) (2). IP analyses in the mammalian system also support the idea that ArPIKfyve forms homomeric interactions (113). In addition, a number of truncated GFP-tagged ArPIKfyve constructs were used to identify the region responsible for ArPIKfyve homomeric interaction. IP of Myc-ArPIKfyve isolated the wild-type GFP-ArPIKfyve ${ }^{\text {WT }}$ and the GFP-ArPIKfyve (98-782 $^{2}$ (ArPIKfyve containing the C-terminal amino acid 298-782), and GFP-ArPIKfyve ${ }^{\mathrm{Ct}}$ (contains amino acid 523-782)] (Figure 1.12C) (113). There is no interaction between wild-type MycArPIKfyve with the N-terminal of GFP-ArPIKfyve (GFP-ArPIKfyve ${ }^{1-125}$ and GFP-ArPIKfyve ${ }^{1-}$ ${ }^{511}$ ) (Figure 1.12C). Therefore, the C-terminus of ArPIKfyve from amino acid 523 to 782 appears to be critical for homomeric interaction (113).

In an attempt to identify the homomeric state of ArPIKfyve, COS-7 cells expressing MycArPIKfyve were cross-linked with methanol-free formaldehyde reagent before immunoprecipitation of Myc-ArPIKfyve. Immunoblotting analysis of the IP of the cross-linked Myc-ArPIKfyve resulted in three separate protein bands with approximate molecular weight of $82 \mathrm{kDa}, 170 \mathrm{kDa}$, and $250 \mathrm{kDa}(113)$. Based on literature and previous findings, the molecular weight of the monomer ArPIKfyve is $\sim 82 \mathrm{kDa}$, therefore, the IP analysis suggests that ArPIKfyve may interact to form a homodimer $(170 \mathrm{kDa})$ or a homotrimer $(250 \mathrm{kDa})$. However, this approach of crosslinking proteins in-vivo is questionable since it cannot rule out the presence of other potential ArPIKfyve binding partners. 


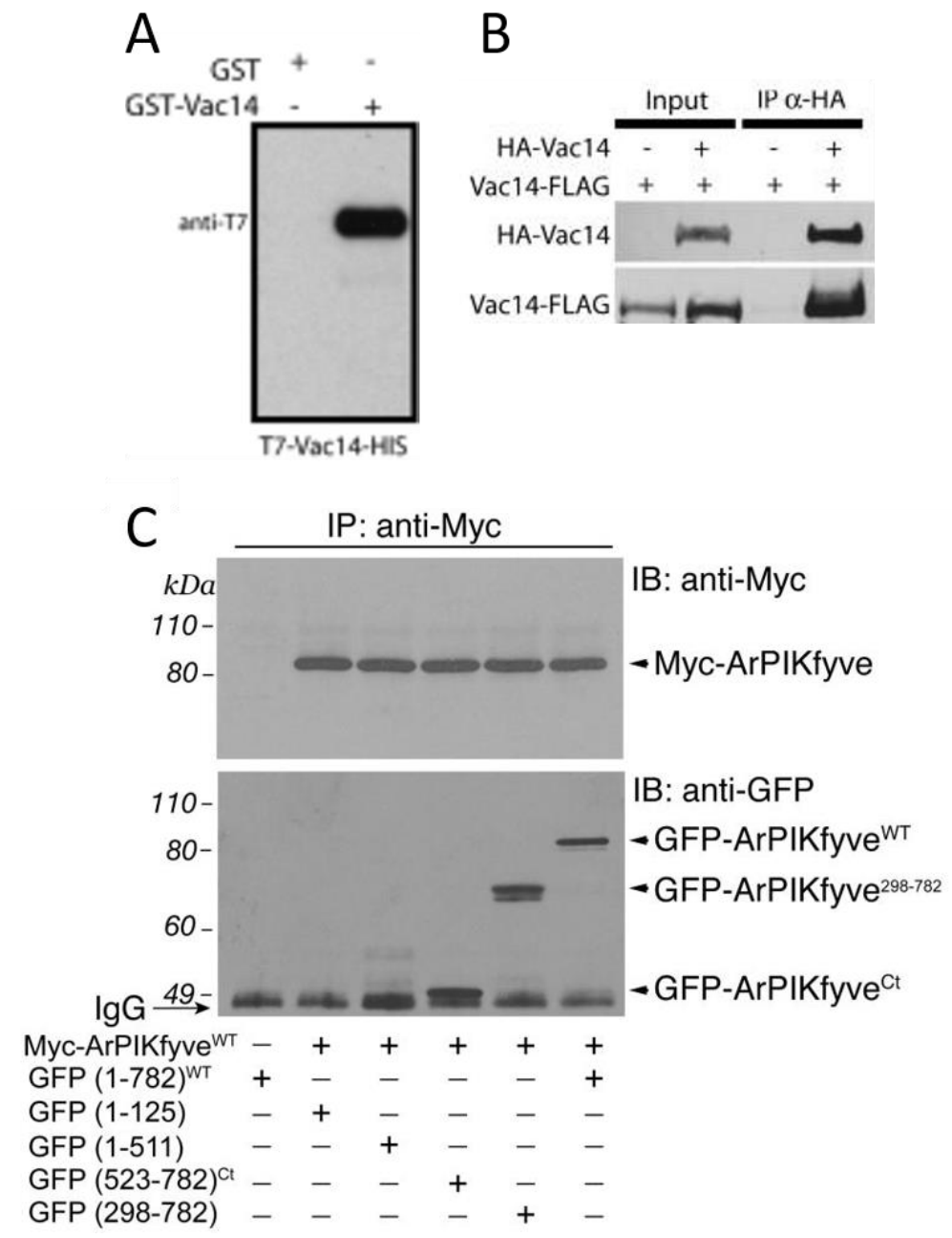

Figure 1. 12 Vac14 homomeric interaction.

(A) In-vitro binding assay of purified GST-Vac14 with purified T7-Vac14-HIS. T7-Vac14-HIS is detected with monoclonal anti-T7 (from 2). (B) IP with monoclonal anti-HA of whole cell lysates from yeast cells expressing HA-Vac14 and Vac14-FLAG (Image modified with permission from Botelho, R. J., Efe, J. A., Teis, D., and Emr, S. D., 2008). (C) IP with anti-Myc of COS-7 cells expressing Myc-ArPIKfyve and a combination of GFP-tagged ArPIKfyve constructs containing the indicated amino acid region. The GFP-ArPIKfyve mutants were detected with an anti-GFP antibody (Image modified with permission from Sbrissa, D., Ikonomov, O. C., Fenner, H., and Shisheva, A., 2008). 


\subsection{Rationale and hypothesis}

1. Based on the literature, both the yeast Vac14 and the mammalian ArPIKfyve can interact with another copy of itself to form a homomeric complex. This homomeric complex was suggested to be either a homodimer or a homotrimer based on in-vivo crosslinking immunoprecipitation analysis. However, the functional relevance of this homomeric interaction is not known. Therefore, we hypothesize that similar to the mammalian model, the yeast Vac14 also interacts to form a homomeric complex (Vac14 multimer) that forms an essential core for the assembly of the Fab1 complex and is required for the regulation of $\operatorname{PtdIns}(3,5) P_{2}$.

2. Quinacrine is a fluorescent dye that accumulates in the vacuoles and is commonly used as an acidic marker for the vacuole. Yeast cells deficient in $\operatorname{Ptd} \operatorname{Ins}(3,5) \mathrm{P}_{2}$ (ie. fabl $1 \Delta$ and vac144) resulted in the loss of vacuolar quinacrine, which suggest that vacuoles in cells deficient in PtdIns(3,5) $\mathrm{P}_{2}$ are not acidified properly. However, quinacrine is only qualitative, and thus the vacuolar $\mathrm{pH}$ of $\operatorname{Ptd} \operatorname{Ins}(3,5) \mathrm{P}_{2}$-deficient yeast is still unknown. In addition, PtdIns $(3,5) \mathrm{P}_{2}$ was shown to interact with $\mathrm{Vph} 1$, a subunit of the V-ATPase, and may function to help stabilize the formation and function of V-ATPase during hyperosmotic stress and glucose-deprivation. We hypothesize that the lack of PtdIns(3,5) $P_{2}$ may result in an alkaline vacuolar $p H$ due to the loss of a functional $V$ ATPase that may require PtdIns(3,5)P $P_{2}$ for assembly and function. 


\subsection{Objectives}

To address hypothesis 1 , the following goals were set:

(1) Confirm Vac14 homomeric interaction region using Vac14 truncation constructs in-vivo

(2) Confirm that the C-terminal motif of Vac14 is required for direct Vac14 homomerization in-vitro

(3) Characterize the homomeric state of recombinant Vac14

(4) Elucidate the importance of Vac14 homomerization for the regulation of PtdIns(3,5)P2

The work is published in the Journal of Biological Chemistry in 2013, and co-authorship is shared with T. Alghamdi (84).

To address hypothesis 2 , the following goals were set:

(1) Determine the relative vacuolar acidity in $\operatorname{PtdIns}(3,5) \mathrm{P}_{2}$-deficient yeast cells with pHluorin, a $\mathrm{pH}$-sensitive variant of green fluorescence protein (GFP)

(2) Design and quantitatively measure vacuolar $\mathrm{pH}$ of $\operatorname{PtdIns}(3,5) \mathrm{P}_{2}$-deficient yeast using the pH-sensitive fluorescent dye, 5-(and 6-) carboxy-2',7'-dichlorofluorescein diacetate (cDCFDA).

(3) Identify the role of PtdIns(3,5) $\mathrm{P}_{2}$ in vacuolar acidification during hyperosmotic stress

(4) Quantify the lysosomal $\mathrm{pH}$ in the mammalian cells deficient in $\operatorname{Ptd} \operatorname{Ins}(3,5) \mathrm{P}_{2}$

This work is published in the Journal of Biological Chemistry in 2015 (114). 
Chapter 2 Materials and Methods 


\subsection{Yeast and bacterial strains}

DH5 $\alpha$ (Invitrogen) and BL21 DE3 (Stratagene) bacteria were grown in selective LB media and used for cloning of plasmid DNA and recombinant protein expression, respectively. All yeast strains weregrown in either YPD medium or selective synthetic-deficient (SD) medium. The Saccharomyces cerevisiae strains used in this study are listed in Table 1.

\subsection{Plasmids and cloning}

All plasmids used in this study are listed in Table 2. The yeast Vac14 truncations and point mutants were previously generated by Amra Saric, Danielle Taylor, and Tamadher Alghamdi. The full-length VAC14 in S. cerevisiae was used as a template, the Vac14 truncations and point mutants were PCR amplified and inserted into a yeast expression plasmid containing a FLAG epitope (pRB415A-FLAG). T7-Vac14-His used in the recombinant study was previously characterized (2). The full-length VAC14 in S. cerevisiae was PCR amplified and inserted into pET23d(+). The Vac14 truncations and point mutants were also cloned and inserted into the pET23d(+) vector for recombinant protein expression. The full-length VAC14 was also cloned into pETDuet-1 by inserting VAC14 between the 5'EcoRI and 3'XhoI restriction sites and used for expression of recombinant Vac14-S·tag.

\subsection{Generation of deletion strains and yeast transformation}

The PCR-based gene deletion method was used to generate vph14 in the SEY6210 background (115). Briefly, the Phusion high-fidelity DNA polymerase (New England BioLabs) along with the F1 and R1 primer plasmid-specific sequences were used to generate DNA for $v p h 1 \triangle$. The PCR product was transformed into SEY6210 using the lithium acetate method to allow for homologous recombination. Successful homologous recombination was confirmed by PCR.

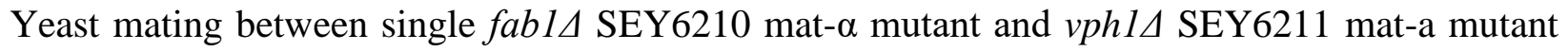


were used to generate $f a b 1 \Delta v p h 1 \Delta$ double mutant. The fab1 1 Mup1-pHluorin and vph1 $\Delta$ Mup1-

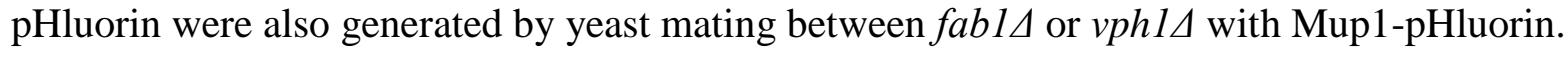

\subsection{Recombinant protein expression and purification}

Plasmid DNA was transformed into BL21 DE3 using heat shock method and plated on Luria Broth (LB) plates containing $100 \mu \mathrm{g} / \mathrm{ml}$ ampicillin and incubated at $37^{\circ} \mathrm{C}$ overnight. The transformant was inoculated into Super Broth (SB) medium containing $100 \mu \mathrm{g} / \mathrm{ml}$ ampicillin and grown at $37^{\circ} \mathrm{C}$ overnight with constant agitation. The overnight culture was inoculated into SB with $100 \mu \mathrm{g} / \mathrm{ml}$ ampicillin to an optical density (OD) of 0.15 , and grown overnight at $16^{\circ} \mathrm{C}$ for recombinant protein expression. Culture was pelleted and lysed with $0.5 \mathrm{mg} / \mathrm{ml}$ lysozyme for 2 hours at $4^{\circ} \mathrm{C}$ in lysis buffer (50 mM Tris- $\mathrm{HCl}, \mathrm{pH} 8.0,300 \mathrm{mM} \mathrm{NaCl}$, and $20 \mathrm{mM}$ imidazole) supplemented with $30 \mathrm{mg} / \mathrm{ml}$ EDTA, $2 \mathrm{mM} \beta$-mercaptoethanol (BME), $0.2 \mathrm{mM}$ AEBSF, and bacterial protease inhibitor cocktail A (Bio-basic Ltd.). Cells were further lysed by sonication in an ice bath for 4 pulses of $15 \mathrm{sec}$ (output 50). Supernatant was collected and incubated with $\mathrm{Ni}^{2+}$ NTA beads (Bio-basic Ltd.) or S-protein agarose (Novagen) for 2 hours at $4{ }^{\circ} \mathrm{C}$ with constant rotation for purification of His-tagged or S.tag recombinant proteins, respectively. Beads were collected and washed with lysis buffer. His-tagged recombinant proteins were eluted with elution buffer (50 mM Tris- $\mathrm{HCl}, \mathrm{pH} 8.0,300 \mathrm{mM} \mathrm{NaCl}$, and $250 \mathrm{mM}$ imidazole) supplemented with 2 $\mathrm{mM} \mathrm{BME}$ at $4{ }^{\circ} \mathrm{C}$ with constant rotation. Eluted recombinant proteins were dialyzed overnight in D-Tube Dialyzer Maxi (Novagen) at $4^{\circ} \mathrm{C}$ in dialysis buffer $(50 \mathrm{mM}$ Tris- $\mathrm{HCl}, \mathrm{pH}$ 8.0, $200 \mathrm{mM}$ $\mathrm{NaCl}$ ) supplemented with $2 \mathrm{mM} \mathrm{BME}$. Purified recombinant proteins were stored at $-80^{\circ} \mathrm{C}$ for invitro protein binding assay. 


\subsection{In-vitro protein binding assay}

S-protein agarose coated with S.tag recombinant proteins was incubated with purified recombinant T7-Vac14-His 6 in the presence of $0.05 \%$ Tween 20 , at $4^{\circ} \mathrm{C}$ for 2 hours with constant rotation. Beads were washed and recombinant proteins were eluted with 2x sample buffer $(100 \mathrm{mM}$ Tris, pH 6.8, 4\% SDS, $10 \%$ glycerol, $1 \%$ bromophenol blue, and 10\% BME)

\subsection{Fast protein liquid chromatography}

A Sephacryl S300 column (GE Healthcare) and the AKTA FPLC system were used to characterize the molecular weight of recombinant proteins. Fractionations were performed at $4{ }^{\circ} \mathrm{C}$ with a flow rate of $0.250 \mathrm{ml} / \mathrm{min}$ in dialysis buffer. Recombinant proteins and $300 \mu \mathrm{g}$ of protein standards - phosphorylase (Sigma), catalase (Sigma), ferritin (Calbiochem), thyroglobulin (Sigma), and blue dextran (Sigma) - were injected individually onto the FPLC. Recombinant protein elutions were collected in $2 \mathrm{ml}$ fractions. Samples were concentrated with $10 \%$ Trichloroacetic acid (TCA) and processed for Western blotting.

\subsection{Differential velocity ultracentrifugation with glycerol gradient}

Recombinant proteins and protein standards (phosphorylase b, catalase, ferritin, and thyroglobulin) were loaded on top of individual 10 to $40 \%$ glycerol solutions in dialysis buffer prepared in $11 \mathrm{ml}$ polyallomer tubes. Proteins were separated by spinning at 30,000 rpm for 4 hours at $4^{\circ} \mathrm{C}$ by a swinging bucket SW41 Ti rotor (Beckman Coulter) with a floor-model ultracentrifuge (Optima L-100K, Beckman Coulter). The presence of protein standards was detected by reading absorbance at $280 \mathrm{~nm}\left(\mathrm{~A}_{280}\right)$. Recombinant proteins were collected in $1 \mathrm{ml}$ fractions and processed with 10\% TCA for Western blotting. 


\subsection{Co-immunoprecipitation}

Yeast cultures grown to OD of $\sim 0.6$ were lysed with 15 units of zymolyase in spheroplasting medium (2\% glucose, $1 \mathrm{X}$ amino acids, $1 \mathrm{M}$ sorbitol, $20 \mathrm{mM}$ Tris, $\mathrm{pH} 7.5$, and $1 \mathrm{X}$ yeast nitrogen base) for $30 \mathrm{~min}$ at $30^{\circ} \mathrm{C}$ with constant agitation. Spheroplasted cells were washed and further lysed in a $2 \mathrm{ml}$ Dounce homogenizer in $1 \mathrm{ml}$ HEPES-lysis buffer (20 mM HEPES, pH 7.2, $50 \mathrm{mM}$ potassium acetate, $200 \mathrm{mM}$ sorbitol, and $2 \mathrm{mM}$ EDTA), supplemented with $3 \mathrm{X}$ complete protease inhibitor mixture (Biobasic) and $0.3 \mathrm{mM}$ 4-(2-aminoethyl) benzene sulfonyl fluoride (AEBSF). Lysates were solubilized in $0.25 \%$ Tween 20 and incubated with $1 \mu \mathrm{g} / \mathrm{ml}$ monoclonal anti-FLAG antibodies (M2; Sigma-Aldrich) for 1 hour at $4^{\circ} \mathrm{C}$. Lysates were further incubated with $200 \mu 1$ of GammaBind G-linked Sepharose beads (GE Healthcare) for 2 hours at $4^{\circ} \mathrm{C}$. Beads were collected and washed with $0.25 \%$ Tween 20 in HEPES-lysis buffer. Bound proteins were eluted with 2x sample buffer and used for Western blotting.

\subsection{SDS-PAGE and Western blotting}

Protein samples in $2 \mathrm{x}$ sample buffer were heated at $100^{\circ} \mathrm{C}$ for $5 \mathrm{~min}$ before loaded onto discontinuous polyacrylamide gel with 5\% stacking and 9\% separating gels. All SDS-PAGEs were run at 130 Volts for 2 hours in 1x Tris/SDS running buffer (Bio-basic Ltd.). Proteins separated by SDS-PAGE were transferred to a polyvinylidene fluoride (PVDF) membrane (Pall Corporation) at 100 Volts for 1 hour in 1x Tris/Glycine transfer buffer (Bio-basic Itd.). Membranes were blocked for 1 hour at room temperature in 1x Tris-buffered saline (TBS) $(20 \mathrm{mM}$ Tris, $150 \mathrm{mM} \mathrm{NaCl}, 2$ $\mathrm{mM} \mathrm{KCl}, \mathrm{pH} 7.4$ ) supplemented with $0.05 \%$ Tween-20 and 5\% skim milk. Membranes were then incubated with corresponding primary antibody in TBS with $0.05 \%$ Tween-20 and 5\% skim milk at $4{ }^{\circ} \mathrm{C}$ overnight with constant agitation. Membranes were washed with TBS and incubated with the appropriate secondary antibody at $4^{\circ} \mathrm{C}$. Washed membranes were exposed to Immuno-Star WesternC chemiluminescence reagents (Bio-Rad) and analyzed using Gel-Doc System (Bio-Rad). 
Monoclonal anti-T7 antibodies (EMD Millipore) were used at 1:15,000 dilution. Monoclonal anti-FLAG antibodies (M2; Sigma-Aldrich) were used at 1:2,500 dilution. Monoclonal anti-HA antibodies (HA.C5; Abcam) were used at 1:2000 dilution. Polyclonal antiFig4 antibodies were used at 1:20,000 dilution (106). Monoclonal anti-S $\cdot$ tag antibodies were used a 1:5000 (EMD Millipore). Horseradish peroxidase (HRP)-linked secondary anti-mouse antibodies were used at 1:5,000 dilution. HRP-linked secondary anti-rabbit antibodies were used at 1:25,000 dilution. ImmunoStar Western C chemiluminescence reagents (Bio-Rad) and the GelDoc System (Bio-Rad) were used to exposed membranes.

\subsection{Vacuolar staining, hyperosmotic shock, and fluorescence microscopy}

FM4-64 labeling- Yeast cells were incubated with $15 \mu$ M FM4-64 (Invitrogen) for 20 min at $30^{\circ} \mathrm{C}$. Cells were washed and incubated in selective SD medium for 1 hour at $30^{\circ} \mathrm{C}$. Quinacrine labeling-Cells were incubated in 100 mM HEPES-KOH, pH7.6 with $200 \mu \mathrm{M}$ quinacrine for 10 min at $30^{\circ} \mathrm{C}$. Cells were washed and re-suspended in $100 \mathrm{mM}$ HEPES-KOH, pH7.6 with $2 \%$ glucose for imaging.

CMAC labeling - Cells were incubated in selective SD medium with $100 \mu \mathrm{M}$ CMAC in the dark for $15 \mathrm{~min}$ at room temperature. Cells were washed and re-suspended in selective SD medium for imaging.

cDCFDA labeling - Cells were incubated in synthetic complete (SC) medium, $\mathrm{pH}$ 7.5, with $50 \mu \mathrm{M}$ cDCFDA for 1 hour at $26^{\circ} \mathrm{C}$. Cells were washed and re-suspended in SC medium, $\mathrm{pH} 7.5$, for fluorimetry and imaging. Hyperosmotic shock - after labeling, cells were exposed to $0.9 \mathrm{M}$ $\mathrm{NaCl}$ for $10 \mathrm{~min}$ at $30^{\circ} \mathrm{C}$. Cells were washed and re-suspended in selective SD medium for fluorimetry or imaging. 


\subsection{Fluorimetry}

A series of standard $\mathrm{pH}$ buffers $(\sim \mathrm{pH} 4.5-7.5)$ was prepared with 50mM MES, $50 \mathrm{mM}$ Hepes, $50 \mathrm{mM} \mathrm{KCl}, 50 \mathrm{mM} \mathrm{NaCl}, 0 . \mathrm{M}$ ammonium acetate, $10 \mathrm{mM} \mathrm{NaN}_{3}$, and $10 \mathrm{mM} 2$ deoxyglucose. Yeast cells labeled with cDCFDA were pooled in $10 \mathrm{ml}$ of SC media, $\mathrm{pH}$ 7.5. For V-ATPase activity inhibition, cDCFDA-labeled cells were treated with $1 \mu \mathrm{M}$ ConA for 1 hour before incubating in standard $\mathrm{pH}$ buffers for fluorescence measurements. Two-hundred microliters of cells were mixed with $1 \mathrm{ml}$ of respective standard $\mathrm{pH}$ buffers supplemented with $50 \mu \mathrm{M}$ CCCP for $4 \mathrm{~min}$. The resulting solutions were immediately transferred into quartz cells for fluorescence spectra measurement using a fluorimeter (Hitachi F-2500) with excitation scan between 400$520 \mathrm{~nm}$ and emission at $535 \mathrm{~nm}$. Two-hundred microliters of cells mixed with $1 \mathrm{ml}$ of standard $\mathrm{pH}$ buffers without CCCP were also measured to obtain basal fluorescence intensity. Samples were measured in triplicate and the average fluorescence intensity for the respective $\mathrm{pH}$ values were fitted to a sigmoidal-dose response curve using the GraphPad Prism 6 software.

\subsection{Cell culture and lysosomal labeling}

RAW264.7 macrophages were cultured in DMEM (Dulbecco's modified Eagle's medium)

supplemented with $10 \% \mathrm{FBS}$ (fetal bovine serum) at $37^{\circ} \mathrm{C}$ with $5 \% \mathrm{CO}_{2}$. Cells were passaged and plated on glass slides to grow to $50-70 \%$ confluence. Cells were incubated with $2 \mathrm{mg} / \mathrm{ml} \mathrm{FITC-}$ dextran for an hour and chased for an hour in DMEM at $37^{\circ} \mathrm{C}$ with $5 \% \mathrm{CO}_{2}$. Before imaging, cells were treated with either DMSO, $20 \mathrm{nM}$ apilimod, $200 \mathrm{nM} \mathrm{MF4}$, or $1 \mu \mathrm{M}$ conA for an hour. Afterward, cells were washed with PBS and imaged by ratiometric imaging. 


\subsection{Ratiometric imaging}

Cells coated on a glass slide were incubated in calibration media $(145 \mathrm{mM} \mathrm{KCl}, 10 \mathrm{mM}$

glucose, $1 \mathrm{mM} \mathrm{MgCl} 2$, and $20 \mathrm{mM}$ Hepes, $\mathrm{pH}$ 7.2) and imaged in a Leiden environmental chamber maintained at $37^{\circ} \mathrm{C}$. Cells were sequentially incubated in different $\mathrm{pH}$ buffers $(7.5,6.5,5.5$, and 4.5) supplemented with $10 \mu \mathrm{M}$ nigericin and $5 \mu \mathrm{M}$ monensin. At each $\mathrm{pH}$, cells were incubated for $5 \mathrm{~min}$ and images were taken with excitation at $485 \mathrm{~nm}$ and $438 \mathrm{~nm}$ before subsequent change in $\mathrm{pH}$ buffer. The fluorescence ratios of wild-type and swollen lysosomes were selected at random over three independent experiments. The measured values for the respective $\mathrm{pH}$ values of the calibration buffer were fitted to a sigmoidal-dose response curve using the GraphPad Prism 6 software. 


\section{Chapter 3 Results}

Chapter 3.1: This work is published in the Journal of Biological Chemistry in 2013, and coauthorship is shared with T. Alghamdi (84).

Chapter 3.2: This work is published in the Journal of Biological Chemistry in 2015 (114). 


\subsection{Characterization and the importance of homomeric Vac14}

Immunoprecipitation analyses of different ArPIKfyve truncation constructs suggest that its

C-terminal 523-782 amino acid region is sufficient for ArPIKfyve binding (113). To investigate the conservation of the binding region in Vac14, yeast vac14 truncation constructs and point mutants in the Vac14 C-terminus were generated by my colleague Tamadher Alghamdi (116). IP studies were used to confirm the binding ability of the C-terminus of Vac14 and also the loss of Vac14 interaction in the C-terminus point mutants (116).

\subsubsection{Co-immunoprecipitation to show Vac14 self-interaction in-vivo using Vac14 truncations}

Co-immunoprecipitation assay is often used for identifying protein-protein interaction invivo. First, to confirm Vac14 self-interaction exists in-vivo, yeast expressing Vac14-HA was transformed with wild-type Vac14-FLAG. If Vac14 self-interacts, Vac14-HA will interact with Vac14-FLAG to form a complex. By isolating Vac14-FLAG using anti-FLAG antibodies and Protein G-linked beads, Vac14-HA bound to Vac14-FLAG will also be isolated. The presence of isolated proteins was confirmed by Western blotting and detection using anti-FLAG and anti-HA antibodies. As expected based on literature, immunoprecipitation of Vac14-FLAG recovered Vac14-HA, indicating the presence of Vac14 homomerization in-vivo (Figure 3.1). As a negative control, cells expressing only Vac14-HA were also subjected to co-immunoprecipitation by antiFLAG antibodies. This is to verify that anti-FLAG antibodies and Protein G-linked beads do not form non-specific interaction with Vac14-HA (Figure 3.1). Next, to confirm the Vac14 C-terminus alone is sufficient for Vac14 self-interaction in-vivo, yeast expressing Vac14-HA was transformed with either $v a c 14^{41-350}$-FLAG or $v a c 14^{41-500}$-FLAG, mutants containing portions of the Cterminus. FLAG-tagged Vac14 mutants were immunoprecipitated from cell lysates using antiFLAG antibodies. As expected, based on preliminary results from T. Alghamdi, 
immunoprecipitation of $V a c 14^{41-350}$-FLAG and Vac1 $4^{41-500}$-FLAG successfully co-purified Vac14-HA (Figure 3.1). The recovery of Vac14-HA by Vac14 mutants containing only the Cterminus indicates that the Vac14 N-terminus is not necessary for Vac14 self-interaction (Figure 3.1). To further define the region responsible for Vac14 self-interaction, T. Alghamdi generated point mutants in the C-terminus of Vac14 based on conserved motifs across different species (Figure 3.2). Using co-immunoprecipitation assays, T. Alghamdi confirmed that there was a 5090\% loss of Vac14-HA recovery by the Vac14 point mutants (116). 


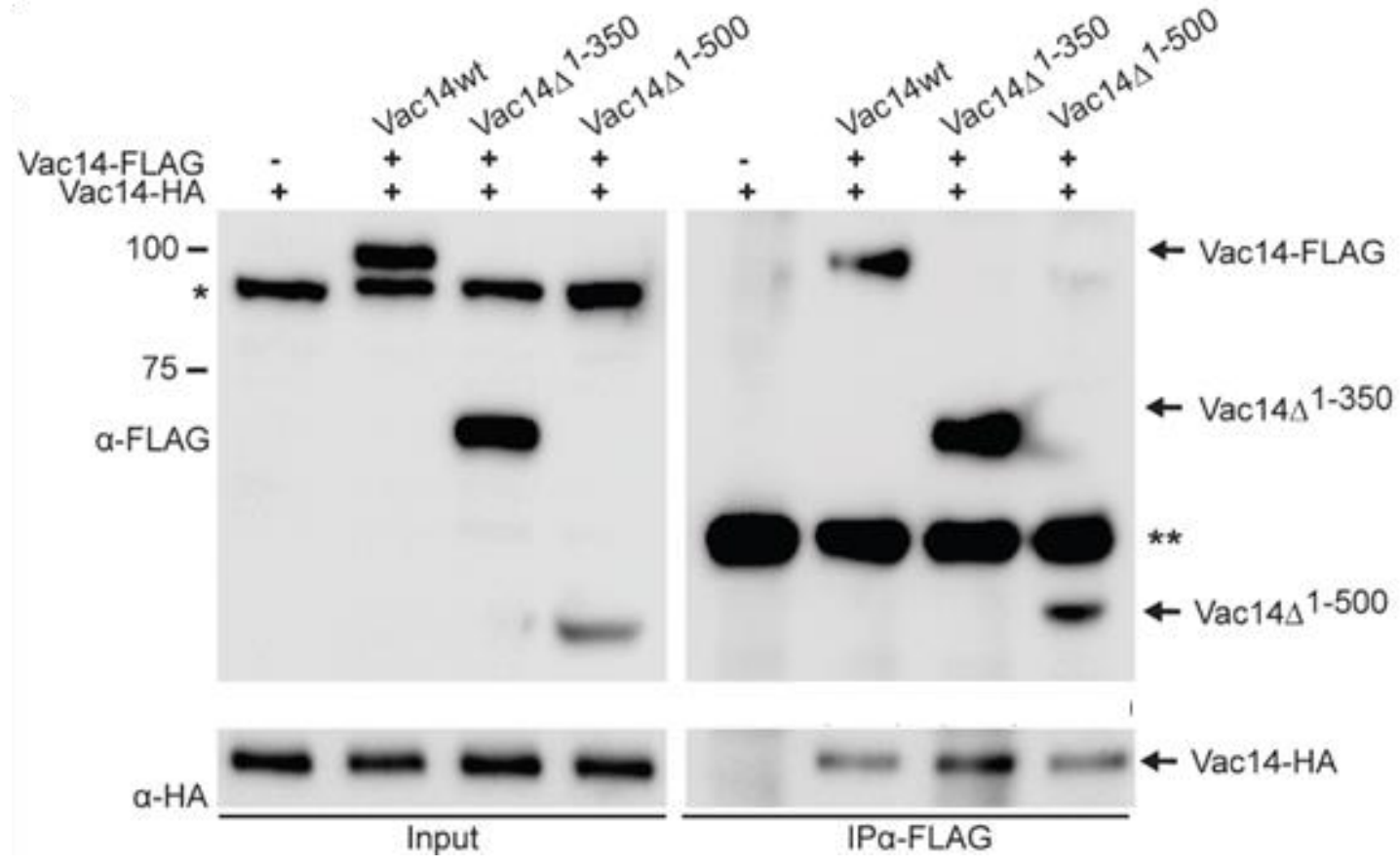

Figure 3. 1 Vac14 C-terminus alone is necessary for Vac14 self-interaction in yeast.

Yeast expressing Vac14-HA was transformed with either empty plasmid (-), Vac14 ${ }^{\mathrm{WT}}$-FLAG, vac14 $4^{41-350}$-FLAG, or vac14 $4^{41-500}$-FLAG. Immunoprecipitation was performed using monoclonal anti-FLAG antibodies. SDS-PAGE and Western blot were used to separate and probe for presence of wild-type Vac14 and Vac14 point mutants using anti-FLAG and anti-HA antibodies. (*) indicates non-specific bands. (**) indicates antibody heavy chain. 


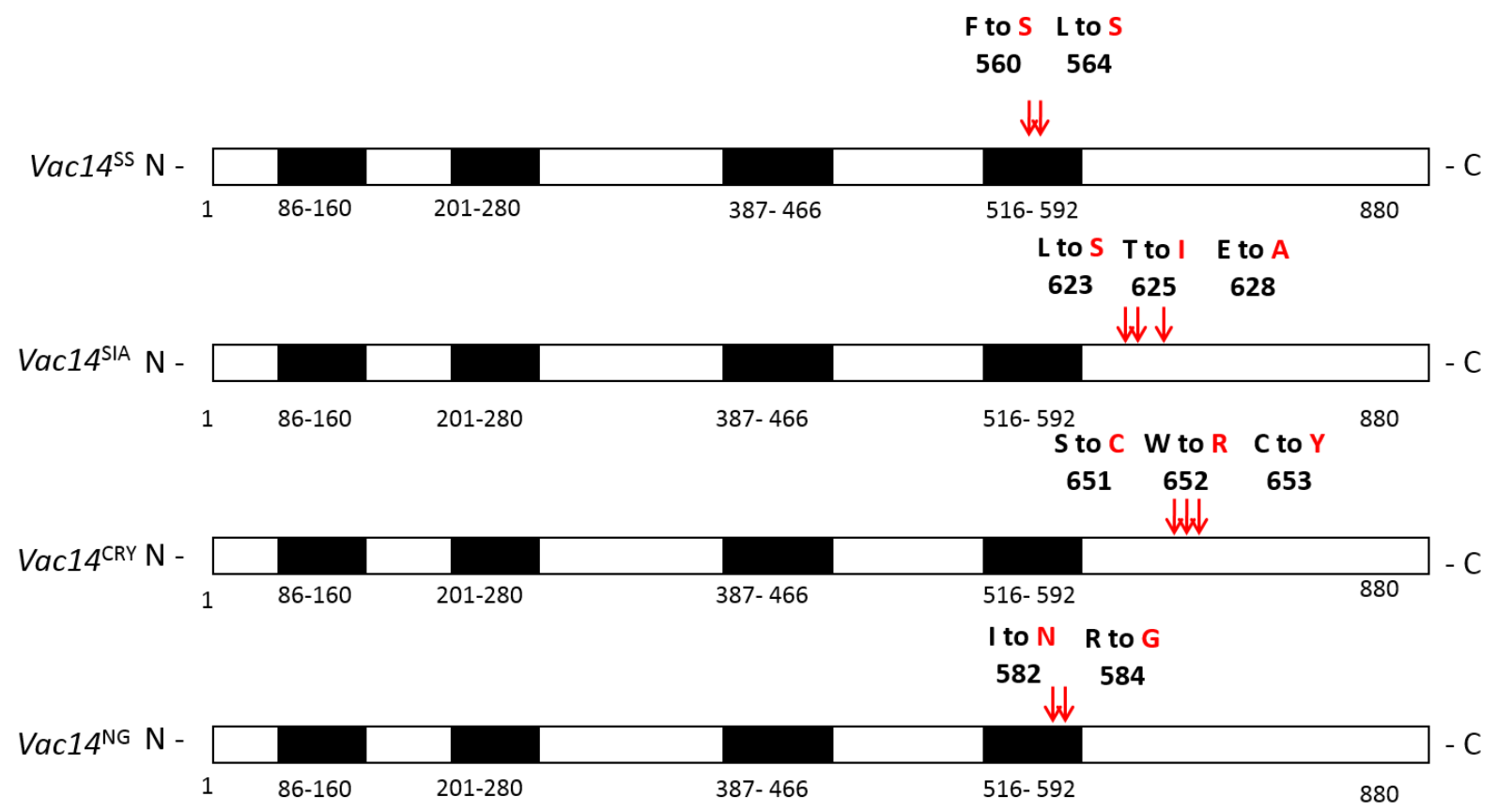

Figure 3. 2 Diagram illustrating the location of point mutations on Vac14.

Amino acids selected for point mutations based on conserved residues identified through multiple alignments of Vac14 (arrows). The mutated amino acids (red) and their specific location are indicated. Representation of HEAT repeats (black bars). 


\subsection{2 in-vitro recombinant Vac14 interaction with Vac14 truncations and point mutants}

To confirm that the results from the co-IP studies are the result of direct Vac14 selfinteraction, recombinant Vac14 truncations were generated for in-vitro interaction studies. Vac14 truncations containing only the $\mathrm{N}$-terminus (Vac14 ${ }^{\Delta 557-880}$ ) and only the C-terminus (Vac14 ${ }^{\Delta 1-334}$ ) were generated and fused to a T7- and His 6 -tag in the $\mathrm{N}$ - and C-terminus, respectively. Vac14 fulllength fused to S•tag was also generated to test for Vac14 self-interaction. Vac14 full-length and truncations were first expressed in E.coli BL21(DE3) and purified separately using $\mathrm{Ni}^{2+}{ }_{-} \mathrm{NTA}$ or S-protein agarose affinity chromatography. Purified T7-Vac14-HIS 6 were then incubated with Vac14-S•tag coated S-protein agarose to allow for Vac14 self-interaction. Both purified T7Vac14-HIS 6 and T7-Vac14 ${ }^{\Delta 557-880}{ }_{-H I S}$ were incubated with S-protein agarose to show that there is no non-specific interaction between purified recombinant proteins and S-protein agarose (Figure 3.3A). As expected, both full-length T7-Vac14-HIS 6 and the Vac14 C-terminus (T7-Vac14 ${ }^{\Delta 1-334}$ ) were recovered by Vac14-S•tag, while the Vac14 N-terminus (T7-Vac14 ${ }^{\Delta 557-880}$ ) was not (Figure 3.3zA). In addition, recombinant Vac14 point mutants fused to T7- and $\mathrm{HIS}_{6}$-tag were also generated (by T. Alghamdi) and tested for in-vitro interaction with Vac14-S•tag (Figure 3.3B; 3.3z). Surprisingly, all the recombinant Vac14 point mutants tested were not recovered from Vac14-S•tag affinity chromatography (Figure 3.3B). Together, both in-vitro and in-vivo studies suggest that the mutated regions in the Vac14 C-terminus prevented Vac14 self-interaction. The Vac14 point mutants represent monomeric Vac14 that have lost the ability to self-interact. Purified recombinant T7-Vac14 ${ }^{\mathrm{SS}}$-His 6 was selected for further analysis of the homomeric state of Vac14. 


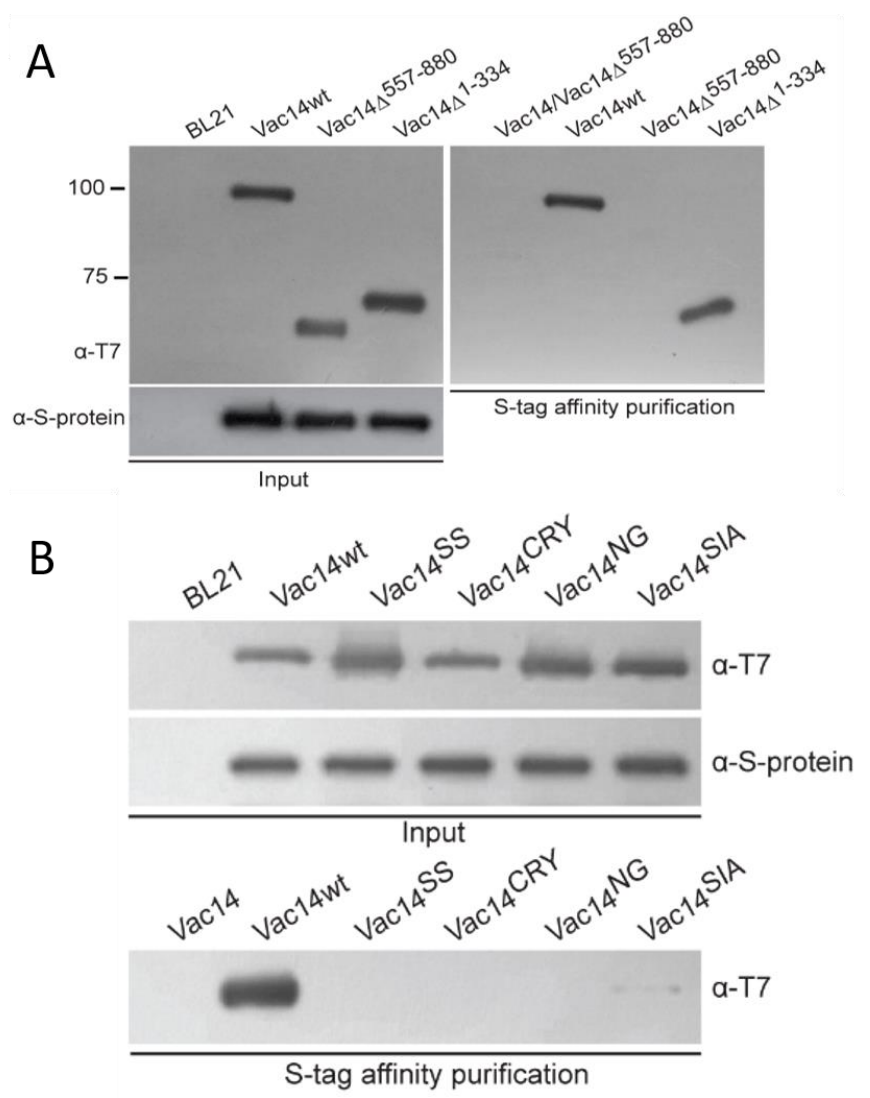

Figure 3. 3 Vac14 C-terminus mutants disrupted Vac14 self-interaction in-vitro.

(A) Recombinant Vac14 wild-type and truncations containing N-terminal T7 and C-terminal His6 epitopes (Vac14 ${ }^{\mathrm{wt}}, \mathrm{Vac14}{ }^{\Delta 557-880}$, and $\mathrm{Vac} 14^{\Delta 1-334}$ ) were purified using $\mathrm{Ni}^{2+}$-NTA batch affinity chromatography. Purified T7-Vac14 wild-type and truncations were incubated with purified Vac14 ${ }^{\mathrm{wt}}$-S $\bullet$ tag coated S-protein agarose. Recombinant protein inputs (left) and bound recombinant Vac14 (right) were analyzed by Western blotting and detected using monoclonal $\alpha$-T7 and $\alpha$-Sprotein antibodies. (B) Purified recombinant Vac14 wild-type and point mutants containing Nterminus T7 and C-terminus His6 epitopes (Vac14 ${ }^{\mathrm{wt}}, \mathrm{Vac} 14^{\mathrm{SS}}, \mathrm{Vac} 14^{\mathrm{CRY}}$, Vac14 ${ }^{\mathrm{NG}}$, and Vac14 ${ }^{\mathrm{SIA}}$ ) were incubated with purified Vac $14^{\mathrm{wt}}$-S $\bullet$ tag coated S-protein agarose. Recombinant protein inputs (top) and bound recombinant Vac14 (bottom) were analyzed by Western blotting and detected using monoclonal $\alpha$-T7 and $\alpha$-S-protein antibodies. 


\subsubsection{Characterization of recombinant Vac14 multimer by fast protein liquid chromatography}

Purified recombinant $\mathrm{T} 7-\mathrm{Vac} 14^{\mathrm{wt}}-\mathrm{HIS}{ }_{6}$ and $\mathrm{T} 7-\mathrm{Vac} 14^{\mathrm{SS}}$-His6 were used to identify the homomeric state of Vac14 by size-exclusion chromatography. To characterize the Vac14 homomeric state, first, a mixture of protein standards with different molecular weights were used to identify their fractionation pattern in a Sephacryl S300 size-exclusion column. Proteins are separated based on their Stoke's radius or shape and molecular weight, with heavier and irregularly shaped proteins eluting first, while smaller and globular proteins migrate through the column much slower and elute later in the fractions. Eluents were measured at an absorbance of $280 \mathrm{~nm}$ for the presence of proteins by an online ÄKTA FPLC system. As expected, the protein standards were fractionated according to their molecular weight starting with blue dextran $(\sim 2,000 \mathrm{kDa})$ eluted first in the void volume, followed by thyroglobulin $(\sim 660 \mathrm{kDa})$, ferritin $(\sim 440 \mathrm{kDa})$, catalase $(\sim 250 \mathrm{kDa})$, and phosphorylase b ( $\sim 97 \mathrm{kDa})$ (Figure 3.4A). Due to the low quantity of purified recombinant proteins, recombinant Vac14 eluents were collected in $1 \mathrm{ml}$ fractions and tested for the presence of T7-Vac14-HIS 6 and T7-Vac14 ${ }^{\mathrm{SS}^{-}} \mathrm{HIS}_{6}$ by Western blotting with anti-T7 antibodies. The majority of recombinant $\mathrm{T} 7-\mathrm{Vac} 14^{\mathrm{SS}}$-HIS 6 eluted in fractions that correspond to ferritin, which indicates that the monomeric Vac14 migrates as a protein with molecular weight of $\sim 400 \mathrm{kDa}$ (Figure 3.4B, bottom). The majority of T7-Vac14-HIS 6 homomeric complex eluted in the void volume that corresponds to blue dextran (Figure 3.4B, top). A minority of T7-Vac14-HIS 6 showed a migration pattern similar to the monomeric T7-Vac14 ${ }^{\mathrm{SS}}-\mathrm{HIS}_{6}$. Although the monomeric Vac14 has a molecular weight of $100 \mathrm{kDa}$, it had a migration pattern that resembled a protein with a molecular weight of $\sim 440 \mathrm{kDa}$. This suggests that Vac14 has a large Stoke's radius, and therefore does not migrate as a globular protein through the size-exclusion column. Furthermore, because 
Vac14 appears to have a large Stoke's radius, the multimeric Vac14 complex was unable to be resolved by the Sephacryl S300 column and eluted in the void volume (Figure 3.4B).

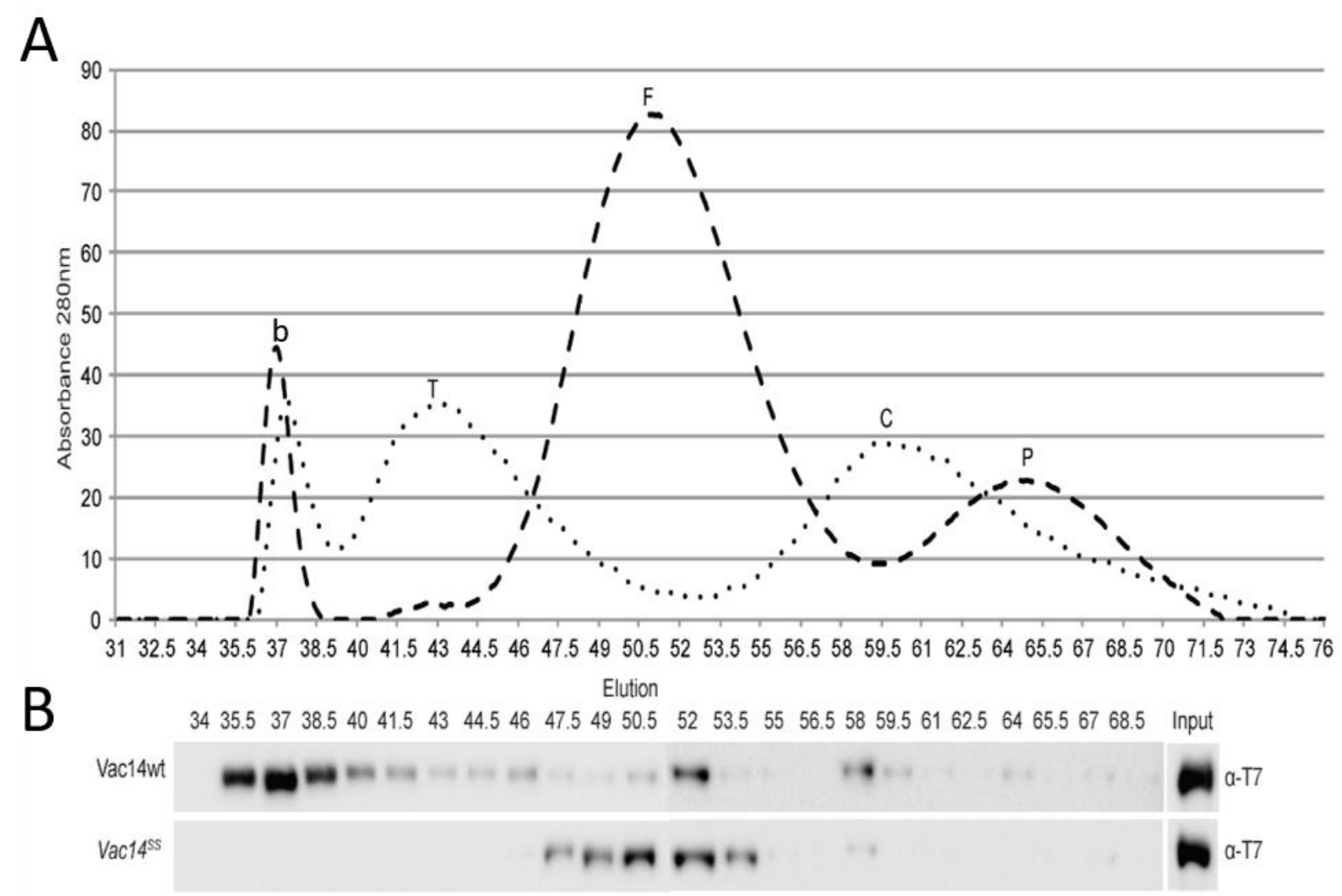

Figure 3. 4 FPLC migration pattern of homomeric and monomeric recombinant Vac14.

(A) Protein standards: blue dextran (b), thyroglobulin (T), ferritin (F), catalase (C), and phosphorylase b (P) were eluted at $1 \mathrm{ml} / \mathrm{min}$ from the Sephacryl S300 using the ÄKTA FPLC system. Elution of protein standards was detected simultaneously by absorbance reading at $280 \mathrm{~nm}$. (B) Purified T7-Vac14-HIS 6 (top) and $\mathrm{T} 7-\mathrm{Vac} 14^{\mathrm{SS}^{-}} \mathrm{HIS}_{6}$ (bottom) were eluted at $1 \mathrm{ml} / \mathrm{min}$ and collected in $1 \mathrm{ml}$ fractions. The presence of recombinant proteins in each fraction was detected by Western blotting using $\alpha$-T7 antibody. Input represents $10 \%$ of the total corresponding recombinant protein injected onto the Sephacryl S300. 


\subsubsection{Characterization of recombinant Vac14 multimer by differential velocity ultracentrifugation}

Differential velocity ultracentrifugation with glycerol gradient was used to further identify the homomeric state of recombinant Vac14. The migration of proteins through a glycerol gradient is mainly based on molecular weight and is less affected by the Stoke's radius compared to FPLC. Protein standards were used to identify the fractionation pattern of proteins with different molecular weight through a 10-40\% glycerol gradient. After velocity ultracentrifugation, $1 \mathrm{ml}$ fractions were collected and measured for the presence of proteins at A280nm. Heavier proteins migrate further through the glycerol gradient, while smaller proteins have a shorter migration distance. The majority of phosphorylase $\mathrm{b}(\sim 97 \mathrm{kDa})$ was found in fraction 1 , followed by catalase $(\sim 250 \mathrm{kDa})$ in fraction 2 , ferritin $(\sim 440 \mathrm{kDa})$ in fraction 5 and thyroglobulin $(\sim 660 \mathrm{kDa})$ in fraction 9 (Figure 3.5A). Fractions containing recombinant Vac14 were detected by Western blotting with anti-T7 antibodies. The majority of the monomeric Vac14, T7-Vac14 ${ }^{\mathrm{SS}}-\mathrm{HIS}_{6}$, was found in fraction 2 and has a migration peak that corresponds to catalase with a molecular weight of $\sim 250 \mathrm{kDa}$ (Figure 3.5B, bottom). However, the majority of the monomeric Vac14 did not fractionate in fraction $1(\sim 97 \mathrm{kDa})$ suggesting that the migration of proteins through the glycerol gradient was still affected by Stoke's radius but less significantly as compared to migration through FPLC. The T7-Vac14 ${ }^{\mathrm{wt}}-\mathrm{HIS}_{6}$ multimer complex has a migration peak between fractions 2 and 3, which suggests that $\mathrm{T} 7-\mathrm{Vac}_{1} 4^{\mathrm{wt}}$-HIS ${ }_{6}$ migrates as a protein complex with a molecular weight $>250 \mathrm{kDa}$ (Figure 3.5B, top). The slight shift in migration pattern of monomeric and multimeric Vac14 suggests that Vac14 homomeric complex likely exists as a homodimer. 


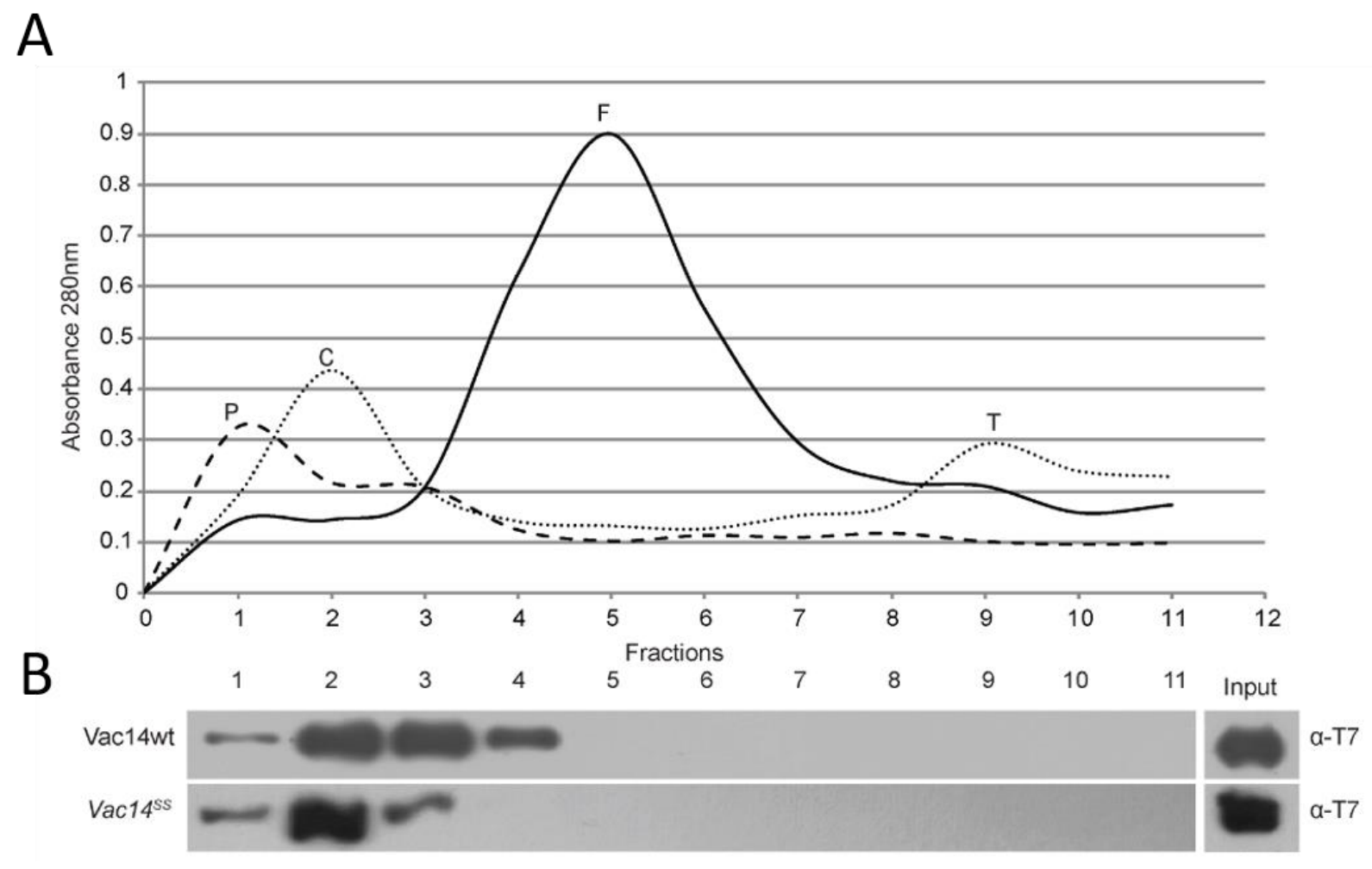

Figure 3. 5 The migration pattern of homomeric and monomeric recombinant Vac14 in differential velocity ultracentrifugation with 10-40\% glycerol gradient.

A set of $10-40 \%$ glycerol gradients were prepared in separate $11 \mathrm{ml}$ polyallomer round bottom tubes. Purified recombinant proteins (T7-Vac14-HIS 6 and $\mathrm{T} 7-\mathrm{Vac}_{6} 4^{\mathrm{SS}}-\mathrm{HIS}_{6}$ ) and protein standards: phosphorylase $b(\mathrm{P})$, catalase $(\mathrm{C})$, ferritin $(\mathrm{F})$ and thyroglobulin $(\mathrm{T})$ were each layered onto individual glycerol gradients and centrifuged at 30,000 rpm for 5 hours. Glycerol gradients were then collected in $1 \mathrm{ml}$ fractions for (A) detection of protein standards at A280nm (B) detection of recombinant proteins with Western blotting using $\alpha-\mathrm{T} 7$ antibody. Input represents $10 \%$ of total corresponding recombinant protein layered onto the glycerol gradient. 


\subsubsection{Co-Immunoprecipitation to show Vac14 self-interaction is required for interaction with Fig4}

Vac14 is known to interact with Fig4 to form a complex and is suggested to be required for the localization of Vac14 -Fig4 cytosolic complex to the vacuolar membrane. To investigate the importance of Vac14 multimer in the formation of the Vac14-Fig4 complex, co-IP was used to test the ability to purify Fig4 with point mutants, Vac1 $4^{S S}$-FLAG and Vac1 $14^{C R Y}$-FLAG. As expected, immunoprecipitation of Vac14 ${ }^{\mathrm{WT}}$-FLAG co-recovered Fig4, which confirms that Vac14 interacts with Fig4 to form a protein complex (Figure 3.6). However, immunoprecipitation of monomeric $\mathrm{Vac}_{1} 4^{S S}$-FLAG and Vac14 ${ }^{C R Y}$-FLAG failed to recover Fig4 (Figure 3.6). The inability of monomeric Vac14 to purify Fig4 suggests that the formation of a Vac14 homodimer is necessary for its interaction with Fig4.

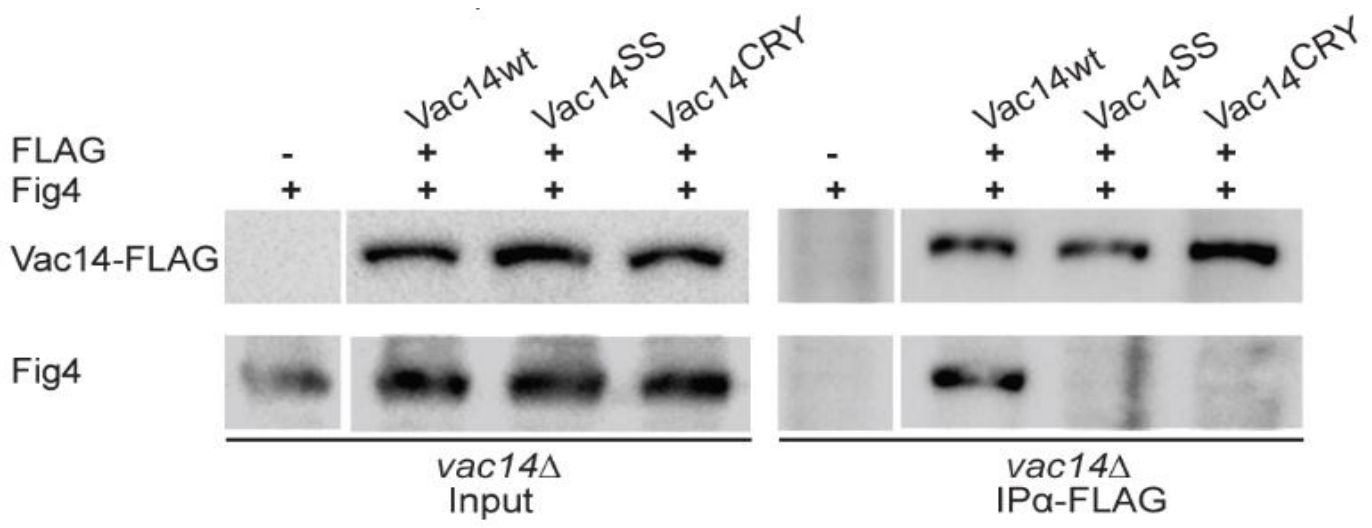

Figure 3. 6 The Vac14 homomeric complex is required for Vac14 interaction with Fig4.

Yeast vac144 mutant was transformed with FLAG-tagged Vac14 (Vac14 ${ }^{\mathrm{wt}}$, vac14 ${ }^{S S}$, or vac14 ${ }^{C R Y}$ ) and immunoprecipitated using monoclonal $\alpha$-FLAG antibody. Western blotting with $\alpha$-FLAG and anti-Fig4 antibody were used to detect for the presence of Vac14 and the recovery of Fig4, respectively. 


\subsubsection{Fluorescence microscopy to show Vac14 self-interaction is required for the rescue of vacuolar morphology}

To further understand the importance of the Vac14 homomeric complex, vac144 cells were transformed with monomeric Vac14 point mutants (vac14 $4^{S S}$ and $v a c 14^{C R Y}$ ). Cells deficient in PtdIns(3,5) $\mathrm{P}_{2}$, such as the vac14 mutant which has only $\sim 10 \% \operatorname{PtdIns}(3,5) \mathrm{P}_{2}$, exhibit a single enlarged vacuole as compared to small multi-lobed vacuoles in wild-type cells (108). The ability of monomeric Vac14 point mutants to rescue the vacuolar enlargement due to lack of $\operatorname{Ptd} \operatorname{Ins}(3,5) \mathrm{P}_{2}$ indicates the importance of Vac14 multimer in the synthesis of $\operatorname{PtdIns}(3,5) \mathrm{P}_{2}$. Vacuolar morphology can be observed using fluorescence microscopy by labeling vacuolar membrane with the red fluorescent dye, FM4-64. The expression of wild-type VAC14 rescued the enlarged vacuolar morphology while expression of monomeric $\operatorname{Vac} 14^{S S}$ and $\mathrm{Vacl} 4^{N G}$ failed to rescue the defective vacuolar phenotype (Figure 3.7A).

Yeast cells under hyperosmotic condition are known to exhibit a dramatic increase in $\operatorname{PtdIns}(3,5) \mathrm{P}_{2}$ and fission of the vacuoles. Increase in $\operatorname{Ptd} \operatorname{Ins}(3,5) \mathrm{P}_{2}$ may potentially serve to control vacuolar size to regulate the decrease in vacuolar volume due to the expulsion of water during hyperosmotic condition. Yeast vac144 mutant expressing either wild-type Vac14, or Vac14 point mutants were used to identify the role of Vac14 multimer in the regulation of PtdIns $(3,5)_{2}$ during hyperosmotic condition. Under hyperosmotic condition, the vacuole remained enlarged in vac144 mutant, but this mutant phenotype is rescued when expressing wild-type Vac14 (Figure 3.7B). Expression of monomeric Vac14 point mutants in vac144 retained the enlarged vacuolar phenotype when exposed to hyperosmotic shock. Interestingly, in addition to the observed enlarged vacuole, the vacuolar membrane appears to form invaginations (Figure 3.7B). The vacuole in vac14 4 expressing Vac14 ${ }^{S S}$ and $V a c 14^{N G}$ remained enlarged similar to vac144 in both 
basal and in hyperosmotic conditions that stimulate hyperactive synthesis of $\operatorname{Ptd} \operatorname{Ins}(3,5) \mathrm{P}_{2}$, thus suggesting that the formation of Vac14 multimer is important for the synthesis of $\operatorname{PtdIns}(3,5) \mathrm{P}_{2}$.

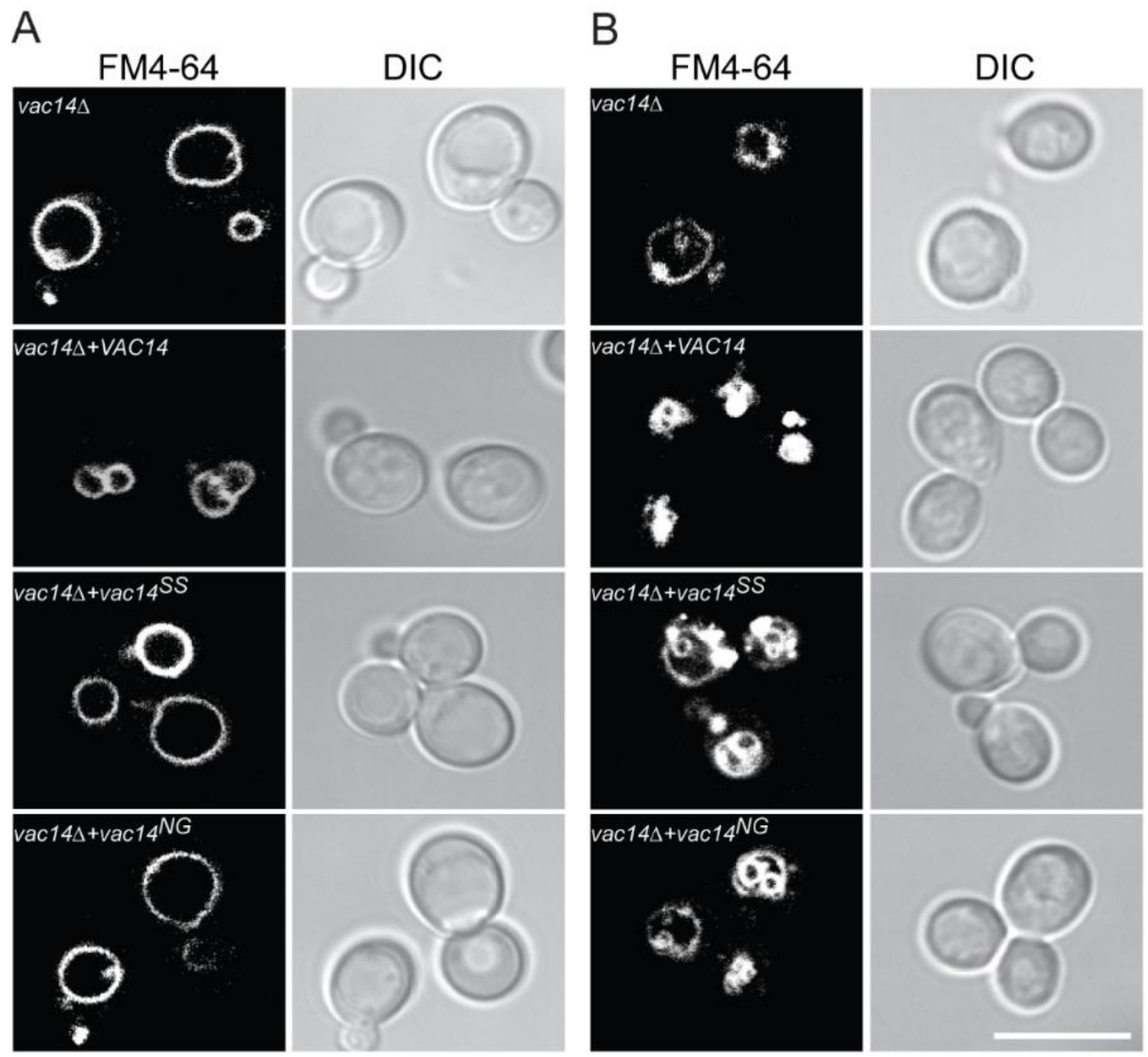

Figure 3. 7 Vacuolar morphology of vac144 expressing Vac14 point mutants.

Fluorescence images of yeast vacuoles labeled with FM4-64. vac144 mutants expressing wildtype Vac14 and vac14 point mutants, $v a c 14^{S S}$ and $v a c 14^{N G}$ in basal condition (A) and under $0.9 \mathrm{M}$ $\mathrm{NaCl}$ hyperosmotic condition (B). DIC: Differential interference contrast images of corresponding fluorescent images. 


\subsection{The role of PtdIns $(3,5) \mathrm{P}_{2}$ in vacuolar and lysosomal acidification}

\subsubsection{Loss of quinacrine accumulation in vacuoles of PtdIns $3,5 \mathrm{P}_{2}$-deficient yeast}

Quinacrine is an acidotropic dye often used as a qualitative assay to test for vacuolar

acidification. Upon entering the vacuole, quinacrine is protonated, which results in its fluorescence. Protonation of quinacrine also prevents it from exiting the vacuole. Therefore, quinacrine is mainly observed in the vacuole. It was shown previously that the vacuole of $f a b 1 \Delta$ yeast failed to accumulate quinacrine, which suggests that vacuolar acidification is defective (83, $89,108)$. To confirm that the $\operatorname{PtdIns}(3,5) \mathrm{P}_{2}$-deficient vacuole failed to accumulate quinacrine, cells were doubly labeled with quinacrine as well as CMAC (Figure 3.8). CMAC is a vacuolar probe that readily accumulates in the vacuole irrespective of vacuolar acidification. Using fluorescence microscopy, vacuoles of wild-type yeast accumulated both quinacrine and CMAC. As suggested by the literature, CMAC-labeled vacuoles in fabld mutant failed to acquire quinacrine similar to vph14 mutant, where quinacrine also failed to accumulate in CMAC-labeled vacuoles (Figure 3.8). Vph1p is a subunit of the vacuolar-ATPase $V_{0}$ domain found on the vacuolar membrane. Deletion of Vph1p results in the loss of a functional ATPase on the vacuole and therefore inability to properly acidify vacuoles. The failure to accumulate quinacrine in both $f a b 1 \Delta$ and $v p h 1 \Delta$ mutants, suggests that there is a similar functional defect of vacuolar acidification. However, quinacrine is only a qualitative measurement of vacuolar acidification. The minimal acidic level required for the sequestration of vacuolar quinacrine is unknown. Therefore, a method to quantitatively measure the vacuolar $\mathrm{pH}$ is required to observe the impact of $\operatorname{PtdIns}(3,5) \mathrm{P}_{2}$-deficiency on vacuolar acidification in fabld mutant. 


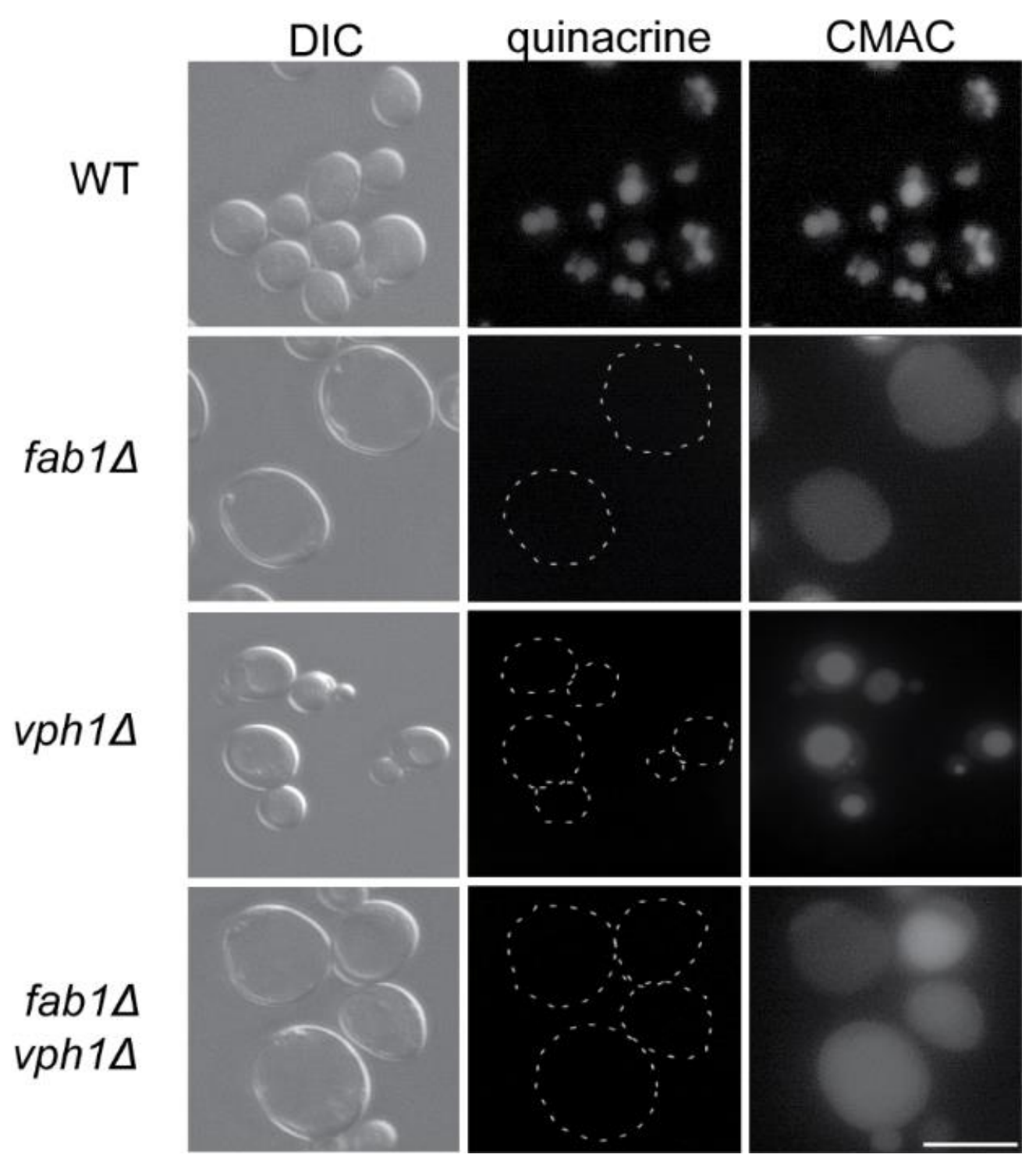

Figure 3. 8 Vacuolar accumulation of quinacrine.

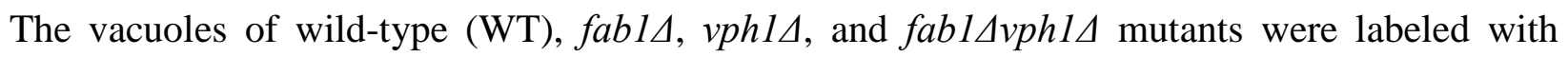
$100 \mu \mathrm{M}$ CMAC for $15 \mathrm{mins}$ in the dark, followed by $200 \mu \mathrm{M}$ quinacrine for $10 \mathrm{~min}$. Live-cell images were captured by fluorescence microscopy. Corresponding differential interference contrast (DIC) images are presented (scale bar $=10 \mu \mathrm{m})$. 


\subsubsection{Vacuolar accumulation of Mup1-pHluorin}

Fluorescence markers, such as quinacrine and CMAC, are used as general markers of the vacuole, and cannot be used to quantify $\mathrm{pH}$. Super-ecliptic pHluorin (pHluorin), a variant of GFP, is highly sensitive to $\mathrm{pH}$. The fluorescence intensity of pHluorin changes depending on the $\mathrm{pH}$ of the local environment, where the fluorescence intensity is quenched with lower $\mathrm{pH}$ (117). Therefore, $\mathrm{pHluorin}$ can be used as a quantitative tool for $\mathrm{pH}$ analysis. In order to evaluate the $\mathrm{pH}$ status of the vacuole, yeast containing the genomic Mup1-pHluorin was obtained from the Wendland lab. Mup1-pHluorin was first used as a tool for kinetic analysis of endocytosis in yeast (117). Mup1, is a methionine transporter that is located on the plasma membrane in the absence of methionine. While in the presence of methionine, Mup1 is internalized and transported to the vacuole via the MVB pathway (Figure 3.9A) (118). The pHluorin is fused to the cytoplasmic tail of Mup1, therefore, when Mup1-pHluorin is located on the plasma membrane, pHluorin is exposed to the neutral environment of the cytosol. With the addition of methionine, as Mup1-pHluorin is internalized, pHluorin is exposed to a more acidic environment as it enters the MVB and eventually the vacuolar lumen. Due to the $\mathrm{pH}$ sensitivity of pHluorin, the fluorescence intensity decreases as pHluorin changes its cellular location from the cytosol to the vacuole (Figure 3.9B) (117). Although pHluorin is different from GFP, as it is highly sensitivity to $\mathrm{pH}$, it remains resistant to proteolysis in the vacuole as the traditional GFP. These two important attributes of pHluorin are key for $\mathrm{pH}$ analysis of the yeast vacuole.

To prepare for $\mathrm{pH}$ measurement, cells containing Mup1-pHluorin grown to mid-log phase are exposed to $20 \mu \mathrm{g} / \mathrm{ml}$ methionine for 2 hours to allow vacuolar localization of Mup1-pHluorin. Fluorescence intensity of vacuolar pHluorin was recorded as basal fluorescence when cells were re-suspended in media buffered to $\mathrm{pH}$ 7.5. CCCP (carbonyl cyanide m-chlorophenyl hydrazone) is an ionophore that readily intercalates into the membrane bilayer and facilitates the free 
movement of protons $(119,120)$. Addition of CCCP mediates the movement of protons down their gradient into the cytosol and medium. As a result, the $\mathrm{pH}$ of the vacuole is forced to equilibrate to the media $\mathrm{pH}$ of 7.5 . The fluorescence intensity of pHluorin after equilibration to $\mathrm{pH} 7.5$ was recorded. The difference in fluorescence intensity before and after equilibration is used for calculating the relative basal vacuolar $\mathrm{pH}$.
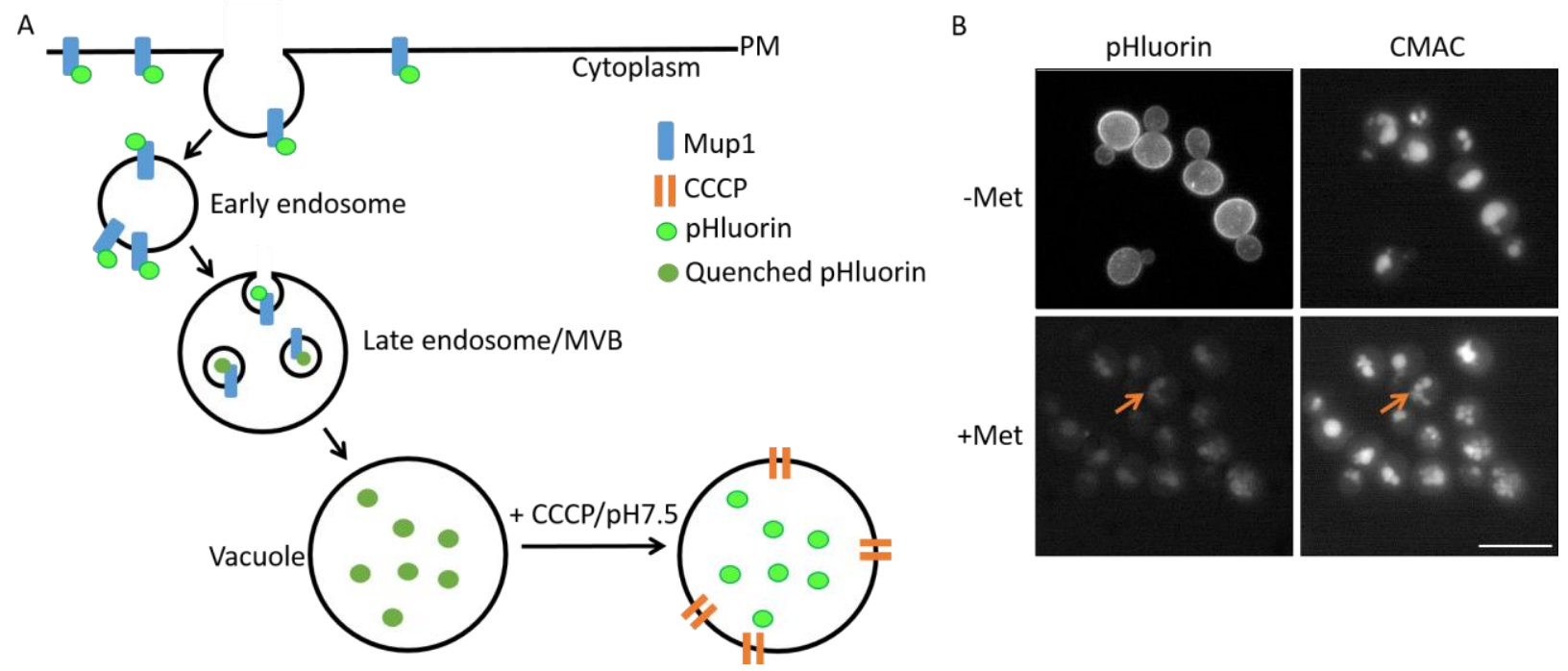

Figure 3. 9 The transport of Mup1-pHluorin to the vacuole through the endocytic pathway.

(A) The presence of excess methionine triggers the internalization of Mup1-pHluorin into endocytic vesicles. As the ESCRT machinery package receptors into internal vesicles to form multivesciular body (MVB), pHluorin which is fused to the cytoplasmic tail of Mup1 is trapped inside the MVB. As the local environment becomes increasingly acidic as MVB fuses with the vacuole, the fluorescence of pHluorin is quenched. However, pHluorin is unquenched by equilibrating the vacuolar $\mathrm{pH}$ to 7.5 with the addition of CCCP and incubation in media buffered to $\mathrm{pH}$ 7.5. (B) Example of wild-type cells expressing Mup1-pHluorin were labeled with $100 \mu \mathrm{M}$ CMAC and imaged with fluorescence microscopy before and after addition of methionine. CMAC-labeled vacuoles with corresponding pHluorin containing vacuoles (arrow). (Scale bar = $10 \mu \mathrm{m})$. 
To observe the vacuolar $\mathrm{pH}$ status of $f a b 1 \Delta$ and $v p h 1 \Delta$, fabl $1 \Delta$ Mup1-pHluorin and $v p h 1 \Delta$ Mup1-pHluorin were first generated by the yeast mating method. The fluorescence signal of pHluorin in CMAC-labeled vacuoles was weak in wild-type, fabld, and $v p h 1 \Delta$ (Figure 3.10A, b, $\mathrm{e}$, and $\mathrm{h}$, respectively). The weak signal observed was a result of quenching of $\mathrm{pHluorin}$ by the $\mathrm{pH}$ environment of the vacuole. By forcing the vacuolar $\mathrm{pH}$ to equilibrate to $\mathrm{pH} 7.5$ with $\mathrm{CCCP}$, the fluorescence signal of vacuolar pHluorin increased in wild-type, fabld and vphlA (Figure 3.10A $\mathrm{c}, \mathrm{f}$, and $\mathrm{i}$, respectively). The extent of quenching of pHluorin is dependent on the acidity of the vacuole, therefore, the ratio in fluorescence signal after equilibration to $\mathrm{pH} 7.5$ can be used to elucidate the $\mathrm{pH}$ status of the vacuole during basal condition. Shockingly, the fold-increase in fluorescence signal in wild-type and $f a b 1 \Delta$ was not significantly different. Wild-type and $f a b 1 \Delta$ both exhibited a similar increased in fluorescence intensity of $6.1 \pm 1.9$ and $5.5 \pm 0.67$, respectively (Figure 3.10B). In contrast, vphl 14 had only a $3.4 \pm 0.63$-fold increase in fluorescence intensity (Figure 3.10B). The reduced fold-increase in fluorescence intensity observed in $v p h 1 \Delta$ is expected due to the loss of vacuolar acidification by V-ATPase, resulting in a more neutral vacuole. However, the similarity in fluorescence intensity between wild-type and fabl $\Delta$ suggests that the basal vacuolar $\mathrm{pH}$ status of $f a b 1 \Delta$ is similar to wild-type. This contradicts the result from the quinacrine assay, where quinacrine failed to accumulate in the fabl $\Delta$ mutant. Failure of quinacrine to accumulate in the vacuolar lumen has long been recognized as an indication for an alkalinized vacuole. In addition, $\operatorname{PtdIns}(3,5) \mathrm{P}_{2}$ was also shown to directly interact with $\mathrm{Vph} 1$ and potentially acts to stabilize V-ATPase assembly and function under both hyperosmotic and glucose deprivation conditions (94). 


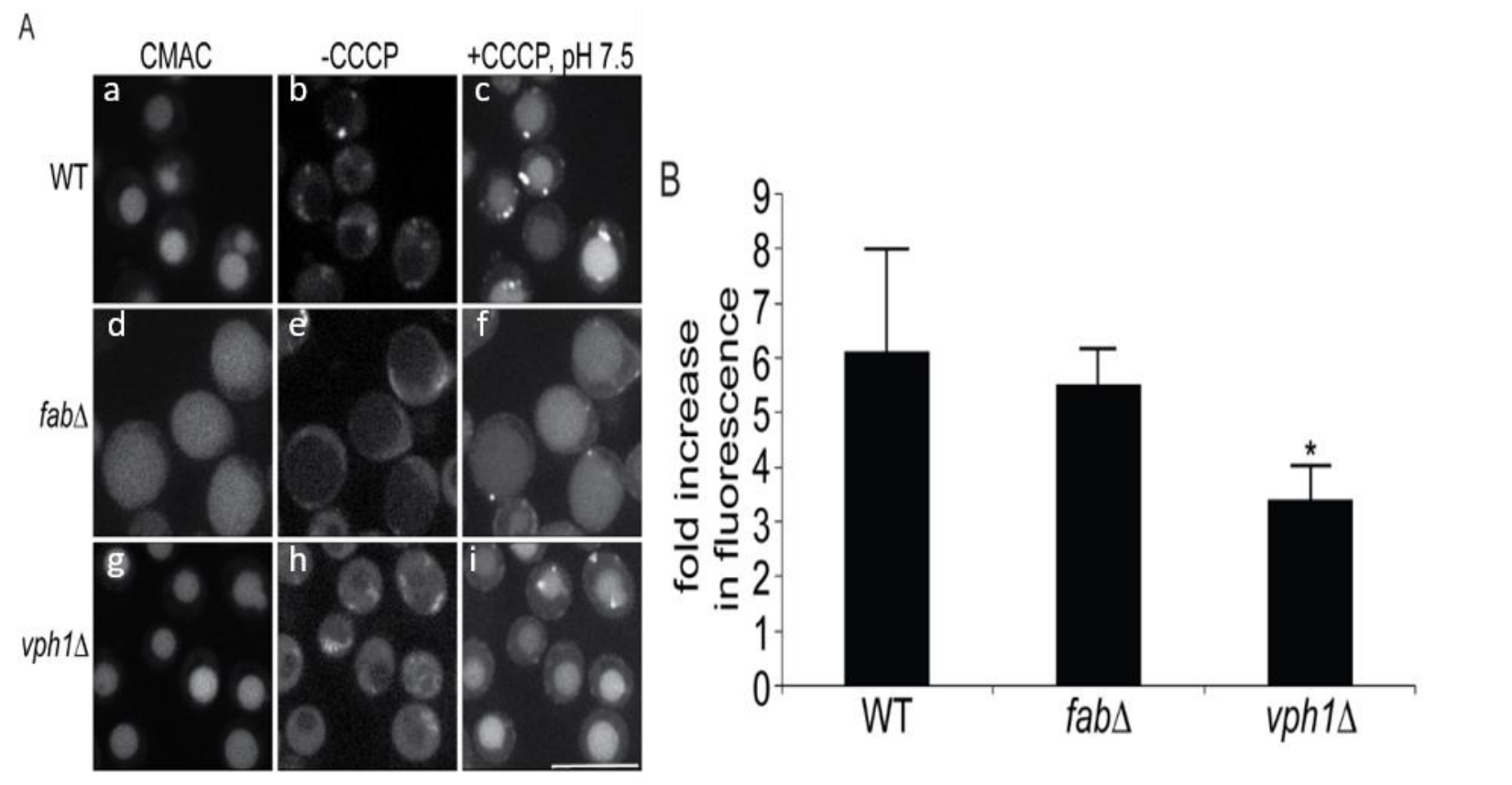

Figure 3. 10 The use of pHluorin for examining vacuolar $\mathrm{pH}$ status.

(A) Wild-type Mup1-pHluorin, fab1 Mup1-pHluorin, and vph1 Mup1-pHluorin were labeled with $100 \mu \mathrm{M}$ CMAC (a, d, g, respectively) in the presence of methionine. Images were captured before (b, e, and h, respectively) and after the addition of CCCP (c, f, and i, respectively) in media with pH7.5 using fluorescence microscopy. (Scale bar $=10 \mu \mathrm{m})$. (B) Measured fold-increase in fluorescence of pHluorin in wild-type Mup1-pHluorin ( $\mathrm{n}=514$ vacuoles), fab1 $\Delta$ Mup1-pHluorin ( $\mathrm{n}=372$ vacuoles), and vph14 Mup1-pHluorin $(\mathrm{n}=324$ vacuoles) after equilibration of vacuolar $\mathrm{pH}$ to 7.5 with the addition of CCCP. 


\subsubsection{Quantification of vacuolar $\mathrm{pH}$}

The results from the Mup1-pHluorin assay is inconsistent with the quinacrine assay, which suggests that $f a b l \Delta$ has a more alkaline $\mathrm{pH}$. Therefore, an additional quantitative assay was used to measure vacuolar $\mathrm{pH}$ to decipher the effect of loss of $\operatorname{PtdIns}(3,5) \mathrm{P}_{2}$ in $f a b 1 \Delta$ on vacuolar $\mathrm{pH}$. cDCFDA is a pH-sensitive fluorescent dye that accumulates in the vacuole of yeast. Although the fluorescence intensity of cDCFDA is dependent on vacuolar $\mathrm{pH}$, vacuolar cDCFDA accumulation is irrespective of vacuolar $\mathrm{pH}$ (121). As demonstrated, CMAC-labeled vacuoles of wild-type, fab1A, and $v p h 1 \Delta$ yeast accumulated cDCFDA (Figure 3.11). To quantify vacuolar $\mathrm{pH}$, fluorimetry was used to measure the fluorescence intensity of cells labeled with cDCFDA. First, labeled cells were separated into equal aliquots for measuring fluorescence intensity. An aliquot was used for measuring basal fluorescence intensity, while remaining aliquots were treated with CCCP for 4 min to equilibrate vacuolar $\mathrm{pH}$ of known media $\mathrm{pH}$ (Figure 3.12). The fluorescence intensity of the vacuolar $\mathrm{pH}$ equilibrated to the various media $\mathrm{pH}$ was used to generate an internal calibration

curve (Figure 3.13A). The calibration curve was then used for calculating the basal vacuolar $\mathrm{pH}$ based on recorded basal fluorescence intensity (Figure 3.13B). 


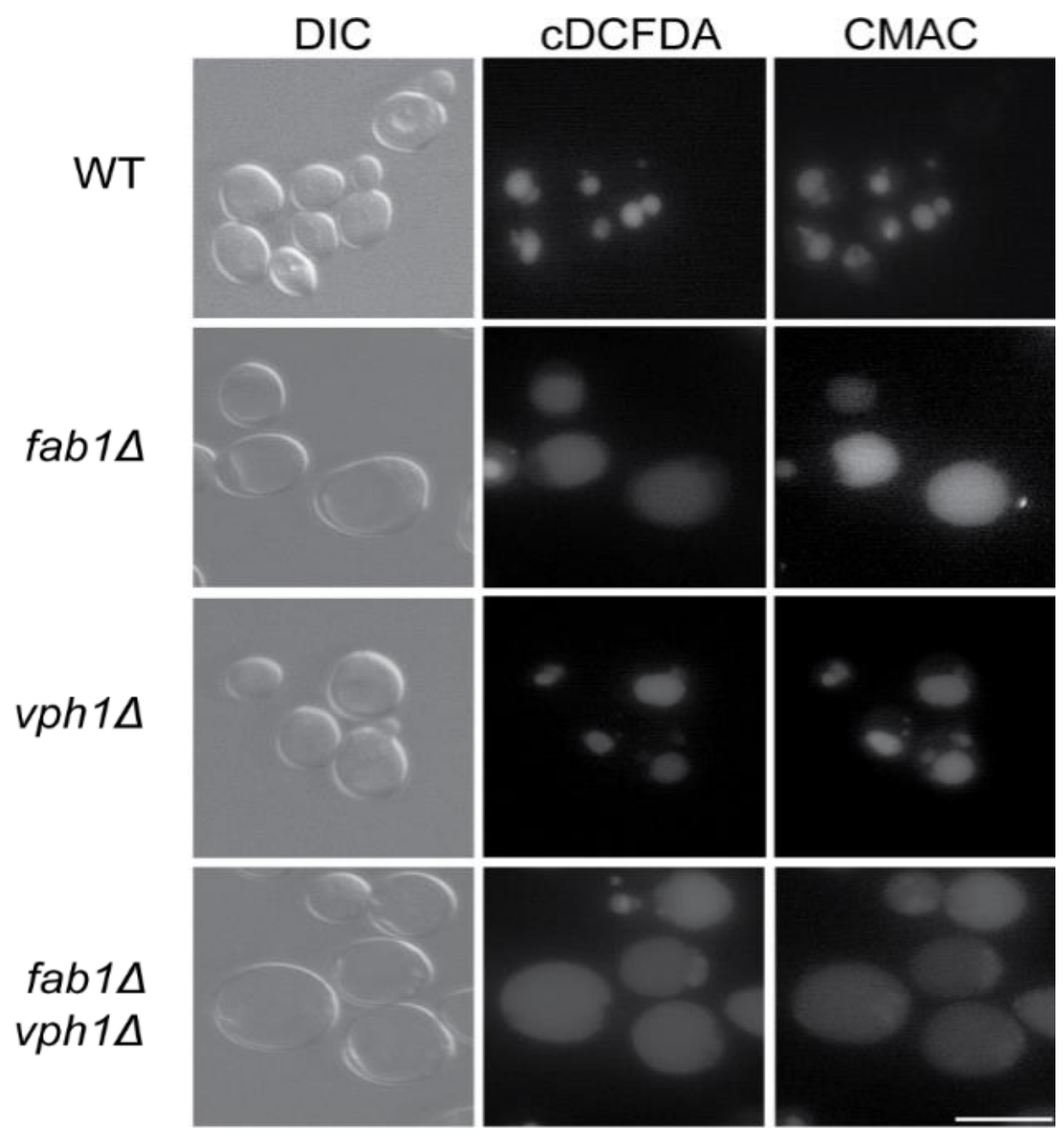

Figure 3. 11 Vacuolar localization of cDCFDA.

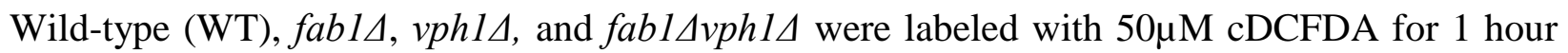
followed by $100 \mu \mathrm{M}$ CMAC for $15 \mathrm{~min}$ in $\mathrm{SC}$ media buffered to $\mathrm{pH}$ 7.5. Images were captured using fluorescence microscopy, corresponding differential interference contrast (DIC) images are presented. $($ Scale bar $=10 \mu \mathrm{m})$. 


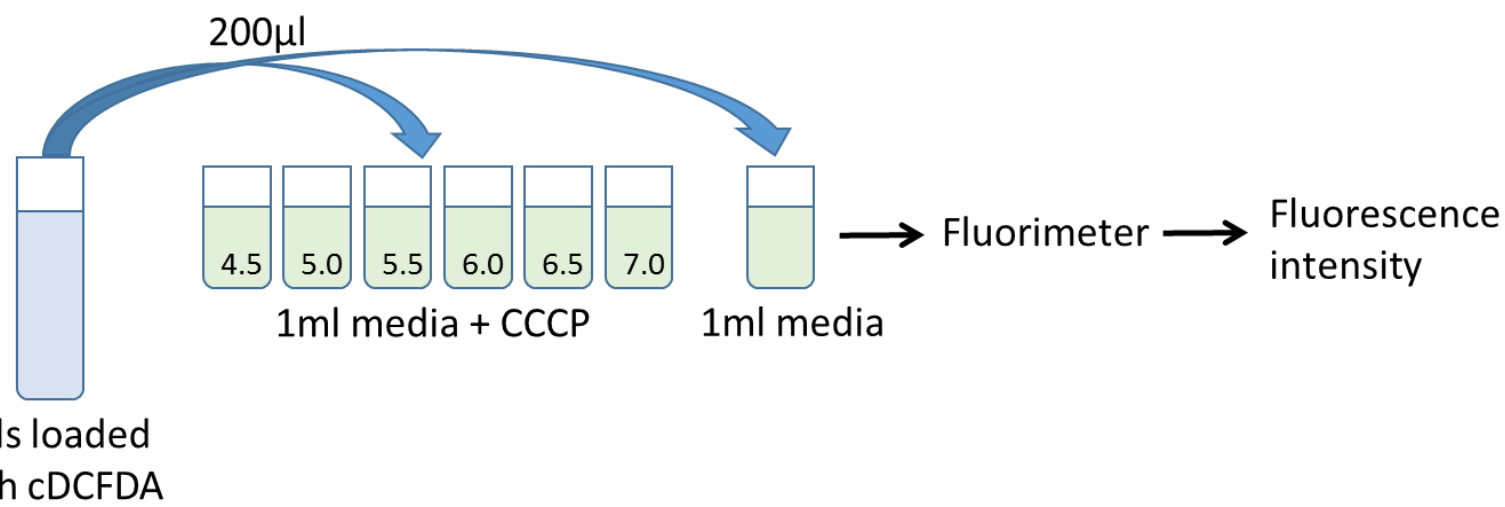

Figure 3. 12 Diagram illustrating the process of measuring fluorescence intensity of cDCFDA using fluorimetry.

Cells labeled with $50 \mu \mathrm{M}$ cDCFDA were transferred to individual $1 \mathrm{ml}$ aliquoted medium or medium buffered to indicated $\mathrm{pH}$ supplemented with $50 \mu \mathrm{M}$ CCCP. Cells were incubated in the respective media for $4 \mathrm{~min}$ and immediately transferred to the fluorimeter. Fluorescence intensity was measured using excitation scan between $400-520 \mathrm{~nm}$ and emission fluorescence intensity at $535 \mathrm{~nm}$. 

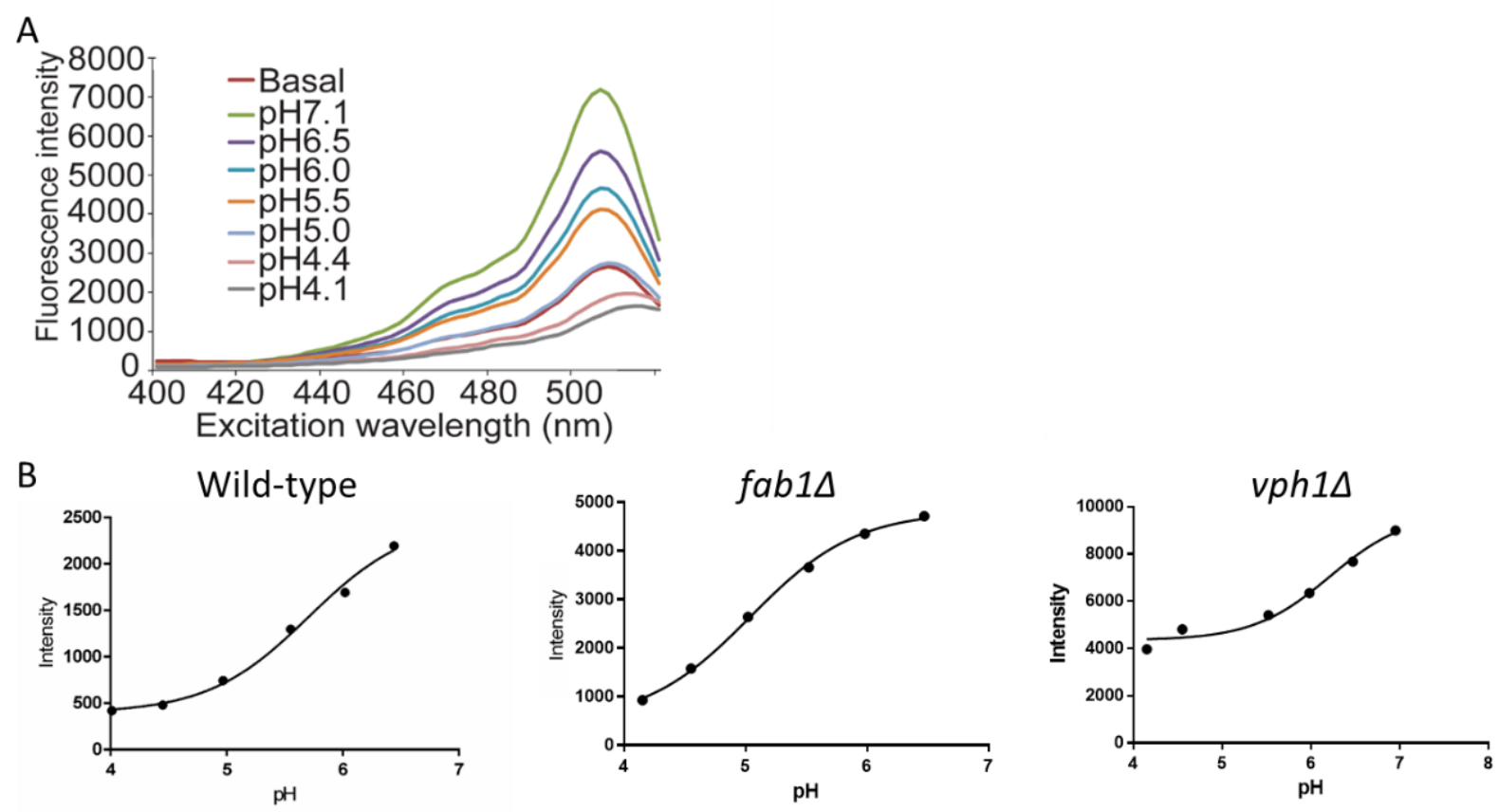

Figure 3. 13 Example of fluorescence intensity and calibration curves for quantification of vacuolar $\mathrm{pH}$.

(A) Fluorescence emission spectra of wild-type labeled with cDCFDA in buffer solutions of different pH. (B) Sigmoidal-fitted calibration curves of fluorescence intensity for cDCFDA of wild-type, $f a b 1 \Delta$, and $v p h 1 \Delta$ at $510 \mathrm{~nm}$ as a function of $\mathrm{pH}$. 
As expected, loss of $\mathrm{Vph} 1 \mathrm{p}(v p h 14)$ resulted in a more neutral vacuolar $\mathrm{pH}$ of $6.1 \pm 0.2$ (Figure 3.14A). Interestingly, the measured vacuolar $\mathrm{pH}$ of $f a b 1 \Delta$ was $4.9 \pm 0.1$, and is comparable to wild-type vacuolar $\mathrm{pH}$ of $4.9 \pm 0.2$ (Figure 3.14A). The vacuole of $f a b 1 \Delta$ yeast appears to remain acidic which contradicts the results from the quinacrine assay. In addition, a different background strain of yeast, BY4741, was used to show that the vacuolar $\mathrm{pH}$ observations were not strain specific. Both wild-type and fab1A in the BY4741 background showed similar vacuolar pH of

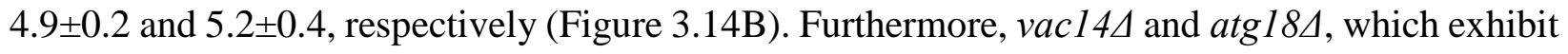
of $\sim 10 \%$ and 5-to-10 fold of wild-type PtdIns(3,5) $\mathrm{P}_{2}$, respectively, were used to test the effect of different levels of PtdIns(3,5) $\mathrm{P}_{2}$ on vacuolar acidification. The vacuolar $\mathrm{pH}$ of vac14 4 and $\operatorname{atg} 18 \Delta$ were measured to be $4.9 \pm 0.2$ and $4.8 \pm 0.1$, respectively. (Figure 3.14A). There were no significant differences between these strains, wild-type and fabld, which suggests that different levels of PtdIns(3,5) $\mathrm{P}_{2}$ do not affect basal vacuolar $\mathrm{pH}$.

During osmotic shock, PtdIns(3,5) $\mathrm{P}_{2}$ interacts with Vph1, to regulate V-ATPase activity during hyperosmotic and glucose-deprivation conditions (94). To investigate if the acidic vacuole observed in $f a b 1 \Delta$ was due to the presence of a functional V-ATPase, fabl $\Delta v p h 1 \Delta$ double mutant was generated by yeast mating. The loss of functional V-ATPase in fabl $\Delta v p h 1 \Delta$ resulted in alkalinization of the vacuole to $\mathrm{pH}$ of $6.1 \pm 0.2$, comparable to vph1 (Figure 3.14A). Wild-type and fabld were treated with concanacmycin A (conA), a potent V-ATPase inhibitor, to confirm that the observed acidic vacuolar $\mathrm{pH}$ in fabld was due to the activity of V-ATPase. cDCFDAlabeled cells were treated with conA for an hour before measurements were recorded. The observed vacuolar $\mathrm{pH}$ was again comparable between wild-type and fabl $\Delta(\mathrm{pH} 5.8 \pm 0.1$ and $\mathrm{pH}$ $5.9 \pm 0.2$, respectively) after ConA treatment (Figure 3.15). The alkaline vacuolar $\mathrm{pH}$ observed in 


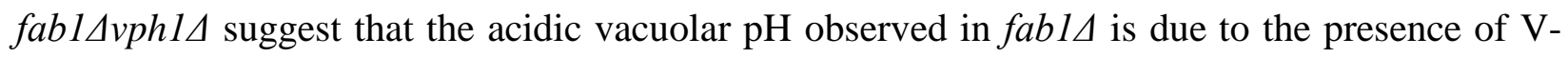
ATPase activity.

The measured vacuolar $\mathrm{pH}$ was based on steady state $\operatorname{PtdIns}(3,5) \mathrm{P}_{2}$ levels (Figure 3.14). During hyperosmotic shock, the level of PtdIns $(3,5) \mathrm{P}_{2}$ is known to increase $\sim 20$-fold within $10 \mathrm{~min}$ (81). To test the effect of hyperosmotic shock on vacuolar $\mathrm{pH}$, cells were first labeled with cDCFDA and measurements were recorded for every 5 mins after treatment with $0.9 \mathrm{M} \mathrm{NaCl}$. There was no significant difference between wild-type and fabld that were not exposed to salt shock, the vacuolar $\mathrm{pH}$ for both wild-type and fabl $\Delta$ remained approximately $\sim \mathrm{pH} 4.8$ for the entire duration (Figure 3.16). There was a slight alkalinization of vacuolar $\mathrm{pH}$ after salt shock in wildtype cells, but this was only significant $10 \mathrm{~min}$ post-treatment. Interestingly, the rate of alkalinization in vacuolar $\mathrm{pH}$ in fabld was significant immediately after hyperosmotic shock (Figure 3.16). The observed alkalinization of vacuolar $\mathrm{pH}$ in wild-type yeast suggests that hyperosmotic shock affects vacuolar $\mathrm{pH}$. In addition, the earlier alkalinization of vacuolar $\mathrm{pH}$ in

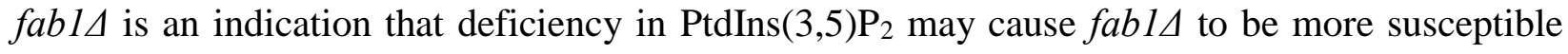
stress conditions. 


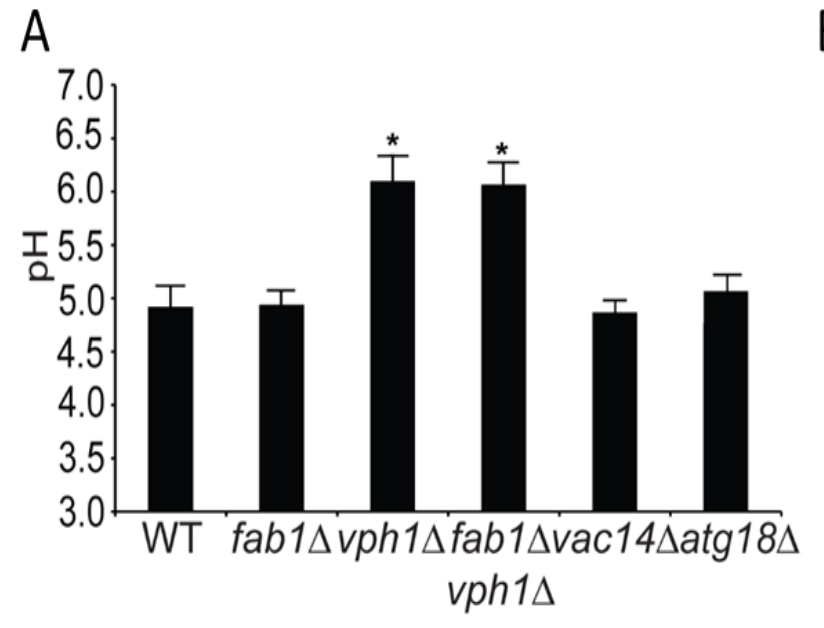

B

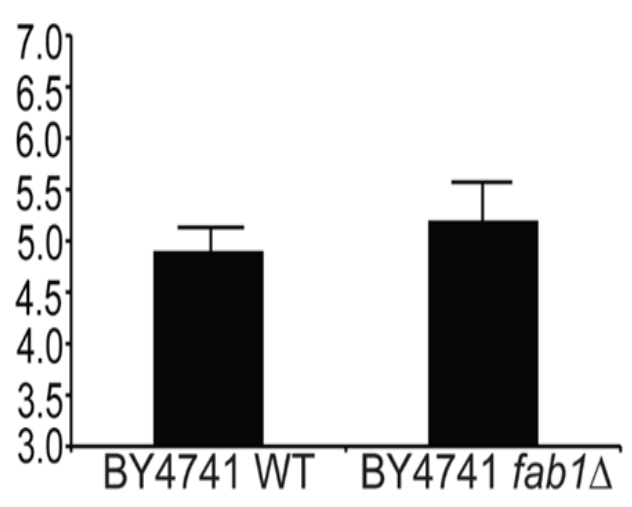

Figure 3. 14 Quantification of vacuolar $\mathrm{pH}$ in cells with different levels of $\operatorname{Ptd} \operatorname{Ins}(3,5) \mathrm{P}_{2}$.

(A) The calculated mean vacuolar $\mathrm{pH}$ for corresponding yeast strains (SEY6210 background) using respective internal calibration curves base on $n=$ number of independent experments. Wildtype $(\mathrm{n}=10)$, fabl $\Delta(\mathrm{n}=10), \operatorname{vph} 1 \Delta(\mathrm{n}=3)$, fabl $\Delta \operatorname{vph} 1 \Delta(\mathrm{n}=3), \operatorname{vac} 14 \Delta(\mathrm{n}=3)$, and $\operatorname{atg} 18 \Delta(\mathrm{n}$ =3). (B) The calculated mean vacuolar $\mathrm{pH}$ for wild-type $(\mathrm{n}=4)$ and fabld $(\mathrm{n}=3)$ in the BY4741 background strain. (*) significantly different based on two-way ANOVA and Turkey's post-hoc test against wild-type, $f a b 1 \Delta, v p h 1 \Delta, v a c 14 \Delta$, and $\operatorname{atg} 18 \Delta$. 


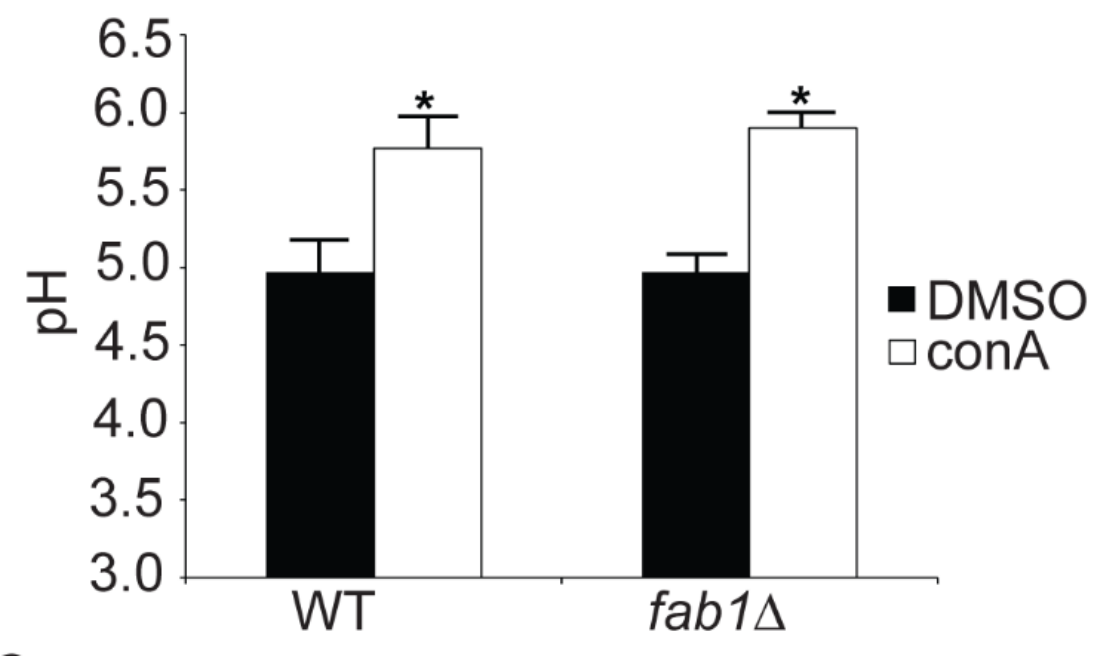

Figure 3. 15 Inhibition of V-ATPase activity neutralizes the vacuolar $\mathrm{pH}$.

Cells labeled with $50 \mu \mathrm{M}$ cDCFDA were treated with DMSO $(\mathrm{n}=3)$ or $1 \mu \mathrm{M}$ conA to inhibit VATPase activity $(\mathrm{n}=3)$. $\mathrm{pH}$ values were calculated based on fluorescence intensity measured by fluorimetry fitted into respective internal sigmoidal calibration curve. $\left.{ }^{*}\right)$ significantly different based on two-way ANOVA and Tukey’s post-hoc test against control (DMSO). 


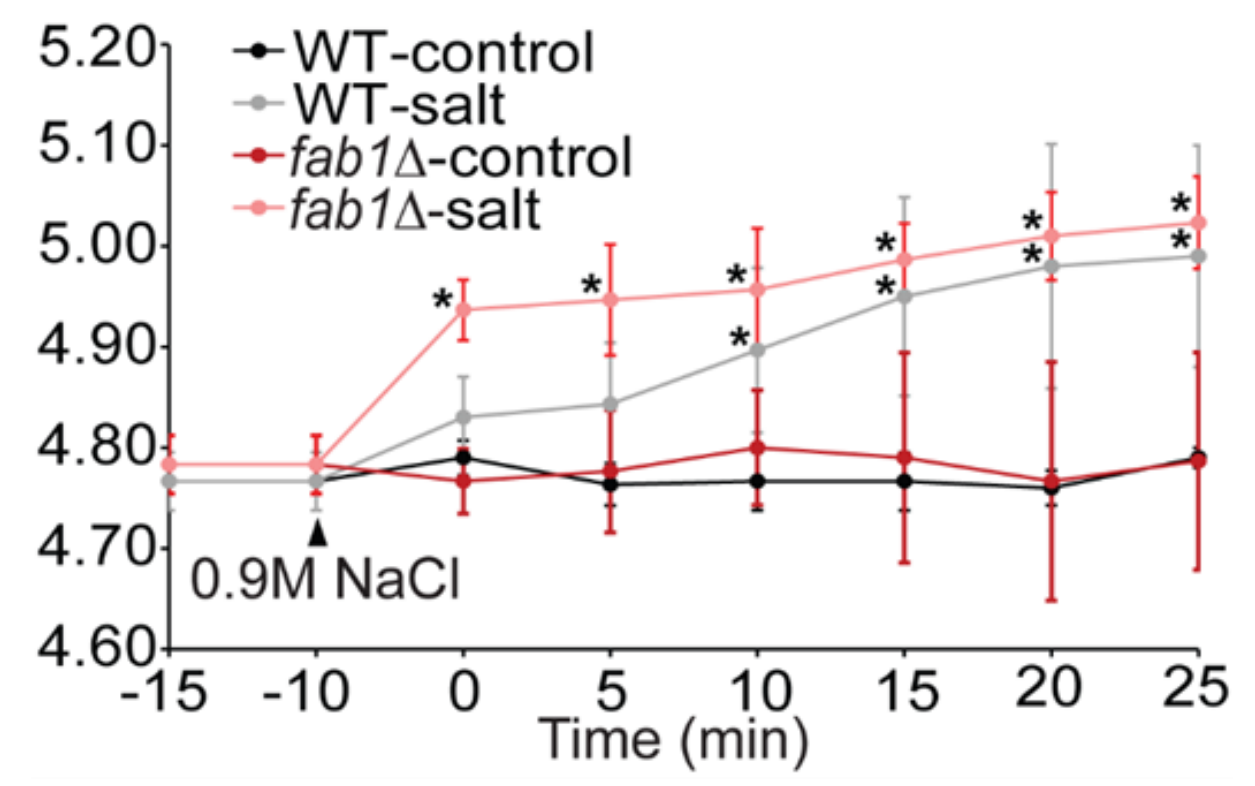

Figure 3. 16 Quantification of vacuolar $\mathrm{pH}$ of cells exposed to hyperosmotic shock.

Wild-type and fab1 1 labeled with $50 \mu \mathrm{M}$ cDCFDA were split into two equal pools, where one is used as control and another exposed to $0.9 \mathrm{M} \mathrm{NaCl}$. Fluorescence intensity was measured prior to addition of $0.9 \mathrm{M} \mathrm{NaCl}(-15$ and $-10 \mathrm{~min}), 10 \mathrm{~min}$ after exposure to $0.9 \mathrm{M} \mathrm{NaCl}(0 \mathrm{~min})$ and continuously measured at $5 \mathrm{~min}$ interval for $25 \mathrm{~min}$. (*) significantly different based on two-way ANOVA and Tukey's post-hoc test. 


\subsubsection{Quantification of lysosomal $\mathrm{pH}$}

RAW264.7 (RAW) macrophages were used to explore the role of $\operatorname{PtdIns}(3,5) \mathrm{P}_{2}$ in lysosomal acidification. To quantify lysosomal $\mathrm{pH}$, lysosomes were labeled with fluorescein isothiocyanate (FITC) - labeled dextran. FITC-dextran is readily taken up by cells via pinocytosis and accumulates in lysosomes (Figure 3.17). FITC is a pH-sensitive fluorescent dye that has dual fluorescence moieties, with one moiety that is $\mathrm{pH}$ dependent with an excitation at $485 \mathrm{~nm}$, while the second moiety is $\mathrm{pH}$-insensitive with an excitation $438 \mathrm{~nm}$. The ratio of $485 / 438 \mathrm{~nm}$ normalizes the differences in concentration of fluorophore, as well as photobleaching, and thereby represents fluorescence intensity that is based on lysosomal $\mathrm{pH}$. The fluorescence ratio was calibrated by equilibrating to extracellular $\mathrm{pH}$ using nigericin, a $\mathrm{K}^{+} / \mathrm{H}^{+}$ionophore, and monensin, a $\mathrm{Na}^{+} / \mathrm{H}^{+}$ ionophore. The fluorescence ratio was recorded continuously in a series of calibration buffers of different $\mathrm{pH}$ values (Figure 3.18A). The fluorescence ratio was higher at alkaline $\mathrm{pH}$ values, and decreases with lower $\mathrm{pH}$ values (Figure 3.18A). The fluorescence ratios recorded at the various calibration $\mathrm{pH}$ were fitted to a sigmoidal calibration curve for calculating basal lysosomal $\mathrm{pH}$. As expected, control cells treated with DMSO has a measured lysosomal $\mathrm{pH}$ of $4.8 \pm 0.2$ (Figure 3.18B). To quantify the lysosomal $\mathrm{pH}$ in cells deficient in $\operatorname{Ptd} \operatorname{Ins}(3,5) \mathrm{P}_{2}$, cells were treated with PIKfyve inhibitors, apilimod and MF4, to inhibit the synthesis of PtdIns(3,5) $\mathrm{P}_{2}$. Lysosomal $\mathrm{pH}$ of cells treated with either apilimod or MF4 for 1 hour were $4.9 \pm 0.4$ and $4.7 \pm 0.1$, respectively (Figure 3.18B). In addition, cells were also treated with apilimod for 4 hours to test for the effect of prolonged loss of PtdIns(3,5) $\mathrm{P}_{2}$ on lysosomal $\mathrm{pH}$. Even with the loss of PtdIns $(3,5) \mathrm{P}_{2}$ over an extended duration, the lysosomal $\mathrm{pH}$ was found to be $5.1 \pm 0.2$, which was not statistically different from control (DMSO) or apilimod- and MF4-treated for only an hour (Figure 3.18B). In contrast, inhibiting the V-ATPase with conA resulted in alkalinization of lysosomal $\mathrm{pH}$ to $7.1 \pm 0.2$ (Figure 3.18B). Furthermore, consistent with the yeast studies, inhibiting V-ATPase activity in cells 
deficient in PtdIns(3,5) $\mathrm{P}_{2}$ also resulted in an alkalinization of lysosomal $\mathrm{pH}$ to $7.3 \pm 0.2$ (Figure 3.18B). Therefore, both the yeast and mammalian studies indicate that $\operatorname{Ptd} \operatorname{Ins}(3,5) \mathrm{P}_{2}$ is not involved in the maintenance of steady-state vacuolar/lysosomal $\mathrm{pH}$.

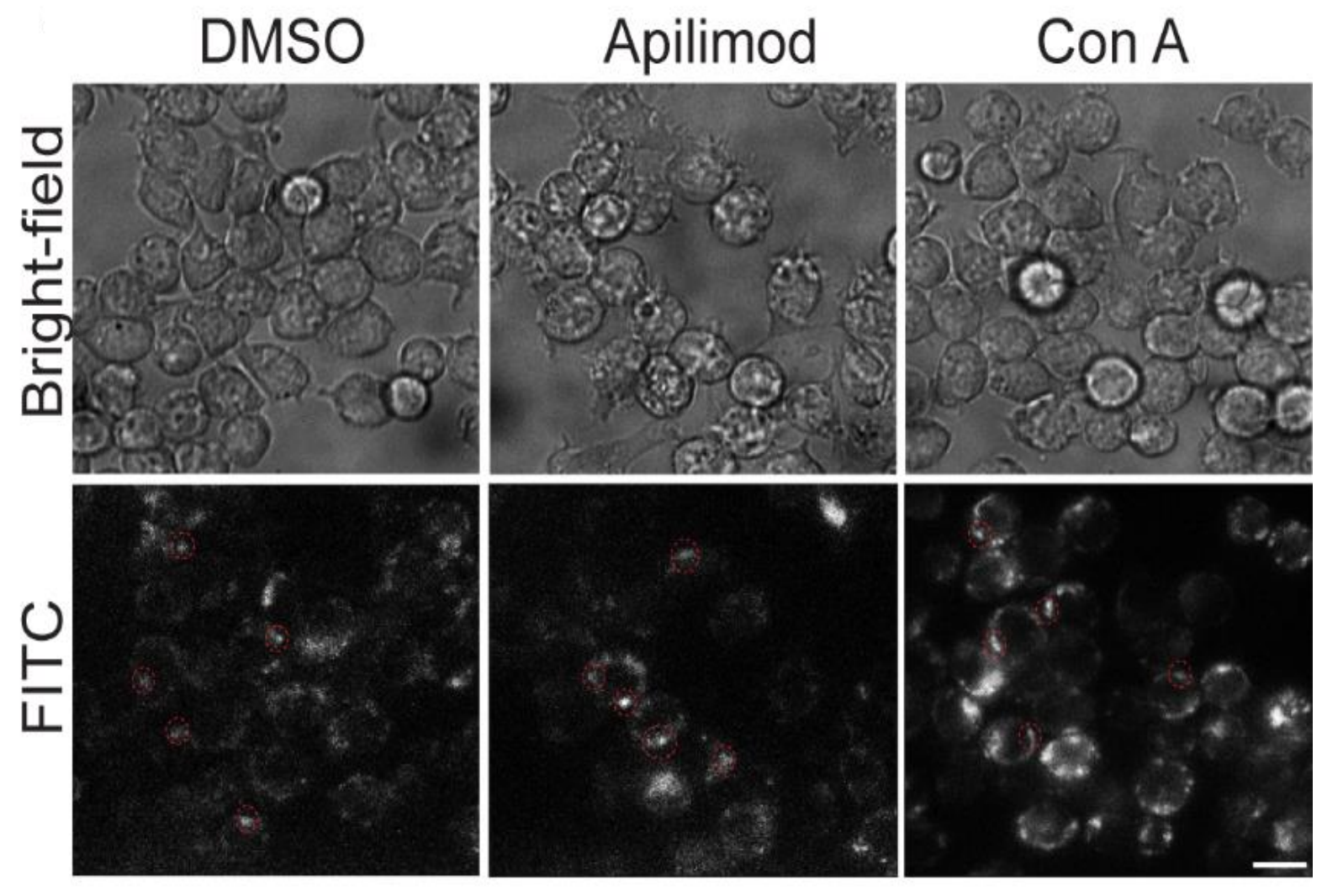

Figure 3. 17 Lysosomal accumulation of FITC-dextran.

RAW 264.7 macrophages were labeled with $2 \mathrm{mg} / \mathrm{ml}$ FITC-dextran for 1 hour. Internalised FITCdextran were chased for 1 hour before drug treatment with either $20 \mu \mathrm{M}$ apilimod, $1 \mu \mathrm{M}$ ConA, or equal of DMSO for 1 hour. Images were taken with fluorescence ratiometric microscopy and corresponding bright-field images are presented. (Circle) examples of random FITC-labeled lysosomes selected for measurements. (Scale $=5 \mu \mathrm{m})$. 

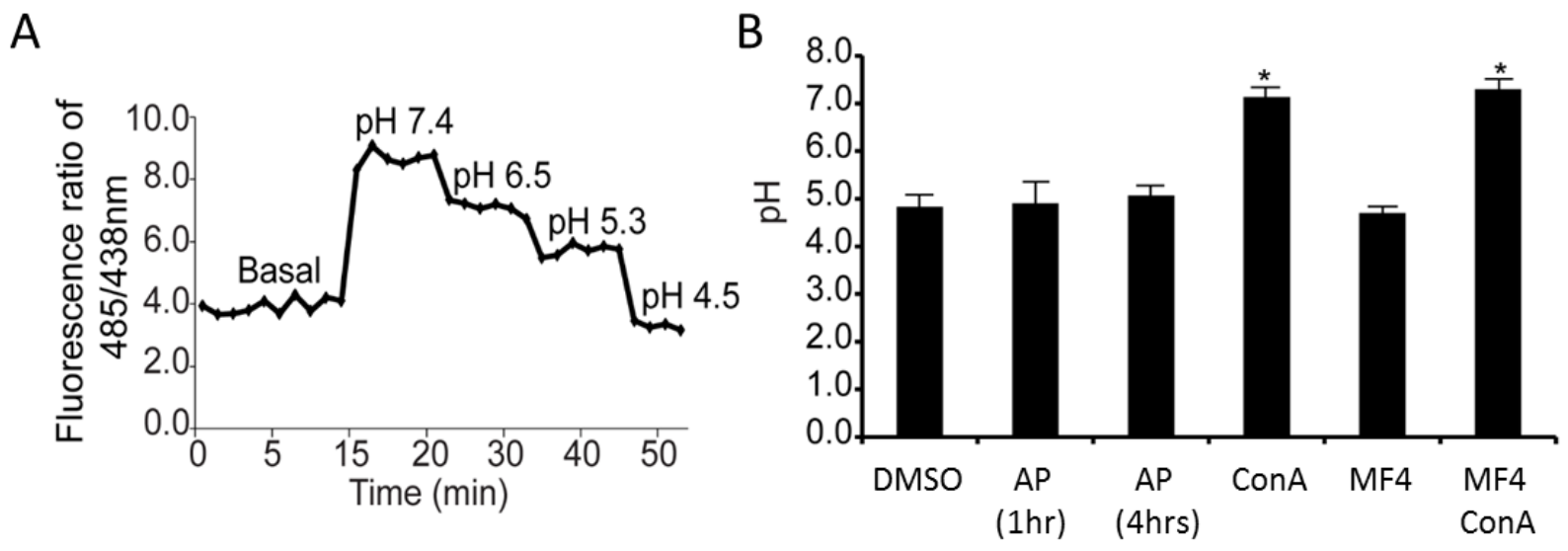

Figure 3. 18 Lysosomal acidification is not affected by PIKfyve inhibition.

Lysosomes in RAW 267.4 macrophages were labeled with FITC-dextran. Ratiometric imaging was used to measure the basal fluorescence intensity of lysosomal FITC-dextran. Cells were then treated with monensin and nigericin containing media with known $\mathrm{pH}$ ranging from 7.5 to 4.5 . Fluorescence intensity was measured at each interval to generate an internal calibration curve. (A) Example of an internal calibration curve used to convert fluorescence intensity ratio of FITC to lysosomal pH. (B) Lysosomal pH in RAW cells treated with DMSO ( $\mathrm{n}=362$ lysosomes), $20 \mathrm{nM}$ apilimod for $1 \mathrm{~h}$ ( $\mathrm{n}=412$ lysosomes), $20 \mathrm{nM}$ apilimod for $4 \mathrm{~h}$ ( $\mathrm{n}=125$ lysosomes), $200 \mathrm{nM}$ MF4 ( $\mathrm{n}=124$ lysosomes), or $1 \mu \mathrm{M}$ conA ( $\mathrm{n}=355$ lysosomes). (*) significantly different based on twoway ANOVA and Tukey's post-hoc test. 
Chapter 4: Discussion 


\subsection{Characterizing the importance of $\operatorname{PtdIns}(3,5) \mathrm{P}_{2}$}

$\operatorname{Ptd} \operatorname{Ins}(3,5) \mathrm{P}_{2}$ is a low abundance phosphoinositide that comprises only $\sim 0.1 \%$ of total cellular phosphoinositides (80-82). Due to its low abundance, the function and regulation of PtdIns $(3,5) \mathrm{P}_{2}$ has only recently started to unravel. Although the exact functional mechanism of PtdIns(3,5) $\mathrm{P}_{2}$ has yet to be deciphered, it has been linked to a number of cellular functions. One of the noticeable functions is the regulation of vacuolar/endolysosomal morphology. Cells deficient in PtdIns(3,5) $\mathrm{P}_{2}$ exhibit enlarged vacuolar/endolysosomal structures. The change in organelle morphology is speculated to be an indirect result of defective membrane trafficking (86, $88,89)$. In addition, $\operatorname{Ptd} \operatorname{Ins}(3,5) \mathrm{P}_{2}$ has also been suggested to be involved in the regulation of ion channel activities, such as vacuolar $\mathrm{H}^{+}$-ATPase and the mucolipin $\mathrm{Ca}^{2+}$ release channel (TRPML1) (91-93).

To fully understand the function of $\operatorname{Ptd} \operatorname{Ins}(3,5) \mathrm{P}_{2}$, it is important to map out the mechanism involved in the regulation of $\operatorname{Ptd} \operatorname{Ins}(3,5) \mathrm{P}_{2}$. $\operatorname{Ptd} \operatorname{Ins}(3,5) \mathrm{P}_{2}$ is predominantly synthesized on the vacuole/endolysosome by the PtdIns(3)P 5-kinase, Fab1/PIKfyve, while the antagonist phosphatase, Fig4/Sac3, converts PtdIns(3,5) $\mathrm{P}_{2}$ back to PtdIns(3)P (Figure 1.8). Interestingly, both the kinase and phosphatase exist in a single complex with Vac14/ArPIKfyve. Vac14 acts as an adaptor protein that links the two enzymes together to form the Fab1 complex. The Fab1 complex is necessary for the regulation of $\operatorname{Ptd} \operatorname{Ins}(3,5) \mathrm{P}_{2}$, although how the two enzymes exist in harmony is unknown. To further complicate the mechanism, there may be additional regulators involved, such as Vac7 and Atg18 (Figure 1.8) (83, 107).

The first step toward understanding the protein network in the regulation of $\operatorname{PtdIns}(3,5) \mathrm{P}_{2}$ is to decipher the importance and role of Vac14. Previous studies indicate that Vac14 can form a 
homomeric complex, but is this relevant for the formation of the Fab1 complex and the regulation of $\operatorname{Ptd} \operatorname{Ins}(3,5) \mathrm{P}_{2}$ ?

\subsubsection{Vac14 homomerization via the C-terminus}

Vac14 is conserved from yeast to mammals and consists of multiple tandem HEAT repeats, which are known to mediate of protein-protein interactions. Two-hybrid analysis and cross-linking purification studies suggest that Vac14 interacts with another copy of Vac14 to form a multimer. To understand the role of Vac14 homomerization, the region responsible for self-association was dissected by using Vac14 truncated mutants. Based on preliminary data from co-IP analysis by T. Alghamdi, co-IP analysis suggests that the C-terminus is required for Vac14 self-interaction in yeast (116). A Vac14 mutant containing only the N-terminus, Vac14 ${ }^{4600-880}$, lost the ability to coIP wild-type Vac14-HA (Figure 4.1). The N-terminal residues 1-600 failed to interact with wildtype Vac14 suggesting that Vac14 homomerization requires the C-terminus. 


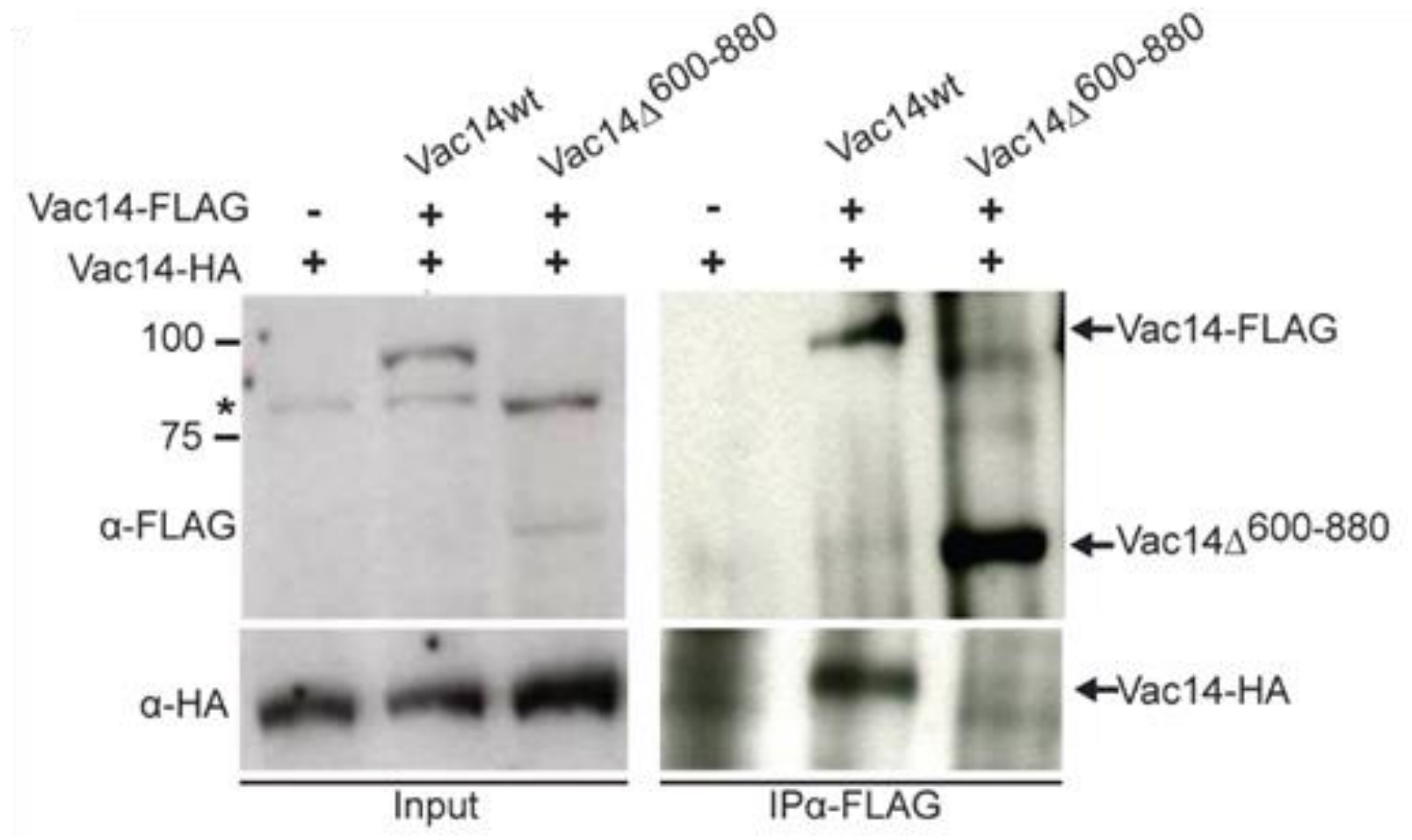

Figure 4. 1 The N-terminal region of Vac14 is not required for Vac14 homomerization.

Yeast expressing Vac14-HA was transformed with either empty plasmid (-), Vac14 ${ }^{\mathrm{WT}}$-FLAG or vac14 $4^{4600-800}$-FLAG. Immunoprecipitation was performed using monoclonal anti-FLAG antibodies. SDS-PAGE and Western blot were used to separate and probe for the presence of wildtype Vac14-HA and vac14 ${ }^{4600-800}$-FLAG using anti-HA and anti-FLAG antibodies, respectively (116). 
In this study, co-IP analysis confirmed that the Vac14 C-terminus (residues 557-880) alone was sufficient for interaction with wild-type Vac14 (Figure 3.1). In addition, in-vitro binding assay also supports that the Vac14 self-interaction domain resides in the C-terminus (Figure 3.2A). In contrast, the N-terminus alone (residues 1-334) was insufficient for binding to wild-type Vac14 (Figure 3.2A). The ability of recombinant Vac14 to interact in-vitro further confirms that the Vac14 C-terminus directly interacts with Vac14 to form the homomeric complex. This is consistent with the mammalian study that showed ArPIKfyve also interact with itself via the Cterminal 523-782 residues (113).

To further identify the Vac14 motif in the C-terminal region, point mutations in the most conserved motifs in the C-terminus were generated (Figure 3.2) (116). In the master thesis by T.Alghamdi, each mutant displays a degree of loss of Vac14 homomerization, with Vac14 ${ }^{S S}$ and Vac14 ${ }^{C R Y}$ showing the most significant ( 90\%) loss of interaction (116). Recombinant Vac14 point mutants $\left(\operatorname{Vac} 14^{\mathrm{SS}}, \mathrm{Vac} 14^{\mathrm{CRY}}, \mathrm{Vac} 14^{\mathrm{NG}}\right.$, and $\left.\mathrm{Vac} 14^{\mathrm{SIA}}\right)$ were used to test for in-vitro interaction (Figure 3.2B). All four mutants lost the ability to interact with wild-type Vac14 in the in-vitro affinity binding assay (Figure 3.2B). It was previously predicted that there are approximately 21 and 17 HEAT repeats spanning Vac14 and ArPIKfyve, respectively (109). As previously mentioned, HEAT repeats are known for scaffolding of protein-protein interactions and are often found in proteins that facilitate multi-protein complex formation, such as the protein phosphatase $2 \mathrm{~A}(110,122)$. Therefore, the identified point mutation region in this study may disrupt a potential HEAT repeat in the C-terminus (ie. residues 560 and 564 of $\mathrm{Vacl}^{\mathrm{SS}}$ ) that is responsible for Vac14 self-interaction. 


\subsubsection{Vac14 multimerization}

ArPIKfyve was previously predicted to form homodimer and/or homotrimer based on invivo cross-linking of protein complexes. IP analysis of purified ArPIKfyve cross-linked complexes revealed molecular weight of approximate 80-, 170-, and 250-kDa by immunoblotting (113). Considering the monomer ArPIKfyve has a molecular weight of $82 \mathrm{kDa}$, it was proposed that the purified ArPIKfyve consisted of a mixture of monomers, homodimers, as well as homotrimers. Although Sbrissa et al. stated that the captured ArPIKfyve homomeric complexes were not associated with PIKfyve and Sac3, this does not eliminate the possibility of the presence of other potential binding partners. In this study, recombinant Vac14 was used to characterize the homomeric state of yeast Vac14. Use of recombinant Vac14 allows the determination of its homomeric state and eliminate possible protein interactions.

To determine the multimeric state of Vac14, fast protein liquid chromatography (FPLC) and differential velocity ultracentrifugation with glycerol gradient were used to determine the molecular weights of recombinant T7-Vac14-His 6 and T7-Vac14 ${ }^{\mathrm{SS}}$-His 6 . Based on in-vitro binding assays, we confirmed that the purified recombinant wild-type T7-Vac14-His6 retains the ability to self-interact to form a homomeric complex. In addition, we have also shown that in both yeast and recombinant system, Vac14 $4^{\mathrm{SS}}$, cannot self-interact to form a homomeric complex, and therefore only exists as a monomeric protein (Figure 3.2B). FPLC and differential velocity ultracentrifugation separates proteins based on molecular mass and shape. By identifying the molecular mass difference between the monomeric Vac14 (T7-Vac14 ${ }^{\mathrm{SS}}-\mathrm{His}_{6}$ ), and the Vac14 homomeric complex (T7-Vac14 $\left.{ }^{\mathrm{wt}}-\mathrm{His}_{6}\right)$, we can then deduce the multimeric state of Vac14.

FPLC protein fractionation is based on the probability of globular proteins to enter and exit the porous matrix (123). The porous matrix is designed to allow proteins within a specific range of molecular mass to be separated effectively. Proteins with molecular mass outside of the 
fractionation range will not be fractionated (123). The result for FPLC separation of purified recombinant T7-Vac14 ${ }^{\mathrm{wt}}$-His 6 homomeric complex was inconclusive. Recombinant T7-Vac $14^{\mathrm{wt}}{ }_{\text {- }}$ His6 was detected in the void volume which indicates that the homomeric complex has a molecular weight greater than the upper fractionation range of $1,500 \mathrm{kDa}$ for the Sephacryl S-300 column (Figure 3.4A). The possibility of the recombinant T7-Vac14 ${ }^{\mathrm{wt}}-\mathrm{His}_{6}$ found in the void volume to be an insoluble aggregate was ruled out due to the ability of the same recombinant T7-Vac14 ${ }^{\mathrm{wt}}$-His 6 to retain the ability to interact with recombinant Vac14-S•tag (Figure 3.2B). Therefore, the presence of recombinant $\mathrm{T} 7-\mathrm{Vac} 14^{\mathrm{wt}}{ }_{-\mathrm{His}} 6$ in the void volume suggests that the Vac14 homomeric complex may consists of more than 10 copies of the monomer Vac14, given the monomer Vac14 has a molecular weight of $100 \mathrm{kDa}$. However, a more plausible explanation could be that the fractionation of the recombinant $\mathrm{T} 7-\mathrm{Vac}_{1} 4^{\mathrm{wt}}{ }_{-\mathrm{His}} 6$ is affected by the shape of the protein complex. As mentioned previously, the effective FPLC fractionation range is based on globular proteins (123). Although the structure of Vac14 is unknown, proteins that also contain multiple HEAT repeats, such as the protein phosphatase $2 \mathrm{~A}$, are known to form a non-globular, superhelical structure (122). Therefore, if recombinant T7-Vac14 ${ }^{\mathrm{wt}}-\mathrm{His}_{6}$ has a large Stoke's radius (nonglobular), this may restrict its movement through the porous matrix and hence its migration pattern will not reflect its actual molecular weight. This was supported by the separation of the monomeric $\mathrm{T} 7-\mathrm{Vac} 14^{\mathrm{SS}}$-His 6 that resulted in a migration pattern that corresponds to a molecular weight of $\sim 440 \mathrm{kDa}$, instead of the predicted molecular weight of $\sim 100 \mathrm{kDa}$ (Figure 3.4B). Also, it is important to note that there is a small population of $\mathrm{T} 7-\mathrm{Vac} 14^{\mathrm{wt}}-\mathrm{His} 6$ that was also found in the fraction that corresponds to $\sim 440 \mathrm{kDa}$ (Figure 3.4A). Although we cannot determine the homomeric state of the Vac14 based the FPLC migration pattern, the results indicated that the purified recombinant T7-Vac14 ${ }^{\mathrm{wt}}$-His 6 exists in both monomeric and multimeric form. 
Differential velocity ultracentrifugation with glycerol gradient was used as an alternative method for identifying the molecular weight of recombinant $\mathrm{T} 7-\mathrm{Vac} 14^{\mathrm{wt}}$-His 6 homomeric complex. Protein separation by velocity ultracentrifugation in a density gradient is based on the effect of frictional force on the protein as it migrates through the different density of glycerol. A larger molecular weight translates into higher frictional force, and migrating through the density gradient at a much slower rate compared to a smaller molecule (124). Recombinant T7-Vac14 ${ }^{\mathrm{wt}}$ His6 was detected in glycerol fractions 1-4 with peak intensity in fraction 2-3. Due to the low resolution of the glycerol gradient, the protein migration pattern generally resembles a broad parabola similar the pattern observed for the protein standards (Figure 3.5A). Therefore, the fraction containing the peak intensity was used to define the location of the majority of protein. Hence, the migration pattern of recombinant $\mathrm{T} 7-\mathrm{Vac} 14^{\mathrm{wt}}$-His6 suggests that the Vac14 complex has a molecular weight of $\sim 200 \mathrm{kDa}$ (corresponding to catalase) (Figure 3.5B). In contrast, the monomeric T7-Vac14 ${ }^{\mathrm{SS}}$-His 6 was detected in fractions1-3, with peak intensity in fraction 2. Although the migration pattern of monomeric T7-Vac14 ${ }^{\mathrm{SS}}$-His6 varies slightly from the $\sim 100 \mathrm{kDa}$ phosphorylase $b$, the slight difference may be attributable to the Stoke's radius of Vac14. Differential velocity ultracentrifugation with glycerol gradient may still be affected by Stoke's radius but to a lesser degree compared to protein separation by FPLC (124). As a consequence, recombinant $\mathrm{T} 7-\mathrm{Vac} 14^{\mathrm{WT}}{ }^{\mathrm{W}}$-His 6 homomeric complex may exist as a homodimer that has a molecular weight of $\sim 200 \mathrm{kDa}$. 


\subsubsection{Vac14 multimer is required for the formation of the Fab1 complex and}

regulation of PtdIns $(3,5) \mathrm{P}_{2}$

Both the yeast and mammalian studies demonstrated that Vac14 acts as a scaffold interacting with Fab1 and Fig4 to form the Fab1 complex. This study also showed that Vac14 most likely self-interacts to form a homodimer complex. However, it is unclear whether this Vac14 complex is essential for interaction with Fig4 and Fab1 to form the Fab1 complex, and in turn to regulate the level of PtdIns(3,5) $\mathrm{P}_{2}$. IP analysis indicates that both monomeric Vac14 ${ }^{S S}$-FLAG and Vac14 ${ }^{C R Y}$-FLAG failed to interact with Fig4 (Figure 3.6). In addition, in the Master thesis by T. Alghamdi, Vac1 $4^{S S}$-FLAG and Vac14 ${ }^{C R Y}$-FLAG also failed to interact with Fab1-myc (116). Together, these data suggest that the formation of a Vac14 homomeric complex is required for its interaction with Fig4 and Fab1 to form the Fab1 complex (Figure 4.2). To investigate if the inability to form the Fab1 complex translates into inefficient synthesis and regulation of $\operatorname{Ptd} \operatorname{Ins}(3,5) \mathrm{P}_{2}$, we next looked at the ability of monomeric Vac14 ${ }^{S S}$-FLAG and $\operatorname{Vac} 14^{N G}$-FLAG to rescue the enlarged vacuolar phenotype observed in vac144 mutant. The enlarged vacuolar phenotype in vac144 is associated with deficient in the level of $\operatorname{PtdIns}(3,5) \mathrm{P}_{2}$. Fluorescence microscopy results showed that expression of monomeric Vac14 ${ }^{S S}$-FLAG and Vacl $^{N G}$-FLAG in vacl44 retained the enlarged vacuolar phenotype, while only in the presence of the wild-type Vac14 was able to rescue the vac14A phenotype (Figure 3.7A). In addition, hyperosmotic stress is known to hyperactivate Fab1 for the synthesis of $\operatorname{PtdIns}(3,5) \mathrm{P}_{2}$, and results in the increased in vacuolar fission. Instead of vacuolar fission observed in vac144 expressing wild-type Vac14, vacl44 expressing monomeric Vacl $4^{S S}$-FLAG and $\mathrm{Vac} 14^{N G}$-FLAG has an enlarged vacuole that appears to have a difficult time forming membrane invaginations (Figure 3.7B). This is perhaps an indication of the vacuole attempting to undergo fission but the $\operatorname{PtdIns}(3,5) \mathrm{P}_{2}$-deficiency may have prevented it from undergoing a full fission event. 


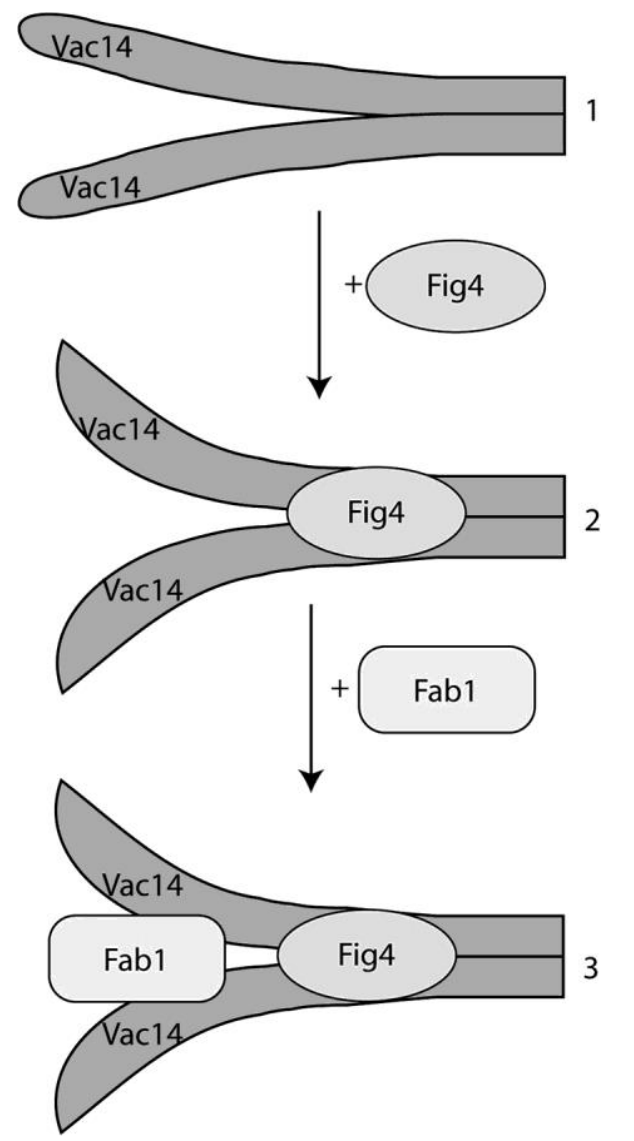

Figure 4. 2 Putative model for the assembly of the Fab1 complex.

Vac14 self-interacts in the C-terminal to form a homodimer (1). The Vac14 homodimer then interacts with Fig4 to form the Vac14-Fig4 complex (2). Formation of the Fig4-Vac14 complex may lead to a conformational change that allows further Vac14 interaction with Fab1 (3) (from 111). 


\subsection{PtdIns $(3,5) \mathrm{P}_{2}$ is not required for the regulation of steady-state vacuolar and lysosomal acidification}

The inability to efficiently synthesize $\operatorname{Ptd} \operatorname{Ins}(3,5) \mathrm{P}_{2}$ in yeast has been thought to result in vacuolar/lysosomal acidification defects $(5,86-88)$. Yeast cells containing mutants or deletions of regulators involved in the synthesis of PtdIns $(3,5) \mathrm{P}_{2}$ do not accumulate the vacuolar acidic marker, quinacrine $(82,89)$. In contrast, the status of the lysosomal $\mathrm{pH}$ in mammalian cells deficient in $\operatorname{PtdIns}(3,5) \mathrm{P}_{2}$ remains unclear. This is due to contradicting studies, with one indicating that the inhibition of PtdIns(3,5) $\mathrm{P}_{2}$ synthesis by MF4 did not affect late endocytic accumulation of the acidic marker, acridine orange, wAnother study suggests that inhibition of $\operatorname{PtdIns}(3,5) \mathrm{P}_{2}$ synthesis by YM201636, an inhibitor of PIKfyve resulted in loss of the acidotrophic lysotracker colocalization with late endocytic markers. $(98,125,126)$. However, the methods used in these studies were all qualitative assays. The actual $\mathrm{pH}$ status of the vacuolar/lysosomal lumen in $\operatorname{Ptd} \operatorname{Ins}(3,5) \mathrm{P}_{2}$-deficient cells was never quantified in these studies. The current study reports that both the yeast vacuole and mammalian lysosome remain acidic in $\operatorname{Ptd} \operatorname{Ins}(3,5) \mathrm{P}_{2}$-deficient cells.

\subsubsection{PtdIns $(3,5) \mathrm{P}_{2}$ is not required for vacuolar acidification under basal conditions in yeast}

Consistent with previous studies, we confirmed that quinacrine does not accumulate in vacuoles deficient in PtdIns(3,5) $\mathrm{P}_{2}$ (Figure 3.8). However, our quantitative analyses of vacuolar $\mathrm{pH}$ contradict the quinacrine assay, and instead suggest that the level of $\operatorname{PtdIns}(3,5) \mathrm{P}_{2}$ does not affect vacuolar acidification under basal conditions. To measure vacuolar $\mathrm{pH}$, we took advantage of the vacuolar accumulation of pHluorin fused to Mup1, in the presence of methionine (Figure 3.9). The fluorescence property of pHluorin is quenched when fully protonated at an acidic $\mathrm{pH}$ and its intensity increases as it is deprotonated with increasing $\mathrm{pH}$. Changes in fluorescence intensity were recorded when yeast cells were subjected to varying extracellular $\mathrm{pH}$ values. Consistent with the model that $v p h 1 \Delta$ have a more alkaline vacuolar $\mathrm{pH}$ due to a non-functional V-ATPase, $v p h 1 \Delta$ 
displayed a significantly reduced change in pHluorin intensity compared to wild-type when equilibrated to pH7.5 (Figure 3.10B). Interestingly, the vacuolar pHluorin in fablA mutant appeared to be quenched to a similar extend to wild-type (Figure 3.10A). CCCP equilibration of vacuolar lumen to $\mathrm{pH} 7.5$ resulted in a large but similar change in fluorescence intensity in both wild-type and fab1 1 mutant (Figure 3.10B). This suggests that the vacuole in fabl 14 mutant has a similar luminal $\mathrm{pH}$ to wild-type.

Furthermore, using fluorimetry analysis of vacuolar $\mathrm{pH}$ with cDCFDA, fab14 and vac144 mutants were measured to have a similar acidic vacuolar $\mathrm{pH}$ to wild-type of $\sim \mathrm{pH} 4.9$. In addition to the mutants deficient in PtdIns(3,5) $\mathrm{P}_{2}$ (fab1 $\Delta$ and vac144), we showed that the $\sim 20$-fold increase in PtdIns(3,5) $\mathrm{P}_{2}$ in atg184 also did not affect the vacuolar $\mathrm{pH}$ (Figure 3.14A). Together with the Mup1-pHluorin assay, the different level of PtdIns(3,5) $\mathrm{P}_{2}$ does not appear to affect proper vacuolar acidification.

In addition, the inhibition or deletion of a functional V-ATPase, by introducing ConA or $v p h 1 \Delta$, respectively, abolished the acidic vacuolar $\mathrm{pH}$ observed in fabl 1 (Figure 3.14-4.8). These data suggest that the observed acidic vacuolar $\mathrm{pH}$ in cells deficient in $\operatorname{PtdIns}(3,5) \mathrm{P}_{2}$ is due to a functional V-ATPase. However, a recent study indicates that $\operatorname{PtdIns}(3,5) \mathrm{P}_{2}$ interacts with and increases the activity of V-ATPase in-vitro (94). This was supported by the evidence that there is an increase in the recruitment and activity of V-ATPase with increased PtdIns $(3,5) \mathrm{P}_{2}$ during both hyperosmotic shock and glucose-deprivation (94). Although the results presented in this study appear to contradict the role of PtdIns(3,5) $\mathrm{P}_{2}$ in V-ATPase acidification of the vacuole suggested by $\mathrm{Li}$ et al., it is important to note that these studies were done under stress conditions and that the vacuolar $\mathrm{pH}$ was not quantified. In this study, the observed acidic vacuolar $\mathrm{pH}$ in $\mathrm{PtdIns}(3,5) \mathrm{P}_{2-}$ deficient cells was measured under basal condition. In addition, other immunofluorescence studies 
also showed that Vph1 remained localized to the vacuolar membrane in $\operatorname{PtdIns}(3,5) \mathrm{P}_{2}$-deficient cells $(89,95)$. Taken together, we speculate that under basal condition, there is minimal level of V-ATPase activity present on vacuolar membrane that may be enough for sustaining the observed acidic vacuolar $\mathrm{pH}$ in $\operatorname{PtdIns}(3,5) \mathrm{P}_{2}$-deficient cells. In contrast, $\operatorname{PtdIns}(3,5) \mathrm{P}_{2}$ may be necessary to interact with the V-ATPase to increase its recruitment and activity on vacuolar membrane in response to stressful conditions such as glucose deprivation and exposure to a hyperosmotic environment. Indeed, the current study showed that under hyperosmotic stress, PtdIns $(3,5) \mathrm{P}_{2}$ deficient fabld mutant was more susceptible to alkalinization of the vacuole compared to wildtype (Figure 3.16). Furthermore, there may be additional regulatory pathways to compensate for the potentially low V-ATPase activity in $\operatorname{PtdIns}(3,5) \mathrm{P}_{2}$-deficient cells. A variety of counter-ion channels and exchangers localized on the vacuolar membrane may help to establish the observed acidic vacuole (24-26).

It is uncertain why despite the observed acidic vacuoles in fabl $1 \Delta$ mutant, the vacuole failed to accumulate quinacrine. A possibility is that quinacrine may require another factor other than PtdIns(3,5) $\mathrm{P}_{2}$ for its vacuolar localization. Besides being used as a vacuolar marker, quinacrine has been used as a drug to treat malaria and possibly an anti-cancer agent (127). Quinacrine was found to have affinity to and binding to nucleotides to mediate its therapeutic activities $(127,128)$. Studies have shown that there are ATP transporters on the lysosomal membrane that mediate storage of lysosomal ATP, and that quinacrine was identified in ATP containing lysosomal vesicles $(129,130)$. In addition, quinacrine was also shown to have affinity to polyphosphates (polyP), a long chain of phosphate molecules for the regulation of free phosphate $\left(\mathrm{P}_{\mathrm{i}}\right)$ levels $(131)$. The yeast vacuole is the main reservoir for cellular polyP, therefore, it is not surprising that 
vacuolar ATP or polyP may be factors affecting quinacrine accumulation in the PtdIns $(3,5) \mathrm{P}_{2-}$ deficient vacuoles.

\subsubsection{The lysosomal $\mathrm{pH}$ remains acidic in the presence of PIKfyve inhibitors}

Studies of PtdIns(3,5)P 2 synthesis by PIKfyve often employs the use of PIKfyve inhibitors, such as apilimod and MF4 $(98,132)$. Mammalian cells inhibited with either apilimod or MF4 retained an acidic lysosome comparable to control (Figure 3.18B). Inhibition of PIKfyve by apilimod was extended to 4-hrs to exclude the possibility that the observed acidic lysosomes in 1hr apilimod treated cells were due to lysosomal acidification before treatment of inhibitors. However, even after prolonged inhibition by apilimod, the lysosomal $\mathrm{pH}$ remained acidic. Inhibition of the V-ATPase by ConA effectively alkalinized the lysosomes in control and apilimod-treated cells (Figure 3.18B). In addition, we showed that the acidotrophic lysostracker accumulated in membranes of enlarged late endocytic structures in the presence of the PtdIns(3,5) $\mathrm{P}_{2}$ inhibitor, Apilimod (114). These results from the mammalian model in this study is consistent with the yeast model, together suggest that $\operatorname{Ptd} \operatorname{Ins}(3,5) \mathrm{P}_{2}$ is not necessary for basal acidification of the vacuole or lysosome. 
Chapter 5: Conclusions 


\subsection{The Vac14 homodimer complex is required for the regulation of $\operatorname{PtdIns}(3,5) \mathrm{P}_{2}$}

Based on the current study and together with the published literature, we postulate that the yeast Vac14 directly interacts with another copy of Vac14 through its C-terminus (residues 560653) to form a homodimer. The formation of this Vac14 homodimer with molecular weight 200kDa, appears to be upstream of its interaction with Fig4 in the cytosol. Formation of this Vac14 dimer-Fig4 heterocomplex then interacts with Fab1 to form the Fab1 complex on the vacuolar membrane (Figure 4.2). Inability to form the Vac14 homodimer results in the loss of the Fab1 complex, and therefore leads to the inefficient synthesis of $\operatorname{PtdIns}(3,5) \mathrm{P}_{2}$.

\subsection{PtdIns $(3,5) \mathrm{P}_{2}$ is not required for the regulation of steady-state vacuolar and lysosomal acidification}

The current study used a number of quantitative assays to characterize the vacuolar and lysosomal $\mathrm{pH}$ in the PtdIns $(3,5) \mathrm{P}_{2}$-deficient conditions. Results presented indicate that both the $\operatorname{PtdIns}(3,5) \mathrm{P}_{2}$-deficient vacuoles and lysosomes remain acidic with a $\mathrm{pH}$ of $\sim 4.8-4.9$. In addition, the observed acidity is found to be dependent on a functional V-ATPase. Contradicting past beliefs, we proposed that under basal condition, $\operatorname{PtdIns}(3,5) \mathrm{P}_{2}$ doesnot have a role in maintaining the steady-state vacuolar/lysosomal $\mathrm{pH}$. However, $\operatorname{Ptd} \operatorname{Ins}(3,5) \mathrm{P}_{2}$ may come into play during stress conditions, such as hyperosmotic shock, to increase activation of V-ATPase. 
Chapter 6: Future directions 


\subsection{Identify the factor that affects vacuolar accumulation of quinacrine}

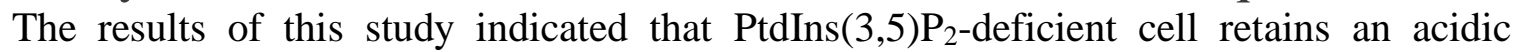

vacuole. Therefore, the acidic environment may not be the only explanation for vacuolar accumulation of quinacrine as previously thought. Quinacrine is known to bind polyphosphates (polyP), a long chain of phosphate molecules that is found in both prokaryotic and eukaryotic organisms. PolyP is mainly found in the vacuolar lumen of yeast and is important for a variety of cellular functions but mainly serves as the storage of energy $(133,134)$. Hydrolysis of polyP releases free phosphates $\left(\mathrm{P}_{\mathrm{i}}\right)$ which can be use for the synthesis of ATP. It was previously shown that pho84, which is deleted for a cyclin known for the regulation of intracellular phosphates, has both a higher polyP concentration and an increase in the intensity of vacuolar quinacrine staining (135). This increase in signal intensity of vacuolar quinacrine was suggested to correlate to a higher vacuolar quinacrine concentration. The authors suggest that the increase in vacuolar quinacrine concentration is due to a more acidic vacuolar lumen, based on the assumption that the accumulation of quinacrine is dependent on an acidic vacuole (135). However, taking the current study into consideration, the change in vacuolar quinacrine intensity in pho 84 may be attributable to the increase in polyP instead. To investigate whether polyP has a role in the loss of vacuolar accumulation of quinacrine in $\operatorname{Ptd} \operatorname{Ins}(3,5) \mathrm{P}_{2}$-deficient yeast, the next step is to quantify the level of polyP in fablu.

Cellular polyP can be extracted and quantified using the malachite green assay $(136,137)$. Extracted polyP is first digested into $\mathrm{P}_{\mathrm{i}}$ by exopolyphosphatase. The malachite green then reacts with $\mathrm{P}_{\mathrm{i}}$ to form a green complex that can be measured by a spectrophotometer at $600-660 \mathrm{~nm}$. In addition, Raman microspectroscopy can be use to measure vacuolar polyP levels in real-time (138). Raman microspectroscopy is the study of scattered photons as they interact with surrounding molecules. The dispersion pattern of the scattered photons depends on the distinct 
vibration of the molecule, while the signal intensity corresponds to the concentration of the molecule (139). Therefore, the malachite green assay and Raman microspectrocopy can both be employed to study the concentration of polyP in $\operatorname{Ptd} \operatorname{Ins}(3,5) \mathrm{P}_{2}$-deficient yeast.

\subsection{Characterize the interaction of recombinant human Vac14 (ArPIKfyve) containing point mutations recently identified in patients with neurodegenerative diseases}

$\operatorname{PtdIns}(3,5) \mathrm{P}_{2}$ dysfunction is believed to be linked to several human neuropathologies, such

as Charcot-Marie-Tooth disease type 4J (CMT4J) and amyotrophic lateral sclerosis subtype 11 (3, 4). Patients with CMT4J were found to carry a mutated allele of FIG4/SAC3 that prevents interaction of Fig4 with Vac14/ArPIKfyve $(140,141)$. A mouse model of CMT4J indicates that expression of the mutant Fig4 results in a significant reduction in the level of PtdIns(3,5) $\mathrm{P}_{2}$ and neurodegeneration in the central and peripheral nervous systems (4).

Recently, M. Meisler's lab at the University of Michigan has identified two mutant alleles of ArPIKfyve in human patients with neurological diseases (not published). Each allele contains a single mutation, found in the mutated region of the $v a c 14^{S I A}$ mutant described in this study that changed the hydrophobic-hydrophilic nature of this region (Chapter 3; Figure 3.2). The current study (Chapter 5.1) proposed that Vac14 self-interaction is necessary for the regulation of $\operatorname{PtdIns}(3,5) \mathrm{P}_{2}$. Therefore, the expression of the mutant alleles of ArPIKfyve may disrupt its ability to self-interact and cause PtdIns $(3,5) \mathrm{P}_{2}$ dysregulation, which could lead to neurodegeneration. To investigate this, we can modify the in-vitro recombinant interaction assay and test for the recombinant human Vac14 self-interaction with the two mutant alleles of human Vac14. Identifying the loss of human Vac14 homodimerization is significant for supporting the idea that expression of the mutant alleles in human patients leading to neurodegeneration may be due to its inability to form the Vac14 homodimer complex for the synthesis of PtdIns(3,5) $\mathrm{P}_{2}$. 


\section{References}


1. Ikonomov, O. C., Sbrissa, D., and Shisheva, a (2001) Mammalian cell morphology and endocytic membrane homeostasis require enzymatically active phosphoinositide 5-kinase PIKfyve. J. Biol. Chem. 276, 26141-7

2. Botelho, R. J., Efe, J. A., Teis, D., and Emr, S. D. (2008) Assembly of a Fab1 phosphoinositide kinase signaling complex requires the Fig4 phosphoinositide phosphatase. Mol. Biol. Cell. 19, 4273-86

3. Chow, C. Y., Landers, J. E., Bergren, S. K., Sapp, P. C., Grant, A. E., Jones, J. M., Everett, L., Lenk, G. M., McKenna-Yasek, D. M., Weisman, L. S., Figlewicz, D., Brown, R. H., and Meisler, M. H. (2009) Deleterious variants of FIG4, a phosphoinositide phosphatase, in patients with ALS. Am. J. Hum. Genet. 84, 85-8

4. Chow, C. Y., Zhang, Y., Dowling, J. J., Jin, N., Adamska, M., Shiga, K., Szigeti, K., Shy, M. E., Li, J., Zhang, X., James, R., Weisman, L. S., and Meisler, M. H. (2007) Mutation of FIG4 causes neurodegeneration in the pale tremor mouse and patients with CMT4J. Nature. 448, 68-72

5. Li, S. C., and Kane, P. M. (2010) The yeast lysosome-like vacuole: Endpoint and crosscroads. Biochim Biophs Acta. 1793, 650-663

6. Saftig, P., and Klumperman, J. (2009) Lysosome biogenesis and lysosomal membrane proteins: trafficking meets function. Nat. Rev. Mol. Cell Biol. 10, 623-35

7. Stipanuk, M. H. (2009) Macroautophagy and its role in nutrient homeostasis. Nutr. Rev. 67, 677-689

8. Sekito, T., Fujiki, Y., Ohsumi, Y., and Kakinuma, Y. (2008) Novel families of vacuolar 
amino acid transporters. IUBMB Life. 60, 519-25

9. Simm, C., Lahner, B., Salt, D., LeFurgey, A., Ingram, P., Yandell, B., and Eide, D. J. (2007) Saccharomyces cerevisiae vacuole in zinc storage and intracellular zinc distribution. Eukaryot. Cell. 6, 1166-77

10. Shimazu, M., Sekito, T., Akiyama, K., Ohsumi, Y., and Kakinuma, Y. (2005) A family of basic amino acid transporters of the vacuolar membrane from Saccharomyces cerevisiae. J. Biol. Chem. 280, 4851-7

11. Ballabio, A., and Gieselmann, V. (2009) Lysosomal disorders: From storage to cellular damage. Biochim. Biophys. Acta - Mol. Cell Res. 1793, 684-696

12. Froehlich, S. J., Lackerbauer, C. A., Rudolph, G., Rémi, J., Noachtar, S., Heppt, W. J., Cryer, A., Zenner, H.-P., Niller, H. H., Schwarzmann, F., Wolf, H., Frieling, T., Leung, A. K. C., Wallgren-Pettersson, C., Goebel, H. H., Jouret, F., Devuyst, O., Attanasio, M., Hildebrandt, F., Jha, V., Chugh, K. S., Marinaki, A. M., Simmonds, H. A., Zhou, X., Frohlich, E. D., Tesar, V., Jalanko, H., Oji, V., Traupe, H., Danek, A., Angelini, C., Kaufmann, D., Kaufmann, D., Weller, M., Kornhuber, J., Vincent, A., Newsom-Davis, J., Mole, S., Williams, R., Baets, J., Hanemann, C. O., Jonghe, P., Holt, I., Klopstock, T., Zeviani, M., Ley, K., Smith, E., Shiohara, M., Leung, A. K. C., Robson, W. L. M., Brokalaki, E. I., Grabbe, S., Loquai, C., Nohr, D., Nohr, D., Gallinat, J., Schuchman, E. H., Desnick, R. J., Zeitz, C., Mizutani, S., Orange, J. S., Weiss, J. P., Blaivas, J. G., Robson, W. L. M., Leung, A. K. C., Ma, L. K., Ma, P. T. S., Leung, A. K. C., Schwarz, P. E. H., Li, J., LeRoith, D., Kure, S., Tada, K., Utikal, J., Gratchev, A., Goerdt, S., Senzolo, M., Piovesana, E., Okolicsanyi, L., Burroughs, A. K., Sistermans, E. A., Engel, J., Fischer, 
M. D., Berger, W., Tozzi, M. G., Micheli, V., Leung, A. K. C., and Robson, W. L. M. (2009) Niemann-Pick Disease Types A and B. in Encyclopedia of Molecular Mechanisms of Disease, pp. 1479-1480, Springer Berlin Heidelberg, Berlin, Heidelberg, 10.1007/9783-540-29676-8_1286

13. Patterson, M. (1993) Niemann-Pick Disease Type C, University of Washington, Seattle, [online] http://www.ncbi.nlm.nih.gov/pubmed/20301473 (Accessed August 22, 2016)

14. Yamashiro, D. J., and Maxfield, F. R. (1987) Acidification of morphologically distinct endosomes in mutant and wild-type Chinese hamster ovary cells. J. Cell Biol. 105, 272333

15. Sipe, D. M., and Murphy, R. F. (1987) High-resolution kinetics of transferrin acidification in BALB/c 3T3 cells: exposure to $\mathrm{pH} 6$ followed by temperature-sensitive alkalinization during recycling. Proc. Natl. Acad. Sci. U. S. A. 84, 7119-23

16. Johnson, M. B., Chen, J., Murchison, N., Green, F. A., and Enns, C. A. (2007) Transferrin receptor 2: evidence for ligand-induced stabilization and redirection to a recycling pathway. Mol. Biol. Cell. 18, 743-54

17. Dautry-varsat, A., Ciechanover, A., and Lodish, H. F. (1983) pH and the recycling of transferrin during receptor- mediated endocytosis. Proc. Natl. Acad. Sci. USA. 80, 22582262

18. Raiborg, C., Bache, K. G., Gillooly, D. J., Madshus, I. H., Stang, E., and Stenmark, H. (2002) Hrs sorts ubiquitinated proteins into clathrin-coated microdomains of early endosomes. Nat. Cell Biol. 4, 394-8 
19. Raiborg, C., Malerød, L., Pedersen, N. M., and Stenmark, H. (2008) Differential functions of Hrs and ESCRT proteins in endocytic membrane trafficking. Exp. Cell Res. 314, 80113

20. Kim, J. H., Johannes, L., Goud, B., Antony, C., Lingwood, C. A., Daneman, R., and Grinstein, S. (1998) Noninvasive measurement of the $\mathrm{pH}$ of the endoplasmic reticulum at rest and during calcium release. Proc. Natl. Acad. Sci. U. S. A. 95, 2997-3002

21. Kim, J. H., Lingwood, C. A., Williams, D. B., Furuya, W., Manolson, M. F., and Grinstein, S. (1996) Dynamic measurement of the $\mathrm{pH}$ of the Golgi complex in living cells using retrograde transport of the verotoxin receptor. J. Cell Biol. 134, 1387-99

22. Llopis, J., McCaffery, J. M., Miyawaki, A., Farquhar, M. G., and Tsien, R. Y. (1998) Measurement of cytosolic, mitochondrial, and Golgi $\mathrm{pH}$ in single living cells with green fluorescent proteins. Proc. Natl. Acad. Sci. U. S. A. 95, 6803-8

23. Nozaki, Y., and Tanford, C. (1981) Proton and hydroxide ion permeability of phospholipid vesicles. Proc. Natl. Acad. Sci. U. S. A. 78, 4324-4328

24. Grabe, M., and Oster, G. (2001) Regulation of organelle acidity. J. Gen. Physiol. 117, 329-44

25. Steinberg, B. E., Huynh, K. K., Brodovitch, A., Jabs, S., Stauber, T., Jentsch, T. J., and Grinstein, S. (2010) A cation counterflux supports lysosomal acidification. J. Cell Biol. 189, $1171-86$

26. Cain, C. C., Sipe, D. M., and Murphy, R. F. (1989) Regulation of endocytic pH by the Na+,K+-ATPase in living cells. Proc. Natl. Acad. Sci. U. S. A. 86, 544-8 
27. HAZEL, H. B., KIELLAND-BRANDT, M. C., and WINTHER, J. R. (1992)

Autoactivation of proteinase A initiates activation of yeast vacuolar zymogens. Eur. $J$. Biochem. 207, 277-283

28. Rupp, S., Hirsch, H. H., and Wolf, D. H. (1991) Biogenesis of the yeast vacuole (lysosome) Active site mutation in the vacuolar aspartate proteinase yscA blocks maturation of vacuolar proteinases. FEBS Lett. 293, 62-66

29. Sørensen, S. O., van den Hazel, H. B., Kielland-Brandt, M. C., and Winther, J. R. (1994) $\mathrm{pH}$-dependent processing of yeast procarboxypeptidase $\mathrm{Y}$ by proteinase $\mathrm{A}$ in vivo and in vitro. Eur. J. Biochem. 220, 19-27

30. Benes, P., Vetvicka, V., and Fusek, M. (2008) Cathepsin D--many functions of one aspartic protease. Crit. Rev. Oncol. Hematol. 68, 12-28

31. Conner, G. E., and Richo, G. (1992) Isolation and characterization of a stable activation intermediate of the lysosomal aspartyl protease cathepsin D. Biochemistry. 31, 1142-7

32. Gieselmann, V., Hasilik, A., and von Figura, K. (1985) Processing of human cathepsin D in lysosomes in vitro. J. Biol. Chem. 260, 3215-20

33. Conibear, E., and Stevens, T. H. (1998) Multiple sorting pathways between the late Golgi and the vacuole in yeast. Biochim. Biophys. Acta - Mol. Cell Res. 1404, 211-230

34. Krämer, H. (2013) Route to destruction: autophagosomes SNARE lysosomes. J. Cell Biol. 201, 495-7

35. Jahn, R., Lang, T., and Südhof, T. C. (2003) Membrane Fusion. Cell. 112, 519-533 
36. Koumandou, V. L., Dacks, J. B., Coulson, R. M. R., and Field, M. C. (2007) Control systems for membrane fusion in the ancestral eukaryote; evolution of tethering complexes and SM proteins. BMC Evol. Biol. 7, 29

37. Nichols, B. J., Ungermann, C., Pelham, H. R., Wickner, W. T., and Haas, A. (1997) Homotypic vacuolar fusion mediated by t- and v-SNAREs. Nature. 387, 199-202

38. Ungermann, C., Nichols, B. J., Pelham, H. R., and Wickner, W. (1998) A vacuolar v-tSNARE complex, the predominant form in vivo and on isolated vacuoles, is disassembled and activated for docking and fusion. J. Cell Biol. 140, 61-9

39. Wada, Y., Nakamura, N., Ohsumi, Y., and Hirata, A. (1997) Vam3p, a new member of syntaxin related protein, is required for vacuolar assembly in the yeast Saccharomyces cerevisiae. J. Cell Sci. 110 ( Pt 1, 1299-306

40. Leabu, M. (2006) Membrane fusion in cells: molecular machinery and mechanisms. $J$. Cell. Mol. Med. 10, 423-7

41. Pryor, P. R., Mullock, B. M., Bright, N. A., Gray, S. R., and Luzio, J. P. (2000) The role of intraorganellar $\mathrm{Ca}(2+)$ in late endosome-lysosome heterotypic fusion and in the reformation of lysosomes from hybrid organelles. J. Cell Biol. 149, 1053-62

42. Bakker, A., Webster, P., Jacob, W., and Andrews, N. (1997) Homotypic fusion between aggregated lysosomes triggered by elevated [Ca2+]i in fibroblasts. J. Cell Sci. 110, 22272238

43. Cheng, X., Shen, D., Samie, M., and Xu, H. (2010) Mucolipins: Intracellular TRPML1-3 channels. FEBS Lett. 584, 2013-21 
44. Kim, H. J., Soyombo, A. A., Tjon-Kon-Sang, S., So, I., and Muallem, S. (2009) The $\mathrm{Ca}(2+)$ channel TRPML3 regulates membrane trafficking and autophagy. Traffic. 10, $1157-67$

45. Dong, X., Wang, X., Shen, D., Chen, S., Liu, M., Wang, Y., Mills, E., Cheng, X., Delling, M., and Xu, H. (2009) Activating mutations of the TRPML1 channel revealed by prolinescanning mutagenesis. J. Biol. Chem. 284, 32040-52

46. Treusch, S., Knuth, S., Slaugenhaupt, S. A., Goldin, E., Grant, B. D., and Fares, H. (2004) Caenorhabditis elegans functional orthologue of human protein h-mucolipin-1 is required for lysosome biogenesis. Proc. Natl. Acad. Sci. U. S. A. 101, 4483-8

47. Venkatachalam, K., Long, A. A., Elsaesser, R., Nikolaeva, D., Broadie, K., and Montell, C. (2008) Motor deficit in a Drosophila model of mucolipidosis type IV due to defective clearance of apoptotic cells. Cell. 135, 838-51

48. Venugopal, B., Browning, M. F., Curcio-Morelli, C., Varro, A., Michaud, N., Nanthakumar, N., Walkley, S. U., Pickel, J., and Slaugenhaupt, S. A. (2007) Neurologic, gastric, and opthalmologic pathologies in a murine model of mucolipidosis type IV. Am. J. Hum. Genet. 81, 1070-83

49. Cui, J., Kaandorp, J. A., Ositelu, O. O., Beaudry, V., Knight, A., Nanfack, Y. F., and Cunningham, K. W. (2009) Simulating calcium influx and free calcium concentrations in yeast. Cell Calcium. 45, 123-32

50. MacDiarmid, C. W., Gaither, L. A., and Eide, D. (2000) Zinc transporters that regulate vacuolar zinc storage in Saccharomyces cerevisiae. EMBO J. 19, 2845-55 
51. Miyabe, S., Izawa, S., and Inoue, Y. (2001) The Zrc1 is involved in zinc transport system between vacuole and cytosol in Saccharomyces cerevisiae. Biochem. Biophys. Res. Commun. 282, 79-83

52. Ramsay, L. M., and Gadd, G. M. (1997) Mutants of Saccharomyces cerevisiae defective in vacuolar function confirm a role for the vacuole in toxic metal ion detoxification. FEMS Microbiol. Lett. 152, 293-8

53. Ögmundsdóttir, M. H., Heublein, S., Kazi, S., Reynolds, B., Visvalingam, S. M., Shaw, M. K., and Goberdhan, D. C. I. (2012) Proton-assisted amino acid transporter PAT1 complexes with Rag GTPases and activates TORC1 on late endosomal and lysosomal membranes. PLoS One. 7, e36616

54. Thwaites, D. T., and Anderson, C. M. H. (2011) The SLC36 family of proton-coupled amino acid transporters and their potential role in drug transport. Br. J. Pharmacol. 164, $1802-16$

55. Boll, M., Foltz, M., Rubio-Aliaga, I., Kottra, G., and Daniel, H. (2002) Functional characterization of two novel mammalian electrogenic proton-dependent amino acid cotransporters. J. Biol. Chem. 277, 22966-73

56. Finbow, M. E., and Harrison, M. a (1997) The vacuolar H+-ATPase: a universal proton pump of eukaryotes. Biochem. J. 324 ( Pt 3, 697-712

57. Sonnenburg, J. L. (1996) Resolution of Subunit Interactions and Cytoplasmic Subcomplexes of the Yeast Vacuolar Proton-translocating ATPase. J. Biol. Chem. 271, $10397-10404$ 
58. Hirata, R., Ohsumk, Y., Nakano, A., Kawasaki, H., Suzuki, K., and Anraku, Y. (1990) Molecular structure of a gene, VMA1, encoding the catalytic subunit of $\mathrm{H}(+)$ translocating adenosine triphosphatase from vacuolar membranes of Saccharomyces cerevisiae. J. Biol. Chem. 265, 6726-33

59. Uchida, E., Ohsumi, Y., and Anraku, Y. (1988) Characterization and function of catalytic subunit alpha of H+-translocating adenosine triphosphatase from vacuolar membranes of Saccharomyces cerevisiae. A study with 7-chloro-4-nitrobenzo-2-oxa-1,3-diazole. J. Biol. Chem. 263, 45-51

60. Forgac, M. (2007) Vacuolar ATPases: rotary proton pumps in physiology and pathophysiology. Nat. Rev. Mol. Cell Biol. 8, 917-29

61. Wang, Y., Toei, M., and Forgac, M. (2008) Analysis of the membrane topology of transmembrane segments in the C-terminal hydrophobic domain of the yeast vacuolar ATPase subunit a (Vph1p) by chemical modification. J. Biol. Chem. 283, 20696-702

62. Parra, K. J., and Kane, P. M. (1998) Reversible association between the V1 and V0 domains of yeast vacuolar H+-ATPase is an unconventional glucose-induced effect. Mol. Cell. Biol. 18, 7064-7074

63. Tarsio, M., Zheng, H., Smardon, A. M., Martínez-Muñoz, G. A., and Kane, P. M. (2011) Consequences of loss of Vph1 protein-containing vacuolar ATPases (V-ATPases) for overall cellular pH homeostasis. J. Biol. Chem. 286, 28089-96

64. Kane, P. M. (2000) Regulation of V-ATPases by reversible disassembly. FEBS Lett. 469, $137-141$ 
65. Li, S. C., Diakov, T. T., Rizzo, J. M., and Kane, P. M. (2012) Vacuolar H+-ATPase works in parallel with the HOG pathway to adapt Saccharomyces cerevisiae cells to osmotic stress. Eukaryot. Cell. 11, 282-91

66. Wenk, M. R., and De Camilli, P. (2004) Protein-lipid interactions and phosphoinositide metabolism in membrane traffic: insights from vesicle recycling in nerve terminals. Proc. Natl. Acad. Sci. U. S. A. 101, 8262-9

67. Shulga, Y. V, Anderson, R. A., Topham, M. K., and Epand, R. M. (2012) Phosphatidylinositol-4-phosphate 5-kinase isoforms exhibit acyl chain selectivity for both substrate and lipid activator. J. Biol. Chem. 287, 35953-63

68. Pettitt, T. R., Martin, A., Horton, T., Liossis, C., Lord, J. M., and Wakelam, M. J. O. (1997) Diacylglycerol and Phosphatidate Generated by Phospholipases C and D, Respectively, Have Distinct Fatty Acid Compositions and Functions: PHOSPHOLIPASE D-DERIVED DIACYLGLYCEROL DOES NOT ACTIVATE PROTEIN KINASE C IN PORCINE AORTIC ENDOTHELIAL CELLS. J. Biol. Chem. 272, 17354-17359

69. Di Paolo, Gilbert \& De Camilli, P. (2006) Phosphoinositides in cell regulation and membrane dynamics. Nature. 10.1038

70. Ikonomov, O. C., Sbrissa, D., and Shisheva, A. (2006) Localized PtdIns 3,5-P2 synthesis to regulate early endosome dynamics and fusion. Am. J. Physiol. Cell Physiol. 291, C393404

71. Burman, C., and Ktistakis, N. T. (2010) Regulation of autophagy by phosphatidylinositol 3-phosphate. FEBS Lett. 584, 1302-12 
72. Stenmark, H., Aasland, R., and Driscoll, P. C. (2002) The phosphatidylinositol 3phosphate-binding FYVE finger. FEBS Lett. 513, 77-84

73. Aasland, R. (1996) Endosomal Localization of the Autoantigen EEA1 Is Mediated by a Zinc-binding FYVE Finger. J. Biol. Chem. 271, 24048-24054

74. Lawe, D. C. (2000) The FYVE Domain of Early Endosome Antigen 1 Is Required for Both Phosphatidylinositol 3-Phosphate and Rab5 Binding. CRITICAL ROLE OF THIS DUAL INTERACTION FOR ENDOSOMAL LOCALIZATION. J. Biol. Chem. 275, $3699-3705$

75. Lawe, D. C., Chawla, A., Merithew, E., Dumas, J., Carrington, W., Fogarty, K., Lifshitz, L., Tuft, R., Lambright, D., and Corvera, S. (2002) Sequential roles for phosphatidylinositol 3-phosphate and Rab5 in tethering and fusion of early endosomes via their interaction with EEA1. J. Biol. Chem. 277, 8611-7

76. Horazdovsky, B. F., Davies, B. A., Seaman, M. N., McLaughlin, S. A., Yoon, S., and Emr, S. D. (1997) A sorting nexin-1 homologue, Vps5p, forms a complex with Vps17p and is required for recycling the vacuolar protein-sorting receptor. Mol. Biol. Cell. 8, $1529-41$

77. Yu, J. W., and Lemmon, M. A. (2001) All phox homology (PX) domains from Saccharomyces cerevisiae specifically recognize phosphatidylinositol 3-phosphate. $J$. Biol. Chem. 276, 44179-84

78. Prehoda, K. E., and Lim, W. A. (2001) The double life of PX domains. Nat. Struct. Biol. $8,570-2$ 
79. Ellson, C. D., Andrews, S., Stephens, L. R., and Hawkins, P. T. (2002) The PX domain: a new phosphoinositide-binding module. J. Cell Sci. 115, 1099-105

80. Whiteford, C. C., Brearley, C. A., and Ulug, E. T. (1997) Phosphatidylinositol 3,5bisphosphate defines a novel PI 3-kinase pathway in resting mouse fibroblasts. Biochem. J. 323, 597-601

81. Dove, S. K., Cooke, F. T., Douglas, M. R., Sayers, L. G., Parker, P. J., and Michell, R. H. (1997) Osmotic stress activates phosphatidylinositol-3,5-bisphosphate synthesis. Nature. 390, $187-92$

82. Bonangelino, C. J., Nau, J. J., Duex, J. E., Brinkman, M., Wurmser, A. E., Gary, J. D., Emr, S. D., and Weisman, L. S. (2002) Osmotic stress-induced increase of phosphatidylinositol 3,5-bisphosphate requires Vac14p, an activator of the lipid kinase Fab1p. J. Cell Biol. 156, 1015-28

83. Dove, S. K., Piper, R. C., McEwen, R. K., Yu, J. W., King, M. C., Hughes, D. C., Thuring, J., Holmes, A. B., Cooke, F. T., Michell, R. H., Parker, P. J., and Lemmon, M. A. (2004) Svp1p defines a family of phosphatidylinositol 3,5-bisphosphate effectors. EMBO J. 23, 1922-33

84. Alghamdi, T. A., Ho, C. Y., Mrakovic, A., Taylor, D., Mao, D., and Botelho, R. J. (2013) Vac14 protein multimerization is a prerequisite step for Fab1 protein complex assembly and function. J. Biol. Chem. 288, 9363-72

85. Sbrissa, D., and Shisheva, A. (2005) Acquisition of unprecedented phosphatidylinositol 3,5-bisphosphate rise in hyperosmotically stressed 3T3-L1 adipocytes, mediated by 
ArPIKfyve-PIKfyve pathway. J. Biol. Chem. 280, 7883-9

86. Odorizzi, G., Babst, M., and Emr, S. D. (1998) Fab1p PtdIns(3)P 5-Kinase Function Essential for Protein Sorting in the Multivesicular Body. Cell. 95, 847-858

87. Rusten, T. E., Rodahl, L. M. W., Pattni, K., Englund, C., Samakovlis, C., Dove, S., Brech, A., and Stenmark, H. (2006) Fab1 phosphatidylinositol 3-phosphate 5-kinase controls trafficking but not silencing of endocytosed receptors. Mol. Biol. Cell. 17, 3989-4001

88. Rutherford, A. C., Traer, C., Wassmer, T., Pattni, K., Bujny, M. V, Carlton, J. G., Stenmark, H., and Cullen, P. J. (2006) The mammalian phosphatidylinositol 3-phosphate 5-kinase (PIKfyve) regulates endosome-to-TGN retrograde transport. J. Cell Sci. 119, 3944-57

89. Gary, J. D., Wurmser, A. E., Bonangelino, C. J., Weisman, L. S., and Emr, S. D. (1998) Fablp Is Essential for PtdIns ( 3 ) P 5-Kinase Activity and the Homeostasis of Vacuolar Size and Membrane Maintenance. J. Cell Biol. 143, 65-79

90. Friant, S., Pécheur, E.-I., Eugster, A., Michel, F., Lefkir, Y., Nourrisson, D., and Letourneur, F. (2003) Ent3p Is a PtdIns(3,5)P2 Effector Required for Protein Sorting to the Multivesicular Body. Dev. Cell. 5, 499-511

91. Dayam, R. M., Saric, A., Shilliday, R. E., and Botelho, R. J. (2015) The PhosphoinositideGated Lysosomal Ca(2+) Channel, TRPML1, Is Required for Phagosome Maturation. Traffic. 16, 1010-26

92. Li, X., Rydzewski, N., Hider, A., Zhang, X., Yang, J., Wang, W., Gao, Q., Cheng, X., and $\mathrm{Xu}, \mathrm{H}$. (2016) A molecular mechanism to regulate lysosome motility for lysosome 
positioning and tubulation. Nat. Cell Biol. 18, 404-417

93. Dong, X., Shen, D., Wang, X., Dawson, T., Li, X., Zhang, Q., Cheng, X., Zhang, Y., Weisman, L. S., Delling, M., and Xu, H. (2010) PI(3,5)P(2) controls membrane trafficking by direct activation of mucolipin $\mathrm{Ca}(2+)$ release channels in the endolysosome. Nat.

Commun. 1, 38

94. Li, S. C., Diakov, T. T., Xu, T., Tarsio, M., Zhu, W., Couoh-Cardel, S., Weisman, L. S., and Kane, P. M. (2014) The signaling lipid PI(3,5)P2 stabilizes V1-Vo sector interactions and activates the V-ATPase. Mol. Biol. Cell. 25, 1251-62

95. Bonangelino, C. J., Catlett, N. L., and Weisman, L. S. (1997) Vac7p, a novel vacuolar protein, is required for normal vacuole inheritance and morphology. Mol. Cell. Biol. 17, $6847-58$

96. Proikas-Cezanne, T., Takacs, Z., Dönnes, P., and Kohlbacher, O. (2015) WIPI proteins: essential PtdIns3P effectors at the nascent autophagosome. J. Cell Sci. 128, 207-17

97. Yamamoto, A., DeWald, D. B., Boronenkov, I. V, Anderson, R. A., Emr, S. D., and Koshland, D. (1995) Novel PI(4)P 5-kinase homologue, Fab1p, essential for normal vacuole function and morphology in yeast. Mol. Biol. Cell. 6, 525-39

98. de Lartigue, J., Polson, H., Feldman, M., Shokat, K., Tooze, S. A., Urbé, S., and Clague, M. J. (2009) PIKfyve regulation of endosome-linked pathways. Traffic. 10, 883-93

99. Cabezas, A., Pattni, K., and Stenmark, H. (2006) Cloning and subcellular localization of a human phosphatidylinositol 3-phosphate 5-kinase, PIKfyve/Fab1. Gene. 371, 34-41

100. Sbrissa, D., Ikonomov, O. C., and Shisheva, A. (2002) Phosphatidylinositol 3-phosphate- 
interacting domains in PIKfyve. Binding specificity and role in PIKfyve. Endomenbrane localization. J. Biol. Chem. 277, 6073-9

101. Shaw, J. D., Hama, H., Sohrabi, F., DeWald, D. B., and Wendland, B. (2003)

PtdIns(3,5)P2 is required for delivery of endocytic cargo into the multivesicular body. Traffic. 4, 479-90

102. Zeilstra-Ryalls, J., Fayet, O., and Georgopoulos, C. (1991) The universally conserved GroE (Hsp60) chaperonins. Annu. Rev. Microbiol. 45, 301-25

103. Guo, S., Stolz, L. E., Lemrow, S. M., and York, J. D. (1999) SAC1-like domains of yeast SAC1, INP52, and INP53 and of human synaptojanin encode polyphosphoinositide phosphatases. J. Biol. Chem. 274, 12990-5

104. Hughes, W. E., Cooke, F. T., and Parker, P. J. (2000) Sac phosphatase domain proteins. Biochem. J. 350 Pt 2, 337-52

105. Sbrissa, D., Ikonomov, O. C., Fu, Z., Ijuin, T., Gruenberg, J., Takenawa, T., and Shisheva, A. (2007) Core protein machinery for mammalian phosphatidylinositol 3,5-bisphosphate synthesis and turnover that regulates the progression of endosomal transport. Novel Sac phosphatase joins the ArPIKfyve-PIKfyve complex. J. Biol. Chem. 282, 23878-91

106. Rudge, S. A., Anderson, D. M., and Emr, S. D. (2004) Vacuole size control: regulation of PtdIns(3,5)P2 levels by the vacuole-associated Vac14-Fig4 complex, a PtdIns(3,5)P2specific phosphatase. Mol. Biol. Cell. 15, 24-36

107. Duex, J. E., Tang, F., and Weisman, L. S. (2006) The Vac14p-Fig4p complex acts independently of Vac7p and couples PI3,5P2 synthesis and turnover. J. Cell Biol. 172, 
693-704

108. Dove, S. K., McEwen, R. K., Mayes, A., Hughes, D. C., Beggs, J. D., and Michell, R. H. (2002) Vac14 controls PtdIns(3,5)P(2) synthesis and Fab1-dependent protein trafficking to the multivesicular body. Curr. Biol. 12, 885-93

109. Jin, N., Chow, C. Y., Liu, L., Zolov, S. N., Bronson, R., Davisson, M., Petersen, J. L., Zhang, Y., Park, S., Duex, J. E., Goldowitz, D., Meisler, M. H., and Weisman, L. S. (2008) VAC14 nucleates a protein complex essential for the acute interconversion of PI3P and $\mathrm{PI}(3,5) \mathrm{P}(2)$ in yeast and mouse. EMBO J. 27, 3221-34

110. Andrade, M. A., Petosa, C., O’Donoghue, S. I., Müller, C. W., and Bork, P. (2001) Comparison of ARM and HEAT protein repeats. J. Mol. Biol. 309, 1-18

111. Ikonomov, O. C., Sbrissa, D., Dondapati, R., and Shisheva, A. (2007) ArPIKfyve-PIKfyve interaction and role in insulin-regulated GLUT4 translocation and glucose transport in 3T3-L1 adipocytes. Exp. Cell Res. 313, 2404-16

112. Ikonomov, O. C., Sbrissa, D., Fenner, H., and Shisheva, A. (2009) PIKfyve-ArPIKfyveSac3 core complex: contact sites and their consequence for Sac3 phosphatase activity and endocytic membrane homeostasis. J. Biol. Chem. 284, 35794-806

113. Sbrissa, D., Ikonomov, O. C., Fenner, H., and Shisheva, A. (2008) ArPIKfyve homomeric and heteromeric interactions scaffold PIKfyve and Sac3 in a complex to promote PIKfyve activity and functionality. J. Mol. Biol. 384, 766-79

114. Ho, C. Y., Choy, C. H., Wattson, C. A., Johnson, D. E., and Botelho, R. J. (2015) The Fab1/PIKfyve phosphoinositide phosphate kinase is not necessary to maintain the $\mathrm{pH}$ of 
lysosomes and of the yeast vacuole. J. Biol. Chem. 290, 9919-28

115. Longtine, M. S., McKenzie, a, Demarini, D. J., Shah, N. G., Wach, a, Brachat, a, Philippsen, P., and Pringle, J. R. (1998) Additional modules for versatile and economical PCR-based gene deletion and modification in Saccharomyces cerevisiae. Yeast. 14, 95361

116. Alghamdi, T. A. (2012) Functional Characterization Of The VAC14 Self- Interaction Domain. Thesis Diss.

117. Prosser, D. C., Whitworth, K., and Wendland, B. (2011) Quantitative analysis of endocytosis with cytolasmic pHluorin chimeras. Traffic. 11, 1141-1150

118. Menant, A., Barbey, R., and Thomas, D. (2006) Substrate-mediated remodeling of methionine transport by multiple ubiquitin-dependent mechanisms in yeast cells. EMBO J. 25, 4436-47

119. Kasianowicz, J., Benz, R., and McLaughlin, S. (1984) The kinetic mechanism by which CCCP (carbonyl cyanide m-chlorophenylhydrazone) transports protons across membranes. J. Membr. Biol. 82, 179-90

120. Ghoul, M., Pommepuy, M., Moillo-Batt, A., and Cormier, M. (1989) Effect of carbonyl cyanide m-chlorophenylhydrazone on Escherichia coli halotolerance. Appl. Environ. Microbiol. 55, 1040-3

121. Harrison, T. S., Chen, J., Simons, E., and Levitz, S. M. (2002) Determination of the pH of the Cryptococcus neoformans vacuole. Med. Mycol. 40, 329-32

122. Seshacharyulu, P., Pandey, P., Datta, K., and Batra, S. K. (2013) Phosphatase: PP2A 
structural importance, regulation and its aberrant expression in cancer. Cancer Lett. 335, 9-18

123. Hong, P., Koza, S., and Bouvier, E. S. P. (2012) Size-Exclusion Chromatography for the Analysis of Protein Biotherapeutics and their Aggregates. J. Liq. Chromatogr. Relat. Technol. 35, 2923-2950

124. Lodish, H., Berk, A., Zipursky, S. L., Matsudaira, P., Baltimore, D., and Darnell, J. (2000) Purification of Cells and Their Parts. [online] http://www.ncbi.nlm.nih.gov/books/NBK21492/ (Accessed May 31, 2016)

125. Hazeki, K., Uehara, M., Nigorikawa, K., and Hazeki, O. (2013) PIKfyve regulates the endosomal localization of $\mathrm{CpG}$ oligodeoxynucleotides to elicit TLR9-dependent cellular responses. PLoS One. 8, e73894

126. Jefferies, H. B. J., Cooke, F. T., Jat, P., Boucheron, C., Koizumi, T., Hayakawa, M., Kaizawa, H., Ohishi, T., Workman, P., Waterfield, M. D., and Parker, P. J. (2008) A selective PIKfyve inhibitor blocks PtdIns(3,5)P(2) production and disrupts endomembrane transport and retroviral budding. EMBO Rep. 9, 164-70

127. Ehsanian, R., Van Waes, C., and Feller, S. M. (2011) Beyond DNA binding - a review of the potential mechanisms mediating quinacrine's therapeutic activities in parasitic infections, inflammation, and cancers. Cell Commun. Signal. 9, 13

128. Selander, R. K. (1973) Interaction of quinacrine mustard with mononucleotides and polynucleotides. Biochem. J. 131, 749-55

129. Bodin, P., and Burnstock, G. (2001) Evidence that release of adenosine triphosphate from 
endothelial cells during increased shear stress is vesicular. J. Cardiovasc. Pharmacol. 38, $900-8$

130. Cao, Q., Zhao, K., Zhong, X. Z., Zou, Y., Yu, H., Huang, P., Xu, T.-L., and Dong, X.-P. (2014) SLC17A9 protein functions as a lysosomal ATP transporter and regulates cell viability. J. Biol. Chem. 289, 23189-99

131. Zozulya, V. N., and Voloshin, I. M. (1994) Cooperative binding of quinacrine to inorganic polyphosphate. Biophys. Chem. 48, 353-358

132. Cai, X., Xu, Y., Cheung, A. K., Tomlinson, R. C., Alcázar-Román, A., Murphy, L., Billich, A., Zhang, B., Feng, Y., Klumpp, M., Rondeau, J.-M., Fazal, A. N., Wilson, C. J., Myer, V., Joberty, G., Bouwmeester, T., Labow, M. a, Finan, P. M., Porter, J. a, Ploegh, H. L., Baird, D., De Camilli, P., Tallarico, J. a, and Huang, Q. (2013) PIKfyve, a class III PI kinase, is the target of the small molecular IL-12/IL-23 inhibitor apilimod and a player in Toll-like receptor signaling. Chem. Biol. 20, 912-21

133. Freimoser, F. M., Hürlimann, H. C., Jakob, C. A., Werner, T. P., and Amrhein, N. (2006) Systematic screening of polyphosphate (poly P) levels in yeast mutant cells reveals strong interdependence with primary metabolism. Genome Biol. 7, R109

134. Indge, K. J. (1968) Polyphosphates of the yeast cell vacuole. J. Gen. Microbiol. 51, 44755

135. Neef, D. W. (2004) Novel Mechanisms for Regulating Polyphosphate. Thesis Diss.

136. Feng, J., Chen, Y., Pu, J., Yang, X., Zhang, C., Zhu, S., Zhao, Y., Yuan, Y., Yuan, H., and Liao, F. (2011) An improved malachite green assay of phosphate: mechanism and 
application. Anal. Biochem. 409, 144-9

137. Hürlimann, H. C., Stadler-Waibel, M., Werner, T. P., and Freimoser, F. M. (2007) Pho91 Is a vacuolar phosphate transporter that regulates phosphate and polyphosphate metabolism in Saccharomyces cerevisiae. Mol. Biol. Cell. 18, 4438-45

138. Bednárová, L., Palacký, J., Bauerová, V., Hrušková-Heidingsfeldová, O., Pichová, I., and Mojzeš, P. (2013) Raman microspectroscopy of the yeast vacuoles. Adv. Biomed. Spectrosc. 7, 73-77

139. Schie, I. W., and Huser, T. (2013) Methods and Applications of Raman Microspectroscopy to Single-cell Analysis. Appl. Spectrosc. 67, 993-6

140. Ikonomov, O. C., Sbrissa, D., Fligger, J., Delvecchio, K., and Shisheva, A. (2010) ArPIKfyve regulates Sac3 protein abundance and turnover: disruption of the mechanism by Sac3I41T mutation causing Charcot-Marie-Tooth 4J disorder. J. Biol. Chem. 285, $26760-4$

141. Lenk, G. M., Ferguson, C. J., Chow, C. Y., Jin, N., Jones, J. M., Grant, A. E., Zolov, S. N., Winters, J. J., Giger, R. J., Dowling, J. J., Weisman, L. S., and Meisler, M. H. (2011) Pathogenic mechanism of the FIG4 mutation responsible for Charcot-Marie-Tooth disease CMT4J. PLoS Genet. 7, e1002104 